\title{
Diagrammar in classical scalar field theory
}

\author{
E. Cattaruzza ${ }^{a}$, E. Gozzi ${ }^{a, b, 1}$, A. Francisco Neto ${ }^{c, *}$ \\ a Department of Physics (Miramare Campus), University of Trieste, Strada Costiera 11, Miramare-Grignano 34014, Trieste, Italy \\ ${ }^{\mathrm{b}}$ INFN, Sezione di Trieste, Italy \\ ${ }^{\mathrm{c}}$ Departamento de Engenharia de Produção, Administração e Economia, Escola de Minas, Campus Morro do Cruzeiro, UFOP, \\ 35400-000 Ouro Preto MG, Brazil
}

\section{A R T I C L E I N F O}

\section{Article history:}

Received 1 February 2011

Accepted 27 May 2011

Available online 13 June 2011

\section{Keywords:}

Classical field theory

Feynman diagram

\begin{abstract}
A B S T R A C T
In this paper we analyze perturbatively a $g \phi^{4}$ classical field theory with and without temperature. In order to do that, we make use of a path-integral approach developed some time ago for classical theories. It turns out that the diagrams appearing at the classical level are many more than at the quantum level due to the presence of extra auxiliary fields in the classical formalism. We shall show that a universal supersymmetry present in the classical path-integral mentioned above is responsible for the cancelation of various diagrams. The same supersymmetry allows the introduction of super-fields and super-diagrams which considerably simplify the calculations and make the classical perturbative calculations almost "identical" formally to the quantum ones. Using the super-diagrams technique, we develop the classical perturbation theory up to third order. We conclude the paper with a perturbative check of the fluctuation-dissipation theorem.
\end{abstract}

(c) 2011 Elsevier Inc. All rights reserved.

\section{Introduction}

Before starting this paper we would like to apologize with the authors of the famous paper [1] who first made use of the ancient Italian word "diagrammar" for having copied their idea. We used that same word because we feel "diagrammar" expresses in the most complete way the skills one has to develop in order to draw all possible Feynman diagrams of a classical theory which are many more

\footnotetext{
* Corresponding author.

E-mail addresses: Enrico.Cattaruzza@gmail.com (E. Cattaruzza),gozzi@ts.infn.it, ennio.gozzi@gmail.com (E. Gozzi), antfrannet@gmail.com (A. Francisco Neto).

1 Tel.: +39 0402240260; fax: +39040224601.
} 
than the quantum counterpart. We also think that the word expresses in a nice way the feeling we had when we realized that the very many classical diagrams could be encapsulated in few super-diagrams.

Let us go back to the paper. In the past fifteen years the physics of heavy ion collisions has become one of the hottest topics in high energy physics [2]. The most interesting point is that this may lead to the formation of a state of matter known as quark-gluon plasma (QGP) (for a review, see Ref. [3]). This topic has attracted the attention of many sectors of theoretical physics, last but not least, even of strings using the duality AdS/QCD (for example, see Ref. [4] and the references therein).

We know that in the first instants after the (QGP) formation, the gluon occupation number is very high [5], so one could describe the system by a classical field theory [6], analogously to what happens to QED which, when the photon occupation number is high, can be described to a good degree of accuracy by the Maxwell equations. A lot of work started in the nineties in this direction [7-10]. Most of these authors studied classical field theories by just solving the Hamilton's equations of motion and developing from there perturbative calculations. Only the author of Ref. [10] used a different route making use of a path-integral approach for classical mechanics developed in the eighties [11]. This is basically the functional counterpart of the operatorial approach to classical mechanics developed in the thirties by Koopman and von Neumann [12]. We know that the path-integral is the most natural tool to use in order to develop the perturbative calculations and the associated Feynman diagrams. It was so for the quantum path-integral (QPI) [13] and we will show that it will be the same for classical systems via the classical path-integral (CPI). In this paper, like in Refs. [7,10], we will limit ourselves to a $g \phi^{4}$ theory.

The paper is organized as follows. In Section 2 we will briefly review the CPI in order to make this paper self-contained. We shall show that, besides the phase-space variables, this formalism makes use of extra auxiliary fields. These auxiliary fields have a well-known physical meaning, being associated with the Jacobi fields [14] of the theory plus their symplectic conjugates and the response fields [15]. The presence of all these fields make the diagrammatic in the CPI rather complicated. In Section 3 we develop the formalism in the case of a field theory without temperature and present the associated diagrams which, for a point particle, have already been worked out in Ref. [16]. In Section 4 we develop the formalism in the presence of temperature and make contact with the calculations developed in Refs. $[7,10]$. We will notice that there are cancellations among various diagrams. This cancelation is due to some hidden symmetries present in the CPI. One of these is a supersymmetry and this leads to the use of super-fields (for a review see Ref. [17]) and their associated super-diagrams which we will introduce in Section 5. In the same section, we will show how the super-diagrams will allow us to reproduce the results of Section 4, but with fewer diagrams. These super-diagrams have the same vertices as in the quantum case. This is a consequence of the relation between the CPI and the QPI which was studied in Ref. [18]. In Section 6 we conclude the paper with a perturbative analysis of the fluctuation-dissipation theorem [15] using the super-diagrams developed in Section 5. We confine the detailed derivation of several results to a few Appendices.

\section{The path-integral for classical mechanics (CPI)}

In the thirties Koopman and von Neumann [12] developed an Hilbert space and operatorial approach to classical statistical mechanics. Their formalism is based on four postulates:

I. A state is given by an element $|\psi\rangle$ of an Hilbert space.

II. On this Hilbert space the operators $\hat{q}$ and $\hat{p}$ associated to the classical variables $q$ and $p$ commute, i.e. $[\hat{q}, \hat{p}]=0$. If we indicate with $\varphi^{a}=(q, p), a=1,2$, then the simultaneous diagonalization of $\hat{q}$ and $\hat{p}$ can be expressed as

$$
\hat{\varphi}^{a}\left|\varphi_{0}^{a}\right\rangle=\varphi^{a}\left|\varphi_{0}^{a}\right\rangle
$$

III. The evolution of $\psi(q, p)$ is given by the Liouville equation

$$
i \frac{\partial \psi(q, p, t)}{\partial t}=\hat{\tilde{\mathscr{H}}} \psi(q, p, t)
$$

where $\hat{\tilde{\mathscr{H}}} \equiv-i \partial_{p} H(q, p) \partial_{q}+i \partial_{q} H(q, p) \partial_{p}$ is the Hamiltonian operator. 
IV. The Liouville probability density $\rho(q, p)$ is given by

$$
\rho(q, p)=|\psi(q, p)|^{2} .
$$

As a consequence of the four postulates above also $\rho$ satisfies the Liouville equation as it should be.

As this is an operatorial formalism there should be an associated path-integral and, in fact, it is easy to build one [11]. Let us start from the classical transition amplitude $\left\langle\varphi^{a}, t \mid \varphi_{0}^{a}, t_{0}\right\rangle$ which clearly is

$$
\left\langle\varphi^{a}, t \mid \varphi_{0}^{a}, t_{0}\right\rangle=\tilde{\delta}\left[\varphi^{a}-\phi_{\mathrm{cl}}^{a}\left(t ; \varphi_{0}, t_{0}\right)\right]
$$

where $\phi_{\mathrm{cl}}$ is the solution at time $t$ of the Hamiltonian equation of motion

$$
\dot{\varphi}^{a}=\omega^{a b} \frac{\partial H}{\partial \varphi^{b}}
$$

with initial configuration $\varphi_{0}$ at time $t_{0}$. In Eq. (2.4) $\omega^{a b}$ is the symplectic matrix [19] and $H$ the Hamiltonian of the system.

Slicing the interval of time in Eq. (2.3) in $N$ small intervals and replacing the functional Dirac delta on the r.h.s of Eq. (2.3) with the expression

$$
\widetilde{\delta}\left[\varphi^{a}-\phi_{c l}^{a}\left(t ; \varphi_{0}, t_{0}\right)\right]=\widetilde{\delta}\left[\dot{\varphi}^{a}-\omega^{a b} \partial_{b} H\right] \operatorname{det}\left(\delta_{b}^{a} \partial_{t}-\omega^{a d} \partial_{d} \partial_{b} H\right),
$$

where det[ ] is a properly regularized functional determinant, we get that Eq. (2.3) can be written as

$$
\left\langle\varphi^{a}, t \mid \varphi_{0}^{a}, t_{0}\right\rangle=\int \mathscr{D}^{\prime \prime} \varphi^{a} \mathscr{D} \lambda_{a} \mathscr{D} c^{a} \mathscr{D} \overline{\mathcal{C}}_{a} \exp \left[i \int_{t_{0}}^{t} d \tau \tilde{\mathcal{L}}\right],
$$

where $\mathscr{D}^{\prime \prime} \varphi$ indicates the integration over all points, except the end points which are fixed, while the symbol $\mathscr{D}$ indicates that the end points are integrated over. The $\widetilde{\mathscr{L}}$ is

$$
\widetilde{\mathscr{L}}=\lambda_{a} \dot{\varphi}^{a}+i \bar{c}_{a} \dot{c}^{a}-\lambda_{a} \omega^{a b} \partial_{b} H-i \bar{c}_{a} \omega^{a d} \partial_{d} \partial_{b} H c^{b} .
$$

The auxiliary variables $\lambda_{a}$ have been introduced to do the Fourier transform of the Dirac delta on the r.h.s of Eq. (2.5), while the Grassmannian variables $c^{a}$ and $\bar{c}_{a}$ are needed to exponentiate the determinant in Eq. (2.5).

In the first paper of Ref. [11], formula (3.51), it was shown in a formal way that the determinant above is one. This is so even if we do a regularized calculation by discretizing the time along the lines of what has been done in Ref. [20]. This regularized calculation is going to appear in the fourth paper of Ref. [11]. What is not one or constant are the matrix elements of the matrix whose determinant we are talking above. They are in general functions of the phase-space variables. In order to obtain these matrix elements, as explained in the first paper of Ref. [11], more precisely in formulas (3.32)-(3.54) there, we should use a generating functional which contains not only the currents coupled to the phase-space variables, but also the currents coupled to the Grassmannian variables. Once these are integrated out what remains are the matrix elements mentioned above coupled, via their inverse matrix, to the Grassmannian currents. These matrix elements are physically extremely important because they give the correlations of Grassmannian variables, correlations which are related to the Lyapunov exponents of the system as explained in Ref. [21]. This is so because, as explained in the first paper of Ref. [11], the Grassmannian variables can be identified with the Jacobi fields of the system which are the variables that "connect" nearby trajectories. So this is the basic reason why we introduce the Grassmannian variables, not reproduce the determinant which is after-all just one, but to give the matrix elements which contain important physical information.

Another important issue is the regularization used in building the complete generating functional mentioned above. Usually one uses a time-slicing regularization [20] but then one has to explain [22] if the preference is for the prepoint choice or Itô's prescription (an example is given in [23]), a midpoint one, corresponding to the Stratonovich prescription, or the end-point choice. While the value of the determinant is always one, independently of the regularization prescription (and we thank one of the 
referees for pointing this out), this is not so for the matrix elements which can be different functions of phase-space. Basically, the reason is that these matrix elements are related to the effective interaction between phase-space variables and Grassmannian ones. This interaction and the associated Feynman rules change with the type of regularization prescription used (for details see Ref. [24]). The one which gives identical Feynman rules as in the continuum is the Stratonovich or midpoint one and we will stick to it.

Let us now go back to Eq. (2.6) and build the equivalent operatorial formalism. Given two variables $O_{1}$ and $O_{2}$ from the path-integral (2.6) we can derive the commutators defined as follows [13,14]

$$
\left[\hat{O}_{1}, \hat{O}_{2}\right]=\lim _{\epsilon \rightarrow 0}\left\langle O_{1}(t-\epsilon) O_{2}(t)-O_{2}(t-\epsilon) O_{1}(t)\right\rangle .
$$

The symbol \langle\rangle means the average under the path-integral and sandwiched among any state. We have put a hat ${ }^{\wedge}$ on the variables on the l.h.s. of Eq. (2.8) because they become operators via this definition. Applying (2.8) to the basic variables $\varphi^{a}, \lambda_{a}, c^{a}, \bar{c}_{a}$ we get:

$$
\left[\hat{\varphi}^{a}, \hat{\lambda}_{b}\right]=i \delta_{b}^{a}, \quad\left[\hat{c}^{a}, \hat{\bar{c}}_{b}\right]_{+}=\delta_{b}^{a},
$$

where by $[,]_{+}$we indicate the anti-commutators. All the other commutators and anti-commutators are zero in Eq. (2.9). In particular, $\left[\hat{\varphi}^{a}, \hat{\varphi}^{b}\right]=0$, which implies $[\hat{q}, \hat{p}]=0$, and this confirms that we are doing classical mechanics. From the first equation in (2.9) we get a representation for $\lambda_{a}$

$$
\hat{\lambda}_{a}=-i \frac{\partial}{\partial \varphi^{a}}
$$

and this identifies $\hat{\lambda}_{a}$ with the response field (see for example Ref. [15]).

Let us first write down the Hamiltonian associated to the Lagrangian $\tilde{\mathscr{L}}$

$$
\tilde{\mathscr{H}}=\lambda_{a} \omega^{a b} \partial_{b} H+i \bar{c}_{a} \omega^{a d} \partial_{d} \partial_{b} H c^{b} .
$$

Having obtained our operatorial formalism let us see if we can recover the KvN approach. To do that, let us consider the first piece of $\tilde{\mathscr{H}}$, which we call Bosonic one $(B)$

$$
\tilde{\mathscr{H}}_{B}=\lambda_{a} \omega^{a b} \partial_{b} H \text {. }
$$

Using Eq. (2.10) we get that $\tilde{\mathscr{H}}$ goes into the following operator

$$
\begin{aligned}
\tilde{\mathscr{H}}_{B} \rightarrow \hat{\tilde{\mathscr{H}}}_{B} & =-i \omega^{a b} \partial_{b} H \frac{\partial}{\partial \varphi^{a}} \\
& =-i \partial_{p} H \partial_{q}+i \partial_{q} H \partial_{p} \equiv \hat{L}
\end{aligned}
$$

and so we obtain the Liouville operator. This confirms that, at least for the Bosonic part, the pathintegral behind the KvN formalism is exactly the one presented in (2.6). As we said after the KvN postulates, both the elements of the KvN Hilbert space $\psi(\varphi)$ and the probability densities $\rho(\varphi)$ evolve via the same operator $\tilde{\mathcal{H}}$. As a consequence also the path-integral for the evolution of both $\psi$ and $\rho$ is the same. In this paper, as in the first papers [11], we will consider the averages taken with the probabilities densities $\rho(\varphi)$. The reason for this choice is due to the fact, as it has been proven in Ref. [25], that the Hilbert space of $\mathrm{KvN}$ is actually made of just Dirac deltas on the phase-space points: $\delta\left(\varphi-\varphi_{0}\right)=\left\langle\varphi \mid \varphi_{0}\right\rangle$, and the superposition principle does not act on these states. This is natural in CM. So saying that we use an Hilbert space formalism in CM is a rather "formal" statement, and for this reason we prefer to use the probability densities.

Going now back to $\tilde{\mathscr{H}}$ the curious reader may ask what is the mathematical meaning of $\tilde{\mathscr{H}}$ if we keep also the Grassmannian variables as in (2.11). Actually, it has been proved in Ref. [11] that it becomes a generalization of the Liouville operator known in the literature [19] as the Lie derivative of the Hamiltonian flow. This is easily proved once one realizes that the $c^{a}$ behaves under symplectic transformation as basis of differential forms of $\varphi^{a}$, while the $\bar{c}_{a}$ are the symplectic duals [19] to $c^{a}$ or, in other words, the basis of the totally anti-symmetric tensors. All the geometrical aspects of the 
path-integral for classical mechanics have been analyzed in details in [11,26]. In the second reference it has been proved that the set of variables $\varphi^{a}, c^{a}, \bar{c}_{a}, \lambda_{a}$ "coordinatize" a double-bundle over the phase-space [19]. The whole Cartan calculus $[19,26]$ can be reproduced using these variables and a set of seven charges present in this formalism [11]. Among those charges we will mention just two which are important for the rest of the paper

$$
\begin{aligned}
& \hat{Q}_{H}=i \hat{c}^{a} \hat{\lambda}_{a}-\hat{c}^{a} \partial_{a} H \\
& \hat{\bar{Q}}_{H}=i \hat{\bar{c}}_{a} \omega^{a b} \hat{\lambda}_{b}+\hat{\bar{c}}_{a} \omega^{a b} \partial_{b} H .
\end{aligned}
$$

They make up a universal $N=2$ supersymmetry for any Hamiltonian system. In fact, they close on the Hamiltonian

$$
\left[\hat{Q}_{H}, \hat{\bar{Q}}_{H}\right]=2 i \hat{\tilde{\mathscr{H}}} \text {. }
$$

We will see that, thanks to this supersymmetry, there will be many cancellations among Feynman diagrams once we embark on the perturbation theory. The geometrical meaning of these supercharges has also been investigated: they are basically the exterior derivative on the constant-energy surfaces of the phase-space. Or, in a more mathematically technical language, they are associated to the equivariant cohomology of the Hamiltonian field [27].

In this paper we will make use of another typical tool of supersymmetry which is the super-fields (for a review see Ref. [17]). This is an object which allow us to put in the same multiplet all the variables $\varphi^{a}, \lambda_{a}, c^{a}$ and $\bar{c}_{a}$. In order to do that, we have first to extend time $t$ to include two Grassmannian partners of time $\theta, \bar{\theta}$. The super-field $\Phi^{a}(t, \theta, \bar{\theta})$ is a function of $t, \theta, \bar{\theta}$ defined as

$$
\Phi^{a}(t, \theta, \bar{\theta}) \equiv \varphi^{a}+\theta c^{a}+\bar{\theta} \omega^{a b} \bar{c}_{b}+i \theta \bar{\theta} \omega^{a b} \lambda_{b} .
$$

It is then possible to give a much simpler expression for the Lagrangian in $\tilde{\mathcal{L}}(2.7)$. In fact, it is easy [18] to prove that

$$
\tilde{\mathscr{L}}=i \int d \theta d \bar{\theta} L\left[\Phi^{a}\right]+(s . t),
$$

where $L$ is the standard Lagrangian associated to the Hamiltonian $H$ of classical mechanics which we have used in the r.h.s of Eq. (2.7) and (s.t.) is a surface term.

Using the super-field it is also possible to give the following generalization of expression (2.6), for its derivation see Ref. [18],

$$
\left\langle\Phi^{a}, t \mid \Phi_{0}^{a}, t_{0}\right\rangle=\int \mathscr{D}^{\prime \prime} \Phi^{q} \mathscr{D} \Phi^{p} \exp \left\{i \int_{t_{0}}^{t} i d \tau d \theta d \bar{\theta} L[\Phi]\right\} .
$$

The indices $q$ and $p$ refer to the first and second indices $a$ of $\varphi^{a}$, respectively. They are called this way because we know that $\varphi^{1}=q$ and $\varphi^{2}=p$.

An expression similar to (2.17) can also be written for the generating functional, which instead of being the complicated object:

$$
Z[J=0]=\int \mathscr{D} \varphi^{a} \mathscr{D} \lambda_{a} \mathscr{D} c^{a} \mathscr{D} \overline{\mathcal{C}}_{a} \exp \left\{i \int_{t_{0}}^{t} d \tau \tilde{\mathcal{L}}\right\}
$$

can be written as:

$$
Z[J=0]=\int \mathscr{D} \Phi^{a} \exp \left\{i \int_{t_{0}}^{t} d \tau \int i d \theta d \bar{\theta} L[\Phi]+(\text { s.t. })\right\} .
$$

Note how similar is (2.19) to the quantum analogue:

$$
Z_{Q M}[J=0]=\int \mathscr{D} \varphi^{a} \exp \left\{\frac{i}{\hbar} \int_{t_{0}}^{t} d \tau L[\varphi]\right\} .
$$


Basically, $\varphi^{a}$ is replaced by $\Phi^{a}$ and $1 / \hbar$ by $i \int d \theta d \bar{\theta}$ while the functional weight is given in both cases by the standard Lagrangian $L$ of classical mechanics.

The similarity between Eqs. (2.19) and (2.20) has been studied in great details in Ref. [18]. In this paper this similarity will be used to drastically simplify the perturbation theory which, instead of making use of the complicated Lagrangian $\tilde{\mathcal{L}}$ of (2.7) appearing in Eq. (2.18), will make use of just the $L$ appearing in Eq. (2.19), therefore, borrowing many results from the quantum analog (2.20).

Before closing this section we would like to bring to the attention of the reader the physics behind the variables $c^{a}$. We do that because people may consider them useless. We have already made hints before to their mathematical meaning, being that of basis for differential forms (more details can be found in Ref. [26]). Their physical meaning has been explored in Ref. [21] and it goes as follows. Let us look at the equation of motion for $c^{a}$ which can be derived from the $\tilde{\mathscr{L}}$ of Eq. (2.7)

$$
\dot{c}^{a}-\omega^{a b} \frac{\partial^{2} H}{\partial \varphi^{b} \partial \varphi^{d}} c^{d}=0 .
$$

This equation is the same as the one satisfied by the first variations $\delta \varphi^{a}$ :

$$
\delta \dot{\varphi}^{a}-\omega^{a b} \frac{\partial^{2} H}{\partial \varphi^{b} \partial \varphi^{d}} \delta \varphi^{d}=0 .
$$

These first variations are also known as Jacobi fields. (See for example Ref. [14].) So we can identify $c^{a} \approx \delta \varphi^{a}$. The correlations of the Jacobi fields are related to the Lyapunov exponents of chaotic systems:

$$
\lim _{t \rightarrow \infty}\langle\delta \varphi(0) \delta \varphi(t)\rangle \sim e^{-\lambda t}
$$

where $(\lambda)$ is the highest Lyapunov exponent of the system. This is equivalent to saying that the correlations of $c^{a}, \bar{c}_{a}$ gives the Lyapunov exponents [21]. So, we see that the $c^{a}, \bar{c}_{a}$ are crucial ingredients to get a full information on the dynamical system [21] we are studying. We talk about correlations between $c^{a}$ and $\bar{c}_{a}$ because the correlations among the $c^{a}$ only give zero (see Refs. [28,21, 29]). The $\bar{c}_{a}$ are the symplectic duals of $c^{a}$ and, once they are multiplied by the symplectic matrix, they can also be identified with the Jacobi fields. So the correlations among $c^{a}, \bar{c}_{a}$ are also equivalent to correlations among Jacobi fields. Of course, the Lyapunov exponents could be obtained in many other ways [30] without using the $c^{a}, \bar{c}_{a}$, but we find this manner rather elegant. Actually, we know that classical mechanics could be built by just using the $\varphi^{a}$, and so the variables $\lambda_{a}, c^{a}$ and $\bar{c}_{a}$ are redundant variables and this redundancy is signaled by the symmetries present in $\tilde{\mathcal{L}}$ like the supersymmetry and other invariances. But, as usual in physics, the redundancies and the symmetries make things more elegant and allows the use of all the tools that group theory put at our disposal. In our case, if we had not used all the variables, we would not be able to build the super-field and use the expression (2.19) for the perturbation theory.

Before starting next section we should introduce for completeness a new representation of the commutator algebra (2.9). More details can be found in Ref. [18]. This new representation will be very important in order to do the perturbation theory using super-fields. We had seen that $\tilde{\mathcal{L}}$ in Eq. (2.16) and $L[\Phi]$ differ by a surface term (s.t) and the same will happen at the level of generating functionals (2.17) and (2.19). In order to get rid of that surface term the trick [18] is to change the representation in (2.10) that we derived from the commutator (2.9). We could, for example, represent $\hat{q}$ and $\hat{\lambda}_{p}$ as multiplicative operators and $\hat{p}$ and $\hat{\lambda}_{q}$ as derivative ones

$$
\begin{aligned}
& \hat{p}=i \frac{\partial}{\partial \lambda_{p}} \\
& \hat{\lambda}_{q}=-i \frac{\partial}{\partial q} .
\end{aligned}
$$

Analogously, we can proceed for the Grassmannian variables: represent $\hat{\bar{c}}^{q}$ and $\hat{\bar{c}}_{p}$ as multiplicative operators and $\hat{c}^{p}$ and $\hat{\bar{c}}_{q}$ as derivative ones: 


$$
\begin{aligned}
& \hat{c}^{p}=\frac{\partial}{\partial \bar{c}_{p}} \\
& \hat{\bar{c}}_{q}=\frac{\partial}{\partial c^{q}} .
\end{aligned}
$$

The generalized states [18] in the extended Hilbert space, including the forms, will then be $\mid q, \lambda_{p}$, $\left.c^{q}, \bar{c}_{p}\right\rangle$. Note that the variables $q, \lambda_{p}, c^{q}, \bar{c}_{p}$ are exactly those which enter the super-field $\Phi^{q}$

$$
\Phi^{q}=q+\theta c^{q}+\bar{\theta} \bar{c}_{p}+i \theta \bar{\theta} \lambda_{p}
$$

This is crucial in order to give a complete super-field representation of our theory without any surface terms. We invite the reader to master the details contained in Ref. [18] before embarking in the rest of this paper.

\section{Perturbation theory without temperature}

What we will do in this section for a $g \phi^{4}$ scalar field theory, has been done partly for a point particle in Ref. [16]. We use the word "partly" because those authors neglected the Grassmannian variables $c^{a}$ and $\bar{c}_{a}$. So their analysis apply only to the zero-form sector of the theory. We called this sector this way because, as indicated before, the $c^{a}$ are the basis of the differential forms $[19,26]$.

Let us start with a scalar field theory whose Hamiltonian is

$$
H=\int d^{4} x\left[\frac{\pi^{2}}{2}+\frac{(\nabla \phi)^{2}}{2}+\frac{m^{2} \phi^{2}}{2}+g \frac{\phi^{4}}{4 !}\right]
$$

where $\pi(x)$ is the momentum conjugate to the field $\phi(x)$. We will indicate with $\mathscr{H}$ the Hamiltonian density:

$$
\mathscr{H}(x)=\frac{\pi^{2}(x)}{2}+\frac{[\nabla \phi(x)]^{2}}{2}+\frac{m^{2} \phi^{2}(x)}{2}+g \frac{\phi^{4}(x)}{4 !} .
$$

Let us next build the generating functional associated to the classical path-integral of the previous section, but we shall choose only those trajectories that start from a fixed point in phase-space $\varphi_{i}=\left(\phi_{i}, \pi_{i}\right)$ and we will not average over this initial configuration. The expression is:

$$
\begin{aligned}
& \mathcal{Z}_{\varphi_{i}}\left[J_{\phi}, J_{\lambda^{\pi}}, \bar{J}_{C^{\phi}}, J_{\bar{c}_{\pi}}\right] \\
& \equiv \int \mathscr{D}^{\prime} \varphi \mathscr{D} \lambda \mathscr{D} \boldsymbol{C} \mathscr{D} \bar{c} \exp \left\{i \tilde{\mathscr{S}}+i \int_{t_{i}}^{t_{f}} d t \int d^{3} \vec{x}\left[J_{\phi} \phi+J_{\lambda_{\pi}} \lambda_{\pi}-i \bar{j}_{C} \phi C^{\phi}-i \bar{c}_{\pi} J_{\bar{c}_{\pi}}\right]\right\}
\end{aligned}
$$

where

$$
\tilde{\vartheta}=\int_{t_{i}}^{t_{f}} d t \int d^{3} \vec{x}\left(\lambda_{a} \dot{\varphi}^{a}+i \bar{c}_{a} \dot{c}^{a}-\lambda_{a} \omega^{a b} \partial_{b} H-i \bar{c}_{a} \omega^{a d} \partial_{d} \partial_{b} H c^{b}\right)
$$

with the $H$ given by Eq. (3.1) and the symbol $\mathscr{D}^{\prime}$ indicates the "sum" over all the trajectories starting from a fixed $\varphi_{i}$ and not integrating over the initial configuration. In the $\lambda, c, \bar{c}$ we have put as indices $\phi, \pi$ to indicate either the first or the second set of indices " $a$ " as in postulate II of Section 2. To the currents we have coupled only the "configuration variables" in the representation (2.25) where $\phi, \lambda_{\pi}, c^{\phi}, \bar{c}_{\pi}$ are multiplicative operators. We did that because, as it is clear from (2.25), it will be straightforward to pass to the super-field formulation. The peculiar combination of " \pm " and " $i$ " in the coupling of current and fields (3.3) is also related to the fact that eventually we also want to build a super-current to couple it to the super-field.

As it is usually done, let us divide $\tilde{s}$ into a free part:

$$
\tilde{s}_{0} \equiv \int_{t_{i}}^{t_{f}} d t \int d^{3} \vec{x}\left[\lambda_{a} \dot{\phi}^{a}-\lambda_{\phi} \pi-\lambda_{\pi}\left(\nabla^{2}-m^{2}\right) \phi+i \bar{c}_{a} \dot{c}^{a}-i \bar{c}_{\phi} c^{\pi}-i \bar{c}_{\pi}\left(\nabla^{2}-m^{2}\right) c^{\phi}\right]
$$


and an interaction one:

$$
\tilde{s}_{V} \equiv \int d^{4} x\left(\frac{g}{3 !} \lambda_{\pi} \phi^{3}+\frac{i g}{2 !} \bar{c}_{\pi} \phi^{2} c^{\phi}\right),
$$

where we indicate with $\int d^{4} x$ the expression $\int_{t_{i}}^{t_{f}} d t \int d^{3} \vec{x}$. To develop the perturbation theory, as it is usually done in quantum field theory, in the $\tilde{s}_{V}$ of $\mathcal{Z}$ we shall replace the fields with derivative operators with respect to the associated currents as follows:

$$
\phi \rightarrow \frac{1}{i} \frac{\delta}{\delta J_{\phi}}, \lambda_{\pi} \rightarrow \frac{1}{i} \frac{\delta}{\delta J_{\lambda_{\pi}}}, c^{\phi} \rightarrow \frac{\delta}{\delta \bar{J}_{c^{\phi}}}, \bar{c}_{\pi} \rightarrow-\frac{\delta}{\delta J_{\bar{c}_{\pi}}}
$$

so we get:

$$
\tilde{s}_{V}\left[\frac{1}{i} \frac{\delta}{\delta J_{\phi}}, \frac{1}{i} \frac{\delta}{\delta J_{\phi}}, \frac{\delta}{\delta \bar{J}_{C^{\phi}}},-\frac{\delta}{\delta J_{\bar{c}_{\pi}}}\right] \equiv \int d^{4} x\left(\frac{g}{3 !} \frac{\delta}{\delta J_{\lambda_{\pi}}} \frac{\delta^{3}}{\delta J_{\phi}^{3}}+\frac{i g}{2 !} \frac{\delta}{\delta J_{\bar{c}_{\pi}}} \frac{\delta^{2}}{\delta J_{\phi}^{2}} \frac{\delta}{\delta \bar{J}_{c^{\phi}}}\right) .
$$

It is then easy to write $\mathcal{Z}_{\varphi_{i}}\left[J_{\phi}, J_{\lambda^{\pi}}, \bar{J}_{c^{\phi}}, J_{\bar{c}_{\pi}}\right]$ in Eq. (3.3) as follows:

$$
\mathcal{Z}_{\varphi_{i}}\left[J_{\phi}, J_{\lambda_{\pi}}, \bar{J}_{c}, J_{\bar{c}_{\pi}}\right]=\exp \left\{i \tilde{\vartheta}_{V}\left[\frac{1}{i} \frac{\delta}{\delta J_{\phi}}, \frac{1}{i} \frac{\delta}{\delta J_{\phi}}, \frac{\delta}{\delta \bar{J}_{c^{\phi}}},-\frac{\delta}{\delta J_{\bar{c}_{\pi}}}\right]\right\} \mathcal{Z}_{\varphi_{i}}^{(0)}\left[J_{\phi}, J_{\lambda_{\pi}}, \bar{J}_{c^{\phi}}, J_{\bar{c}_{\pi}}\right]
$$

where $\mathcal{Z}_{\varphi_{i}}^{(0)}$ is the free generating functional built out of the $\tilde{s}_{0}$. It is easy to prove that this part $\mathcal{Z}_{\varphi_{i}}^{(0)}$ can be factorized into a "Bosonic" (B) part for the variables $\varphi$ and $\lambda$ and a "Fermionic" one (F) for the Grassmannian variables $c, \bar{c}$ :

$$
\mathcal{Z}_{\varphi_{i}}^{(0)}\left[J_{\phi}, J_{\lambda_{\pi}}, \bar{J}_{c}, J_{\bar{c}_{\pi}}\right]=\mathcal{Z}_{(B)}^{(0)}\left[J_{i}, J_{\lambda_{\pi}}\right] \mathfrak{Z}_{(F)}^{(0)}\left[\bar{J}_{C} \phi, J_{\bar{c}_{\pi}}\right]
$$

where

$$
\begin{aligned}
& \mathcal{Z}_{(B) \varphi_{i}}^{(0)}\left[J_{\phi}, J_{\lambda_{\pi}}\right] \\
& \quad=\int \mathscr{D}^{\prime} \varphi \mathscr{D} \lambda \exp \left\{i \int_{t_{i}}^{t_{f}} d t \int d^{3} \vec{x}\left[\lambda_{a} \dot{\phi}^{a}-\lambda_{\phi} \pi-\lambda_{\pi}\left(\nabla^{2}-m^{2}\right) \phi+J_{\phi} \phi+J_{\lambda_{\pi}} \lambda_{\pi}\right]\right\}
\end{aligned}
$$

and

$$
\begin{aligned}
\mathcal{Z}_{(F)}^{(0)}\left[\bar{J}_{c^{\phi}}, J_{\bar{c}_{\pi}}\right]= & \int \mathscr{D C} \mathscr{D} \bar{c} \exp \left\{\int _ { t _ { i } } ^ { t _ { f } } d t \int d ^ { 3 } \vec { x } \left[i \bar{c}_{a} \dot{c}^{a}-i \bar{c}_{\phi} c^{\pi}\right.\right. \\
& \left.\left.-i \bar{c}_{\pi}\left(\nabla^{2}-m^{2}\right) c^{\phi}-i \bar{J}_{c^{\phi}} c^{\phi}-i \bar{c}_{\pi} J_{\bar{c}_{\pi}}\right]\right\} .
\end{aligned}
$$

All the fields enter at most in a quadratic form. It is easy to integrate them out in Eq. (3.11) as it has been done in Ref. [16]. The result is:

$$
\mathcal{Z}_{(B) \varphi_{i}}^{(0)}\left[J_{\phi}, J_{\lambda_{\pi}}\right]=\exp \left[i \int d^{4} x J_{\phi}(x) \phi_{0}(x)+i \int d^{4} x d^{4} x^{\prime} J_{\phi}(x) G_{R}\left(x-x^{\prime}\right) J_{\lambda_{\pi}}\left(x^{\prime}\right)\right]
$$

where $\phi_{0}(x)$ is a solution to the equation

$$
\left(\square+m^{2}\right) \phi=0
$$

and $G_{R}$ is the retarded propagator satisfying

$$
\left(\square+m^{2}\right) G_{R}=-\delta(x) .
$$

Its expression is:

$$
G_{R}(x)=\int \frac{d^{4} p}{(2 \pi)^{4}} \frac{e^{-i p \cdot x}}{\left(p^{0}+i \epsilon\right)^{2}-\vec{p}^{2}-m^{2}} .
$$


Also for the $\mathcal{Z}_{(F)}^{(0)}$ we can easily integrate out the fields (for details see Ref. [31]) and obtain:

$$
\mathcal{Z}_{(F)}^{(0)}\left[\bar{J}_{C^{\phi}}, J_{\bar{c}_{\pi}}\right]=\exp \left[-\int d^{4} x d^{4} x^{\prime} \bar{J}_{C^{\phi}}(x) G_{R}\left(x-x^{\prime}\right) J_{\bar{c}_{\pi}}\left(x^{\prime}\right)\right] .
$$

We have now all the tools to obtain all the propagators.

Let us start at the zero order in perturbation theory and we will draw next to them the diagrams:

$$
\begin{aligned}
& \langle\phi(x)\rangle^{0}=\left.\frac{1}{i} \frac{\delta}{\delta J_{\phi}} \mathcal{Z}_{\varphi_{i}}^{(0)}\left[J_{\phi}, J_{\lambda_{\pi}}, \bar{J}_{c}, J_{\bar{c}_{\pi}}\right]\right|_{J_{\phi}, J_{\lambda_{\pi}}, \bar{J}_{C}, J_{\bar{c}_{\pi}}=0} \\
& =\left.\frac{1}{i} \frac{\delta}{\delta J_{\phi}} \mathcal{Z}_{B \varphi_{i}}^{(0)}\left[J_{\phi}, J_{\lambda_{\pi}}\right]\right|_{J_{\phi}, J_{\lambda_{\pi}}=0} \\
& =\phi_{0}(x) \equiv \underset{x}{\bullet-} \\
& \left\langle\phi\left(x_{2}\right) \phi\left(x_{1}\right)\right\rangle^{0}=\phi_{0}\left(x_{2}\right) \phi_{0}\left(x_{1}\right) \equiv \underset{x_{1}}{\bullet} \longrightarrow x_{2} \\
& \left\langle\lambda_{\pi}\left(x_{2}\right) \phi\left(x_{1}\right)\right\rangle^{0}=\left.\left(\frac{1}{i}\right)^{2} \frac{\delta}{\delta J_{\lambda_{\pi}}\left(x_{2}\right)} \frac{\delta}{\delta J_{\phi}\left(x_{1}\right)} Z_{\varphi_{i}}^{(0)}\left[J_{\phi}, J_{\lambda_{\pi}}, \bar{J}_{c}, J_{\bar{c}_{\pi}}\right]\right|_{J_{\phi}, J_{\lambda_{\pi}}, \bar{J}_{c} \phi, \bar{c}_{\bar{c}_{\pi}}=0} \\
& =-i G_{R}\left(x_{1}-x_{2}\right)=G_{\lambda_{\pi} \phi} \equiv \bullet--\longrightarrow x_{2}
\end{aligned}
$$

In Eq. (3.20) we borrow from Ref. [7]. the notation of full-dashed propagator. In Eq. (3.19) we have drawn a diagram made of two pieces not linked to each other, because they are actually the product of two separated fields and not a propagator. We will have them soldered to each other once we use the thermal average over the initial conditions.

Analogously to Eq. (3.20) we can calculate

$$
\left\langle\phi\left(x_{2}\right) \lambda_{\pi}\left(x_{1}\right)\right\rangle^{0}=-i G_{R}\left(x_{2}-x_{1}\right)=G_{\phi \lambda_{\pi}} \equiv \underset{x_{1}}{\bullet-\bullet} x_{2}
$$

The correlations among Grassmannian variables give:

$$
\begin{aligned}
& \left\langle\bar{c}_{\pi}\left(x_{2}\right) c^{\phi}\left(x_{1}\right)\right\rangle^{0}=-\left.\frac{\delta}{\delta J_{\bar{c}_{\pi}}\left(x_{2}\right)} \frac{\delta}{\delta \bar{J}_{c^{\phi}}\left(x_{1}\right)} \mathcal{Z}_{F}^{(0)}\left[\bar{J}_{c^{\phi}}, J_{\bar{c}_{\pi}}\right]\right|_{\bar{J}_{c}, J_{\bar{c}_{\pi}}=0}
\end{aligned}
$$

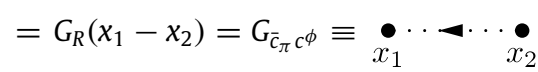

and

$$
\begin{aligned}
& \left\langle c^{\phi}\left(x_{2}\right) \bar{c}_{\pi}\left(x_{1}\right)\right\rangle^{0}=\left.\frac{\delta}{\delta \bar{J}_{c}\left(x_{2}\right)}\left[-\frac{\delta}{\delta J_{\bar{c}_{\pi}}\left(x_{1}\right)}\right] \mathcal{Z}_{F}^{(0)}\left[\bar{J}_{c}, J_{\bar{c}_{\pi}}\right]\right|_{\bar{J}_{c} \phi, J \bar{J}_{\pi}}=0
\end{aligned}
$$

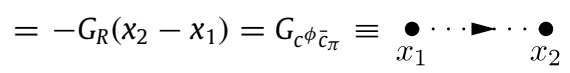

Above we have adopted the convention of putting an arrow which points from $c$ to $\bar{c}$ (see Ref. [31]).

As both the propagators, of the $\phi \phi$ and $c \bar{c}$, are related to the $G_{R}$ we will sometimes put an index "(F)" or "(B)" to indicate if it comes from the Bosonic fields or "Fermionic" (Grassmannian) ones. The fact that the two propagators are equal (modulo $i$ ) to each other is due to the supersymmetry present in this formalism [11]. We will see other manifestation of it later on in several cancellations among diagrams.

Let us now derive the rule for the vertices. Expanding $e^{i \tilde{\delta}_{V}}$ in Eq. (3.9) to the first order in $g$, we get:

$$
i \tilde{\delta}_{V}\left[\frac{1}{i} \frac{\delta}{\delta J_{\phi}}, \frac{1}{i} \frac{\delta}{\delta J_{\lambda_{\pi}}}, \frac{\delta}{\delta \bar{J}_{c^{\phi}}},-\frac{\delta}{\delta J_{\bar{c}_{\pi}}}\right] \equiv \int d^{4} x\left[i g\left(\frac{1}{3 !} \frac{\delta}{\delta J_{\lambda_{\pi}}} \frac{\delta^{3}}{\delta J_{\phi}^{3}}\right)-g\left(\frac{1}{2 !} \frac{\delta}{\delta J_{\bar{c}_{\pi}}} \frac{\delta^{2}}{\delta J_{\phi}^{2}} \frac{\delta}{\delta \bar{J}_{C} \phi}\right)\right] .
$$

Keeping account of the symmetry factors we get the following rules for the vertices:

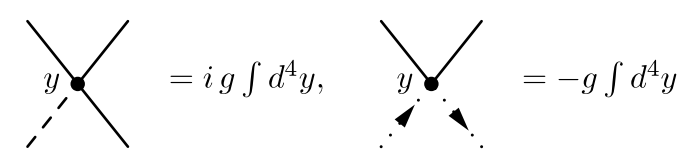


where the continuous line refers to the field $\phi$, the dashed one to the field $\lambda$ and the dotted one to $c$ or $\bar{c}$.

Let us calculate the first order correction to the expectation value of the field $\left\langle\phi_{0}(x)\right\rangle$, i.e. $\left\langle\phi_{0}(x)\right\rangle$ (the super-index (1) on $\langle\phi\rangle$ indicates the first order correction). Using the vertices and the diagrams we get (where $y$ is integrated over)

$$
\langle\phi(x)\rangle_{\varphi_{i}}^{(1)}=\left[\frac{1}{2 !} \bullet \frac{1}{x} \frac{1}{y}+\frac{1}{3 !} \bullet-\frac{1}{2 !} \underset{y}{\bullet} \frac{\vdots}{\vdots}\right.
$$

The analytic expression of the first diagram above is

$$
\underset{x}{\bullet} \frac{\vdots}{1} \underbrace{}_{y}=i g \int d^{4} y\left[\phi_{0}^{2}(y) G_{\lambda_{\pi} \phi}(0) \phi_{0}(x)\right]
$$

while the expression for the third one is

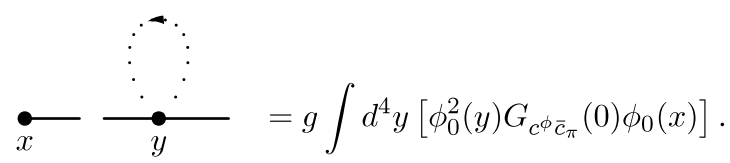

Let us remember from (3.21) and (3.23) that

$$
G_{c} \bar{c}_{\pi}=-G_{R}=-i G_{\lambda_{\pi} \phi}
$$

so (3.27) and (3.28) cancel each other and the only correction left in (3.26) is

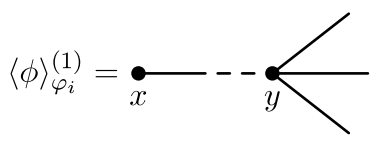

whose analytic expression is

$$
\text { : }
$$

The reader could, at this point, claim that the loops of the retarded propagators appearing in Eq. (3.26) are both zero, because of the $\theta(t)$ which appears in all retarded propagators. For a loop to be zero we have anyhow to assume that $\theta(0)=0$. This regularization of the $\theta$ corresponds to a prepoint choice in a discretized form of $t$ [22]. The prepoint choice has some problems once it is used in the path-integral. It has in fact been proved in Ref. [24] that only if one uses the midpoint choice then the Feynman rules which can be read off the continuum version are the same as the one of the discretized version. So, for this reason, we have preferred the midpoint discretization which gives $\theta(0)=1 / 2$ and this implies that the retarded loops are not zero.

Going now back to our diagrams the reader may be puzzled by diagrams like (3.30) where there are just three lines at the end going nowhere or like the single line in (3.18) also going nowhere. This is due to the fact that our generating functional $\mathcal{Z}_{\varphi_{i}}$ depends on the initial configuration which is not averaged over. These kind of diagrams, but without Grassmannian variables, were first developed in [16] and are somehow similar to those developed for fluid dynamics in [32]. Perturbation theory and something similar to Feynman diagrams where also developed long ago in [33]. We have not tried to compare our formalism with this other one. For sure our formalism is different from these other ones because it contains extra ingredients like the Grassmannian variables. Moreover, because 
of these extra ingredients, we will be able to develop the super-diagrams which will simplify the diagrammatics.

We will continue now to the second order for the $\langle\phi\rangle$. The result that can easily be obtained (where $y_{1}$ and $y_{2}$ are integrated over) is the following:

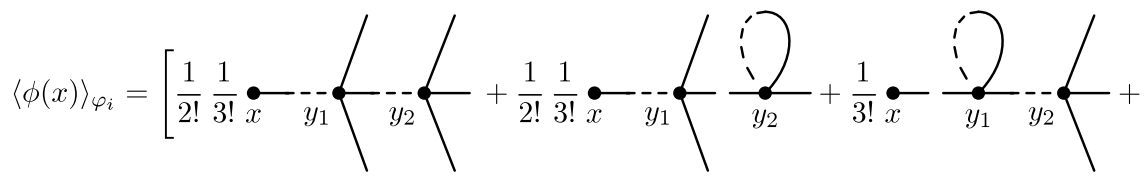

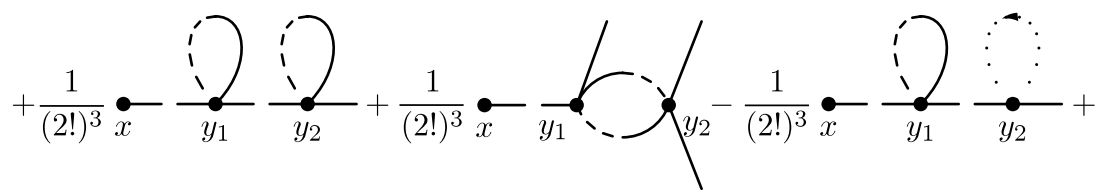

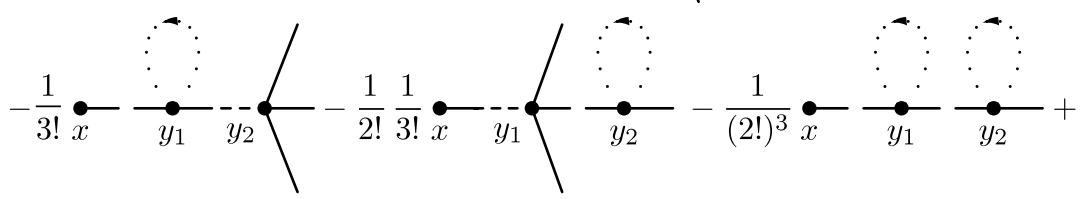

$$
\begin{aligned}
& -\frac{1}{(2 !)^{3}} \stackrel{-}{x} \underset{y_{1}}{-} \cdot\left\{y_{2}\right]
\end{aligned}
$$

As before, it is easy to see that there are cancellations. The second diagram is canceled by the eighth one, the third by the seventh, the fourth is canceled by the sum of the sixth and ninth, the fifth one by the tenth. So, the final result is

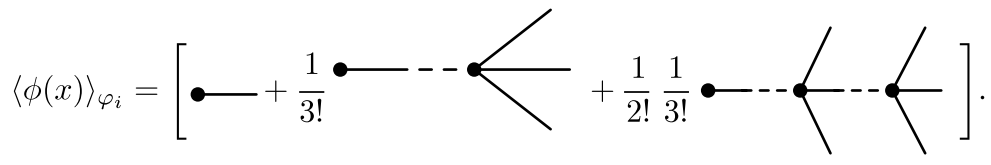

Note that no loop is left in the previous equation.

The various symmetry factors have being obtained by working out the analytical details from the $\mathcal{Z}_{\varphi_{i}}$ in (3.9) up to second order.

Let us now go back to the two-point function which, to zero order, was given in Eq. (3.19). After some tedious calculations with the second derivative of $\mathcal{Z}_{\varphi_{i}}[J]$, we get:

$$
\begin{aligned}
& \left\langle\phi\left(x_{2}\right) \phi\left(x_{1}\right)\right\rangle_{\varphi_{i}}^{(1)}=\left[\frac{1}{2 !} \underset{x_{1}}{\bullet} \stackrel{\vdots}{\vdots} \underset{x_{2}}{\bullet}+\frac{1}{3 !} \underset{x_{1}}{\bullet}-\right. \\
& \left.+\frac{1}{3 !} \underset{x_{1}}{\longrightarrow} \longrightarrow-\frac{1}{x_{2}} \underset{2 !}{\bullet} \stackrel{\vdots}{x_{1}} \stackrel{\vdots}{\bullet} \underset{x_{2}}{\bullet}\right]
\end{aligned}
$$

The first and the fourth diagrams cancel each other and, so, the final result is

$$
\left\langle\phi\left(x_{2}\right) \phi\left(x_{1}\right)\right\rangle_{\varphi_{i}}^{(1)}=\left[\frac{1}{3 !} \underset{x_{1}}{\bullet} \longrightarrow\right.
$$


Going to the second order, after some long but straightforward calculations, we obtain:

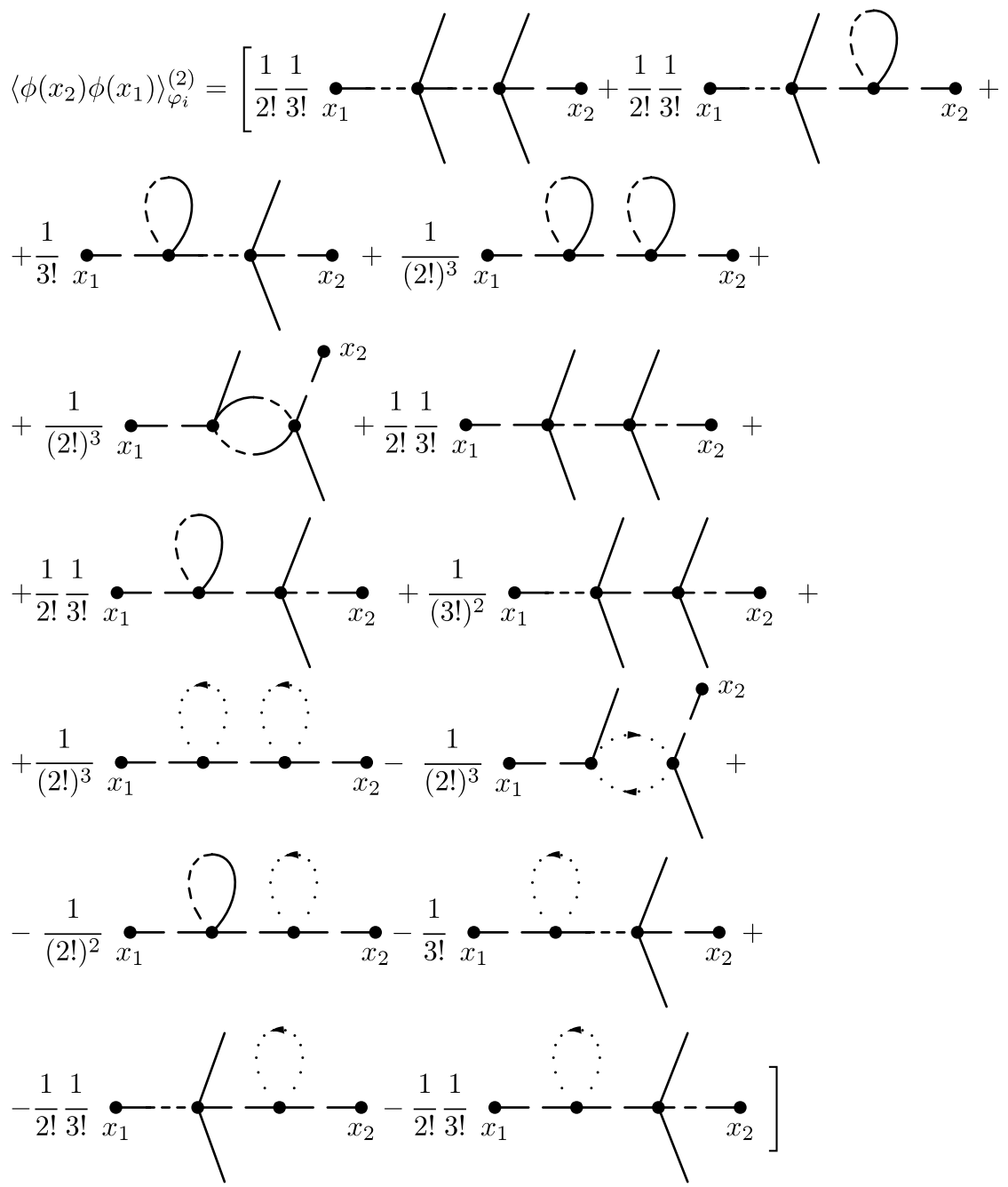

There are the usual cancellations between various diagrams and the final result is:

$$
\left\langle\phi\left(x_{2}\right) \phi\left(x_{1}\right)\right\rangle_{\varphi_{i}}^{(2)}=\left[\frac{1}{2 !} \frac{1}{3 !} \bullet-\phi-\frac{1}{x_{1}}+\frac{1}{2 !} \bullet\right.
$$

Note that also here no loop is left. This is the real sign that we are doing a classical perturbation theory. When we introduce the temperature later on loops will appear, but they are due to temperature and not quantum effects. Actually, loops would appear any time we do an average over the initial 
conditions so we cannot strictly say that no loops is a sign of classicality, we can say that no loop is the sign of a generating functional which has no average over the initial configurations.

Collecting now all diagrams developed before for the two-point functions, up to second order, we get:

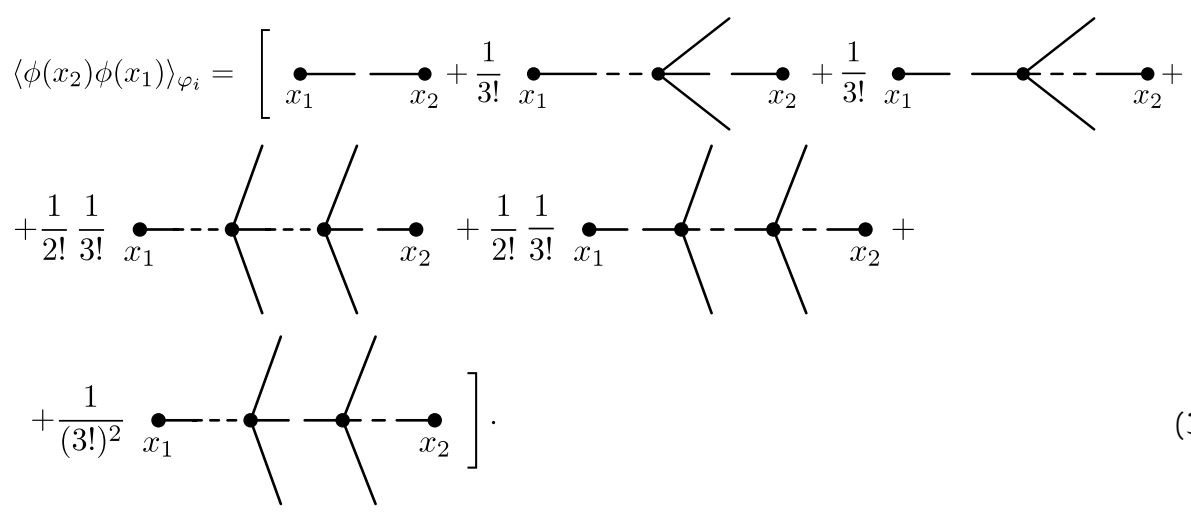

The rules for the symmetry factors are the following:

(1) put a factor of $1 / n$ ! where $n$ is the number of "free" legs, i.e. those not starting or ending at $x_{1}$ or $x_{2}$

(2) add a factor of $1 / 2$ ! for any exchange of propagators which would leave the diagram invariant.

Before concluding this section we would like to answer two questions that for sure many readers may have. The first question is why we could not proceed like in standard quantum tree-level perturbation theory. The reason is that in the classical case (in contrast to the quantum case) the initial average is made of both: the field $\phi$ and its conjugate momentum $\pi$. Moreover in the quantum case we would obtain, in doing the semi-classical limit, a van Vleck determinant. The second question, that we feel is somehow related to the first one, is why all the diagrams in this section are disconnected or have legs going nowhere. The reason is not related to the fact that we may have used the wrong generating functional, but is due to the presence of the diagram (3.18). To convince the reader, let us give the analytical expression for some of the most "strange" diagrams. For example, the 10th diagram in (3.36) where there are both disconnected pieces and lines going nowhere:

$$
\begin{aligned}
x_{1} x_{1} & {\left[\int y_{2} d^{4} y_{1} d^{4} y_{2} \phi_{0}\left(y_{1}\right)^{2} \phi_{0}\left(y_{2}\right)^{2} G_{R}^{(F)}\left(x_{1}-x_{2}\right) G_{R}^{(F)}\left(x_{2}-x_{1}\right)\right] } \\
& \times \phi_{0}\left(x_{1}\right) \phi_{0}\left(x_{2}\right) .
\end{aligned}
$$

We see that we have at $x_{1}$ and $x_{2}$ the fields $\phi_{0}\left(x_{1}\right)$ and $\phi_{0}\left(x_{2}\right)$ and their diagram is the one of (3.18). In $y_{1}$ and $y_{2}$ we also have two lines going nowhere and, according to (3.18) there must be two fields $\phi_{0}(y)$. The rest, between $y_{1}$ and $y_{2}$, are two "Fermionic" retarded propagators.

The reason we cannot connect $x_{1}$ with $y_{1}$ or $y_{2}$ with $x_{2}$ with a continuous line is because we do not have this kind of propagator in the formalism with fixed initial condition. The only propagators we have are those in (3.20)-(3.23), which are the dash-full propagator or the Fermionic dot propagators. For the $\phi \phi$ we have only the disconnected propagators (3.19). The reader may think that by coupling in some way various dash-full propagators one could get a $\phi \phi$ propagator. If one tries, it is easy to convince himself that this is not possible.

In this section, we have studied the "average" of a single field $\phi$ or correlations of two fields $\phi \phi$. It is possible to do the same for the $\lambda_{\pi}$ fields and the $c^{\phi}, \bar{c}_{\pi}$ ones. Instead of doing them explicitly we will work out the super-field perturbation theory for $\mathcal{Z}_{\varphi_{i}}[J]$ in Appendix A. We can then project out the components of the super-fields and get all the correlations mentioned above. We advise the reader not to jump immediately to Appendix A, but to wait till he has read Section 4. 


\section{Perturbation theory with temperature}

Let us now suppose that, instead of working with some fixed initial configuration, as in Section 3 , we do a thermal average over the initial configuration. Let us use the following notation: $\vec{x}$ for the three-dimensional vector and $x$ for the four-dimensional one. Let us remember that in Section 3 we choose a solution $\phi_{0}(\vec{x}, t)$ of the Klein-Gordon equation:

$$
\left(\partial_{t}^{2}-\nabla^{2}+m^{2}\right) \phi_{0}(\vec{x}, t)=0 .
$$

Its Fourier transform $\tilde{\phi}_{0}(\vec{p}, t)$ is:

$$
\phi_{0}(\vec{x}, t)=\int \frac{d^{3} \vec{p}}{(2 \pi)^{3}} \tilde{\phi}_{0}(\vec{p}, t) e^{i \vec{p} \cdot \vec{x}}
$$

and satisfies the equation:

$$
\left(\partial_{t}^{2}-E_{\vec{p}}^{2}\right) \tilde{\phi}_{0}(\vec{p}, t)=0
$$

whose solution has the form

$$
\tilde{\phi}_{0}(\vec{p}, t)=\phi_{i}(\vec{p}) \cos \left[E_{\vec{p}}\left(t-t_{i}\right)\right]+\frac{\pi_{i}(\vec{p})}{E_{\vec{p}}} \sin \left[E_{\vec{p}}\left(t-t_{i}\right)\right]
$$

with $t_{i}, \phi_{i}, \pi_{i}$ the initial time and field configurations. So the final expression for the field is:

$$
\phi_{0}(\vec{x}, t)=\int \frac{d^{3} \vec{p}}{(2 \pi)^{3}}\left\{\phi_{i}(\vec{p}) \cos \left[E_{\vec{p}}\left(t-t_{i}\right)\right]+\frac{\pi_{i}(\vec{p})}{E_{\vec{p}}} \sin \left[E_{\vec{p}}\left(t-t_{i}\right)\right] e^{i \vec{p} \cdot \vec{x}}\right\} .
$$

The thermal-averaged correlation functions that we want to evaluate are defined, for example, for the correlation involving two fields $\phi$, as follows:

$$
\langle\phi \phi\rangle_{\beta}=\frac{\int \mathscr{D} \phi_{i}(\vec{x}) \mathscr{D} \pi_{i}(\vec{x})\langle\phi \phi\rangle_{\varphi_{i} \equiv\left(\phi_{i}, \pi_{i}\right)} e^{-\beta H\left(\phi_{i}, \pi_{i}\right)}}{\int \mathscr{D} \phi_{i}(\vec{x}) \mathscr{D} \pi_{i}(\vec{x}) e^{-\beta H\left(\phi_{i}, \pi_{i}\right)}}
$$

where $\phi_{i}(\vec{x}), \pi_{i}(\vec{x})$ are the anti-Fourier transforms of the $\phi_{i}(\vec{p})$ and $\pi(\vec{p})$ appearing in Eq. (4.4).

On the correlation $\langle\phi \phi\rangle_{\beta}$ we have not indicated the argument of the fields. We did that in order to simplify the notation for the moment.

The analog of the generating functional (3.9) will now be:

$$
\mathcal{Z}_{\beta}\left[J_{\phi}\right]=\frac{\int \mathscr{D} \phi_{i}(\vec{x}) \mathscr{D} \pi_{i}(\vec{x}) e^{-\beta H\left(\phi_{i}, \pi_{i}\right)} \mathcal{Z}_{\phi_{i}}[J]}{\int \mathscr{D} \phi_{i}(\vec{x}) \mathscr{D} \pi_{i}(\vec{x}) e^{-\beta H\left(\phi_{i}, \pi_{i}\right)}} .
$$

In order to do the integration over the initial configuration in (4.7), let us first do the anti-Fourier transform of the $\phi(\vec{p})$ and $\pi(\vec{p})$ :

$$
\begin{aligned}
\phi_{i}(\vec{p}) & =\int d^{3} \vec{x} e^{-i \vec{p} \cdot \vec{x}} \phi_{i}(\vec{x}) \\
\pi_{i}(\vec{p}) & =\int d^{3} \vec{x} e^{-i \vec{p} \cdot \vec{x}} \pi_{i}(\vec{x}) .
\end{aligned}
$$

So we can write $\phi_{0}(\vec{x}, t)$ as:

$$
\phi_{0}(\vec{x}, t)=\int d^{3} \vec{x}^{\prime} \phi_{i}\left(\vec{x}^{\prime}\right) a_{\phi}\left(\vec{x}-\vec{x}^{\prime}, t\right)+\int d^{3} \vec{x}^{\prime} \pi_{i}\left(\vec{x}^{\prime}\right) a_{\pi}\left(\vec{x}-\vec{x}^{\prime}, t\right)
$$

where

$$
\begin{aligned}
& a_{\phi}\left(\vec{x}-\vec{x}^{\prime}, t\right)=\int d^{3} \vec{x} e^{-i \vec{p} \cdot\left(\vec{x}-\vec{x}^{\prime}\right)} \cos \left[E_{\vec{p}}\left(t-t_{i}\right)\right] \\
& a_{\pi}\left(\vec{x}-\vec{x}^{\prime}, t\right)=\int d^{3} \vec{x} e^{-i \vec{p} \cdot\left(\vec{x}-\vec{x}^{\prime}\right)} \frac{\sin \left[E_{\vec{p}}\left(t-t_{i}\right)\right]}{E_{\vec{p}}} .
\end{aligned}
$$


Next, let us extract from (4.7) the part which depends only on the initial field configurations which is:

$$
\tilde{\mathcal{Z}}_{\beta}\left[J_{\phi}\right] \equiv \frac{\int \mathscr{D} \phi_{i}(\vec{x}) \mathscr{D} \pi_{i}(\vec{x}) e^{-\beta H_{0}\left(\phi_{i}, \pi_{i}\right)+\int d^{4} x J_{\phi}(x) \phi_{0}(x)}}{\int \mathscr{D} \phi_{i}(\vec{x}) \mathscr{D} \pi_{i}(\vec{x}) e^{-\beta H_{0}\left(\phi_{i}, \pi_{i}\right)}}
$$

where we have switched off [10] the interaction in $H$ so that $H_{0}$ is just:

$$
H_{0}\left(\phi_{i}, \pi_{i}\right)=\int d^{4} x\left[\frac{\pi_{i}^{2}}{2}+\frac{\left(\nabla \phi_{i}\right)^{2}}{2}+\frac{m^{2} \phi_{i}^{2}}{2}\right] .
$$

Inserting (4.10)-(4.12) in (4.13) it is easy to perform the integration over the initial condition (see Ref. [10] for details or Appendix A of our paper) and the result is:

$$
\tilde{z}_{\beta}\left[J_{\phi}\right]=\exp \left[-\frac{1}{2} \int d^{4} x d^{4} x^{\prime} J_{\phi}(x) \Delta_{\beta}\left(x-x^{\prime}\right) J_{\phi}\left(x^{\prime}\right)\right]
$$

where:

$$
\Delta_{\beta}\left(x-x^{\prime}\right)=\int \frac{d^{4} p}{(2 \pi)^{4}} \frac{2 \pi}{\beta\left|p^{0}\right|} \delta\left(p^{2}-m^{2}\right) e^{-i p \cdot\left(x-x^{\prime}\right)} .
$$

It is easy to prove that

$$
\Delta_{\beta}\left(x-x^{\prime}\right)=\Delta_{\beta}\left(x^{\prime}-x\right)
$$

Now, going back to the full expression (4.7) of the generating functional, the full Bosonic part is:

$$
\begin{aligned}
\mathcal{Z}_{(B) \beta}\left[J_{\phi}\right]= & {\left[-\frac{1}{2} \int d^{4} x d^{4} x^{\prime} J_{\phi}(x) \Delta_{\beta}\left(x-x^{\prime}\right) J_{\phi}\left(x^{\prime}\right)\right.} \\
& \left.+i \int d^{4} x d^{4} x^{\prime} J_{\phi}(x) G_{R}^{(B)}\left(x-x^{\prime}\right) J_{\lambda_{\pi}}\left(x^{\prime}\right)\right] .
\end{aligned}
$$

Let us calculate the thermal two-point function

$$
\left\langle\phi\left(x_{2}\right) \phi\left(x_{1}\right)\right\rangle_{\beta}=\left.\left(\frac{1}{i}\right)^{2} \frac{\delta}{\delta J_{\phi}\left(x_{2}\right)} \frac{\delta}{\delta J_{\phi}\left(x_{1}\right)} Z_{\beta}\left[J_{\phi}\right]\right|_{J_{\phi}=0}=\Delta\left(x_{2}-x_{1}\right) .
$$

We can see that this correlation is not anymore the product of the two fields at $x_{2}$ and $x_{1}$ like in (3.19), but it is a function $\Delta_{\beta}\left(x_{2}-x_{1}\right)$ which links $x_{2}$ with $x_{1}$. We will use the full line to indicate the Feynman diagram associated to $\Delta_{\beta}\left(x_{2}-x_{1}\right)$

$$
\Delta_{\beta}\left(x_{2}-x_{1}\right)=\underset{x_{1}}{\bullet} x_{2}
$$

Let us now see how to get the first order correction in $\mathrm{g}$ to the two-point function. Let us, for example, look at the first diagram in (3.35), and let us take its thermal average indicated by \langle\rangle$_{\beta}$ :

$$
\begin{aligned}
& \frac{1}{3 !}\left\langle\underset{x_{1}}{\bullet}\right\rangle_{\beta}=\frac{g}{3 !} \int d y G_{R}\left(x_{1}-y\right)\left\langle\phi_{0}(y)^{3} \phi_{0}\left(x_{2}\right)\right\rangle_{\beta} \\
& =\left.\frac{g}{3 !} \int d y G_{R}\left(x_{1}-y\right) \frac{1}{i^{4}} \frac{\delta^{3}}{\delta J_{\phi}(y)^{3}} \frac{\delta}{\delta J_{\phi}\left(x_{2}\right)} Z_{\beta}\left[J_{\phi}\right]\right|_{J_{\phi}=0} \\
& =\frac{g}{3 !} \int d y G_{R}\left(x_{1}-y\right)\left[3 \Delta_{\beta}(y, y) \Delta_{\beta}\left(y-x_{2}\right)\right] \\
& =\frac{1}{2 !} \stackrel{\longrightarrow}{x_{1}}-\underset{y}{\longrightarrow} x_{2} \text {. }
\end{aligned}
$$


Let us notice that we do not have anymore disconnected diagrams. The three legs in $y$ going nowhere and the one in $x_{2}$ get soldered to each other in all possible combinations producing the loops in (4.21) in $y$ and the propagator between $y$ and $x_{2}$.

The same can be done for the second diagram in (3.35)

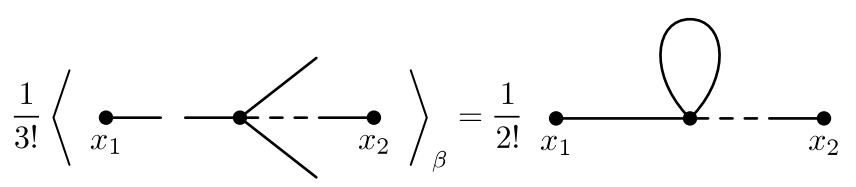

The same for the second order contribution in (3.37). After some long, but straightforward calculations, we get for the first diagram in (3.37):

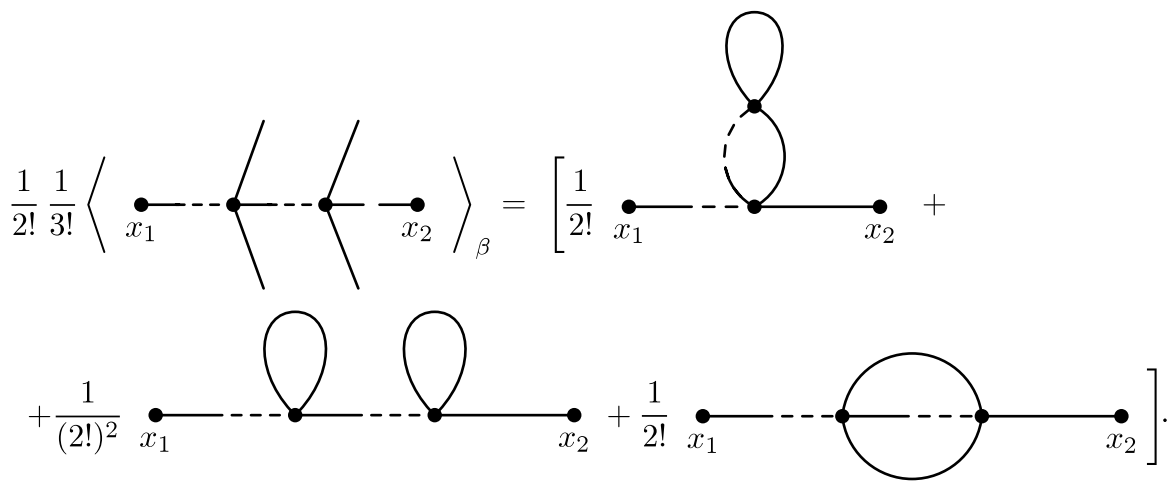

For the second diagram in (3.37) we obtain:

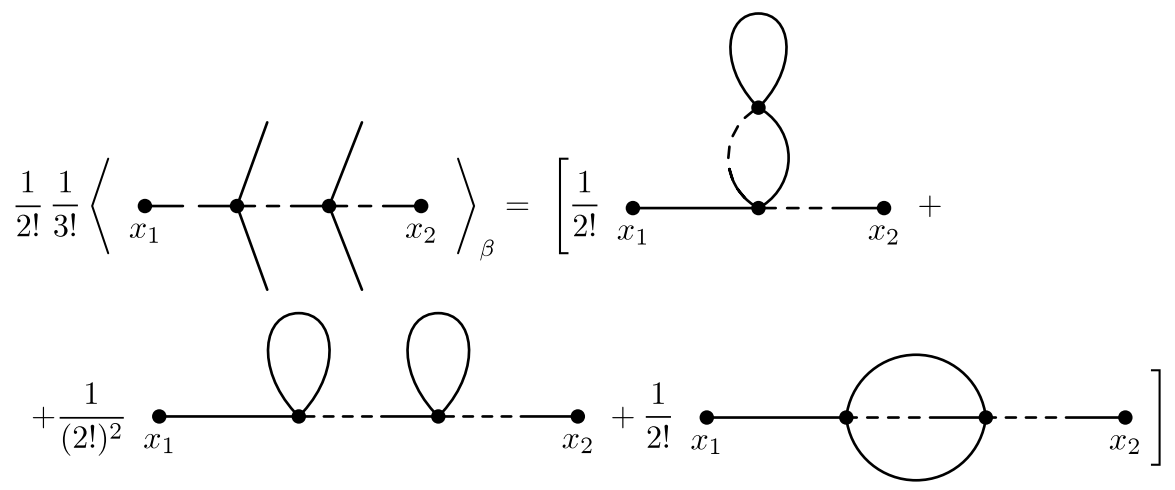

and the third term in (3.37) gives:

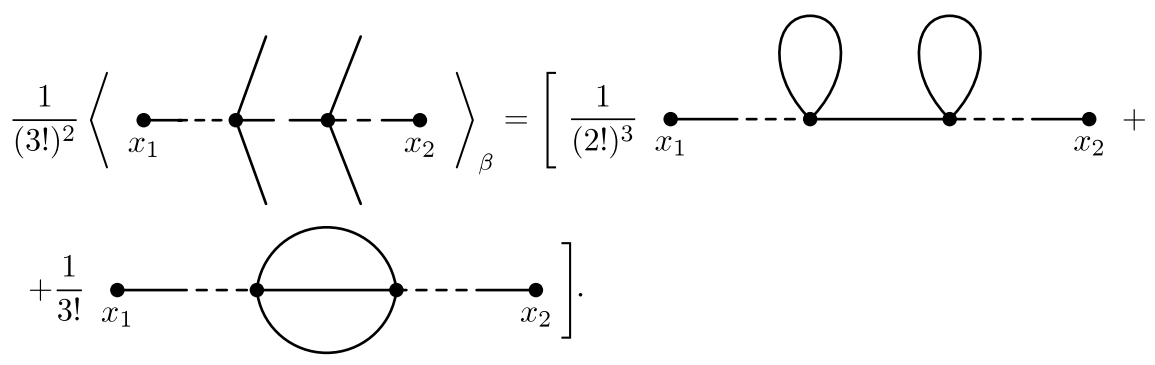


Summing up all the terms up to second order we get:

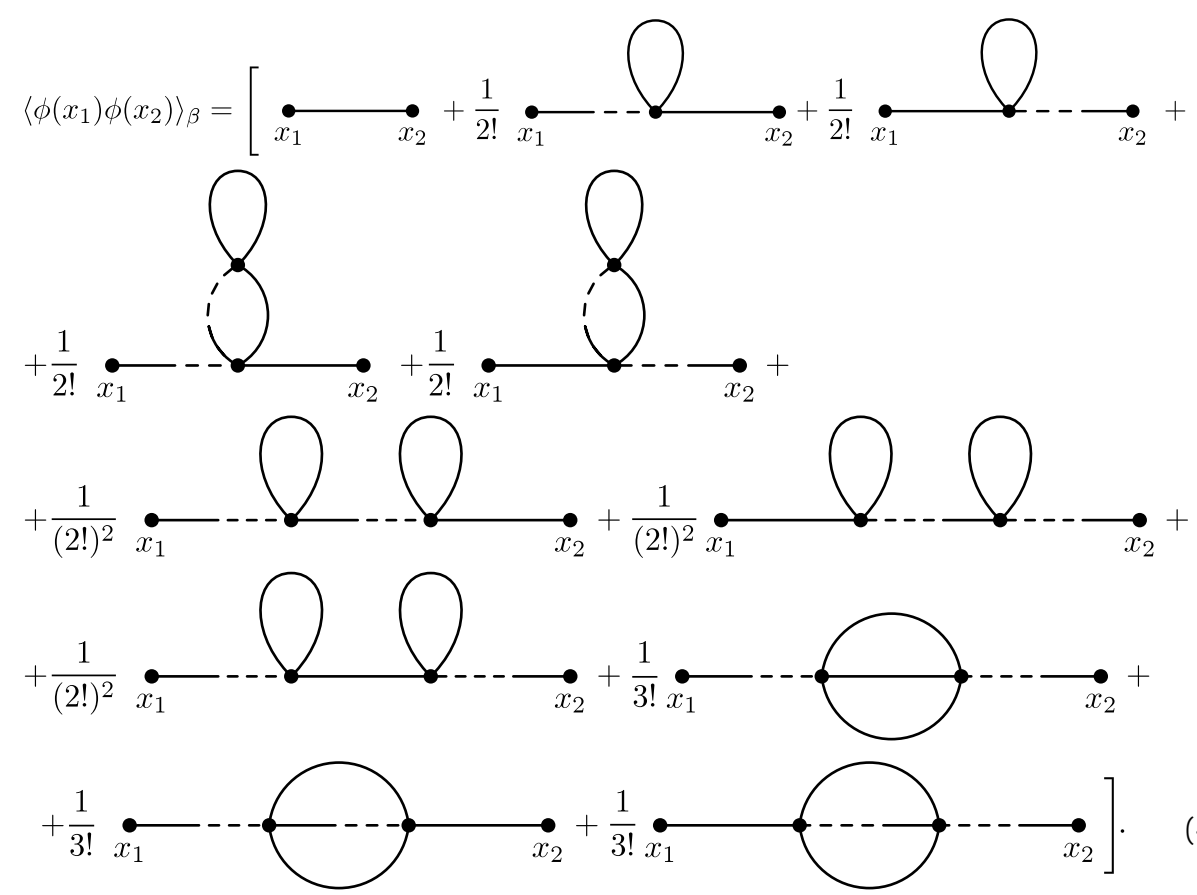

The reader may wonder that there could be some extra diagram coming from the Fermionic part of the generating functional that we have not considered. This is not so because in this calculation we started from the result we had got for the analog diagrams without thermal averages (3.38) and for those diagrams we had already taken account of the Fermionic diagrams cancelation. For example, just using topological considerations, we could have for the two-point function also the following diagrams besides the (4.26)

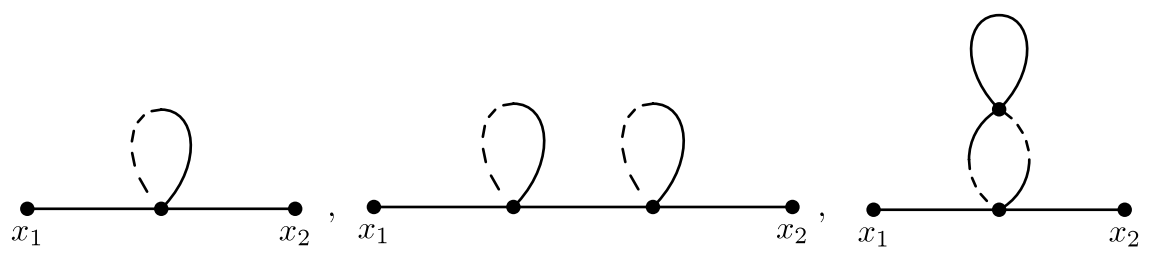

but all these would be canceled by the diagrams with Fermionic loops like:

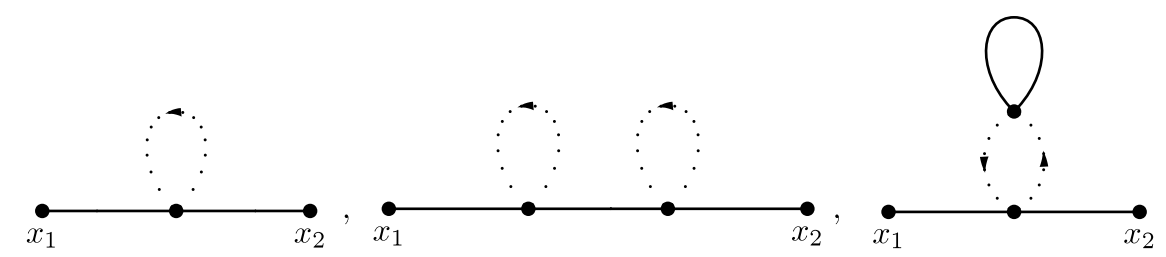

while no diagram in (4.26) could be canceled by diagrams with Fermionic loops.

The diagrams we get by averaging over the initial conditions with the Boltzmann weight are basically based on the following propagators and vertices

$$
\Delta_{\beta}(p)=\frac{2 \pi}{\beta\left|p^{0}\right|} \delta\left(p^{2}-m^{2}\right)=\stackrel{\bullet}{\mathfrak{x}_{1}} \quad \dot{x}_{2}
$$




$$
\begin{aligned}
& -i G_{R}^{(B)}(p)=-\frac{i}{p^{2}-m^{2}+i \epsilon p^{0}}=\stackrel{\bullet}{x_{1}}--\bullet x_{2} \\
& -i G_{A}^{(F)}(p)=-i G_{R}^{(B)}(-p)=\bullet x_{1}--\longrightarrow x_{2}
\end{aligned}
$$

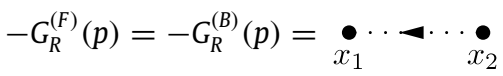

$$
\begin{aligned}
& -G_{A}^{(F)}(p)=-G_{R}^{(B)}(-p)=\bullet_{x_{1}} \cdots \cdot \dot{x}_{2} \\
& i g= \\
& -g=
\end{aligned}
$$

\section{Perturbation theory with super-fields}

If we add to the $\mathcal{Z}_{\beta}^{(B)}$ of (4.18) the Grassmannian piece (3.12) (which is not affected by the thermal average) we get the full generating functional which, for the free theory, is:

$$
\begin{aligned}
\mathcal{Z}_{\beta}^{0}[J]= & {\left[-\frac{1}{2} \int d^{4} x d^{4} x^{\prime} J_{\phi}(x) \Delta_{\beta}\left(x-x^{\prime}\right) J_{\phi}\left(x^{\prime}\right)+i \int d^{4} x d^{4} x^{\prime} J_{\phi}(x) G_{R}^{(B)}\left(x-x^{\prime}\right) J_{\lambda_{\pi}}\left(x^{\prime}\right)\right.} \\
& \left.-\int d^{4} x d^{4} x^{\prime} \bar{J}_{c^{\phi}}(x) G_{R}^{(F)}\left(x-x^{\prime}\right) J_{\bar{c}_{\pi}}\left(x^{\prime}\right)\right] .
\end{aligned}
$$

Let us now move quickly to the super-field formalism. The super-field analog of (2.25) is the following one:

$$
\Phi=\phi+\theta c^{\phi}+\bar{\theta} \bar{c}_{\pi}+i \bar{\theta} \theta \lambda_{\pi}
$$

and in order to have the current-field coupling that we saw in (3.11) and (3.12), we need to introduce a super-current of the form:

$$
\mathbb{J}_{\phi}=-J_{\lambda_{\pi}}+\theta J_{\bar{c}_{\pi}}+\bar{\theta} \bar{J}_{c^{\phi}}-i \bar{\theta} \theta J_{\phi} .
$$

The term:

$$
\exp \left[i \int d^{4} x d \theta d \bar{\theta} \mathbb{J}_{\phi}(x, \theta, \bar{\theta}) \Phi(x, \theta, \bar{\theta})\right]
$$

will then give the four couplings of currents and fields present in (3.11) and (3.12). If we want to get not the free generating functional but the full one, its formal expression will be

$$
\mathcal{Z}\left[\mathbb{J}_{\phi}\right]=\exp \left[\int d z V\left(-\frac{\delta}{\delta \mathbb{J}_{\phi}(z)}\right)\right] Z_{\beta}^{0}\left[\mathbb{J}_{\phi}\right]
$$

where $V$ is the potential of the $g \phi^{4} / 4$ ! theory, so

$$
V\left(-\frac{\delta}{\delta \mathbb{J}_{\phi}(z)}\right)=\frac{g}{4 !} \frac{\delta^{4}}{\delta \mathbb{J}_{\phi}^{4}(z)} \Longrightarrow
$$


where the collective variable $z=(x, \theta, \bar{\theta})$ has been introduced. The reason we can use this potential and not the $\tilde{\delta}_{V}$ of Eq. (3.10) is because, as we proved in Ref. [18], the $\tilde{\vartheta}_{V}$ or any $\tilde{s}$ can be obtained via the usual $\&$ with $\phi$ replaced by $\Phi$ as in formulas (2.18) and (2.19). A super-field formalism and the associated Feynman diagrams had been developed for the Langevin stochastic equation in Ref. [34]. This has inspired us to do an analogous formalism for deterministic systems which evolve via Hamilton's equation.

Going now back to the coupling between super-currents and super-fields in (5.4), it is straightforward to prove that:

$$
\begin{aligned}
J_{\phi} & =i \int d \theta d \bar{\theta} \mathbb{J}_{\phi}(x, \theta, \bar{\theta}) \\
J_{\lambda_{\pi}} & =-\int d \theta d \bar{\theta} \bar{\theta} \theta \mathbb{J}_{\phi}(x, \theta, \bar{\theta}) \\
\bar{J}_{c} & =-\int d \theta d \bar{\theta} \mathbb{J}_{\phi}(x, \theta, \bar{\theta}) \theta \\
J_{\bar{c}_{\pi}} & =\int d \theta d \bar{\theta} \bar{\theta} \mathbb{J}_{\phi}(x, \theta, \bar{\theta}) .
\end{aligned}
$$

Using the rules (5.7)-(5.10) and the super-field we can write the free generating functional (5.1) as

$$
Z_{\beta}^{0}\left[\mathbb{J}_{\phi}\right]=\exp \left[\frac{1}{2} \int d z d z^{\prime} \mathbb{J}_{\phi}(z) \mathbb{G}\left(z, z^{\prime}\right) \mathbb{J}_{\phi}\left(z^{\prime}\right)\right]
$$

where $z=(x, \theta, \bar{\theta})$ and $\mathbb{G}\left(z, z^{\prime}\right)$ is defined as:

$$
\mathbb{G}\left(z, z^{\prime}\right)=\Delta_{\beta}\left(x-x^{\prime}\right)+g\left(z, z^{\prime}\right)=\underset{z}{\stackrel{\bullet}{\bullet}}
$$

with

$$
g\left(z, z^{\prime}\right) \equiv G_{R}^{(B)}\left(x-x^{\prime}\right) \bar{\theta}^{\prime} \theta^{\prime}+G_{R}^{(B)}\left(x^{\prime}-x\right) \bar{\theta} \theta+G_{R}^{(F)}\left(x-x^{\prime}\right) \theta \bar{\theta}^{\prime}+G_{R}^{(F)}\left(x^{\prime}-x\right) \theta^{\prime} \bar{\theta} .
$$

The $\mathbb{G}\left(z, z^{\prime}\right)$ and $g\left(z, z^{\prime}\right)$ have the following nice properties (with $m \geq 1$ ):

1.

$$
\mathbb{G}\left(z, z^{\prime}\right)=\mathbb{G}\left(z^{\prime}, z\right)
$$

2.

$$
\mathbb{G}(z, z)=\Delta_{\beta}(x-x) .
$$

3.

$$
g^{n}\left(z, z^{\prime}\right)=\delta_{n 1} g^{n}\left(z, z^{\prime}\right)\left[1-\delta\left(z-z^{\prime}\right)\right], \quad n \geq 1
$$

4.

$$
\mathbb{G}^{m}\left(z, z^{\prime}\right)=\Delta_{\beta}^{m}\left(x-x^{\prime}\right)+m \Delta_{\beta}^{m-1}\left(x-x^{\prime}\right) \mathcal{g}\left(z, z^{\prime}\right) .
$$

While (1) and (2) are trivial to prove from the symmetry properties of $\Delta_{\beta}$ and $g_{\text {, }}$ (4) can be proved as follows:

$$
\begin{aligned}
\mathbb{G}^{m}\left(z, z^{\prime}\right) & =\sum_{0 \leq n \leq m}\left(\begin{array}{c}
m \\
n
\end{array}\right) \Delta_{\beta}^{m-n}\left(x-x^{\prime}\right) g^{n}\left(z, z^{\prime}\right) \\
& =\left(\begin{array}{c}
m \\
0
\end{array}\right) \Delta_{\beta}^{m}\left(x-x^{\prime}\right)+\left(\begin{array}{c}
m \\
1
\end{array}\right) \Delta_{\beta}^{m-1}\left(x-x^{\prime}\right) g\left(z, z^{\prime}\right) \\
& =\Delta_{\beta}^{m}\left(x-x^{\prime}\right)+m \Delta_{\beta}^{m-1}\left(x-x^{\prime}\right) g\left(z, z^{\prime}\right) .
\end{aligned}
$$


In the last step we have used $g^{n}\left(z, z^{\prime}\right)=0(n \geq 2)$ which is a consequence of the property number (3). Let us now prove property number (3). We have, for $n \geq 1$ :

$$
\begin{aligned}
g^{n}\left(z, z^{\prime}\right) & =g^{n}\left(z, z^{\prime}\right)\left\{\delta\left(z-z^{\prime}\right)+\left[1-\delta\left(z-z^{\prime}\right)\right]\right\} \\
& =g^{n}(z, z) \delta\left(z-z^{\prime}\right)+g^{n}\left(z, z^{\prime}\right)\left[1-\delta\left(z-z^{\prime}\right)\right] \\
& =\left[\delta_{n 1}+\left(1-\delta_{n 1}\right)\right] g^{n}\left(z, z^{\prime}\right)\left[1-\delta\left(z-z^{\prime}\right)\right] \\
& =\delta_{n 1} g^{n}\left(z, z^{\prime}\right)\left[1-\delta\left(z-z^{\prime}\right)\right]+\left(1-\delta_{n 1}\right) g^{n}\left(z, z^{\prime}\right)\left[1-\delta\left(z-z^{\prime}\right)\right] \\
& =\delta_{n 1} g^{n}\left(z, z^{\prime}\right)\left[1-\delta\left(z-z^{\prime}\right)\right] .
\end{aligned}
$$

The property no. (3) is at the root of the fact that loops made of dash-full line cancel against Grassmannian loops. So it is at the root of "classicality", i.e. that without temperature we would not have any loops. For clarity, we remark that to prove property (3) above we made use of $g^{m}\left(z, z^{\prime}\right)=$ $0=g(z, z)(m \geq 2)$.

Going back to the expressions (5.5) and (5.11) we have seen that the Feynman diagrams are the same as those of a $g \phi^{4} / 4$ ! theory, but with the field replaced by super-field, and the propagator replaced by the super $\mathbb{G}\left(z, z^{\prime}\right)$. We call the reader's attention that, in contrast to what happens in standard quantum field theory, where, due to translational invariance, we have $G_{\tilde{F}}\left(z, z^{\prime}\right) \equiv G_{\tilde{F}}\left(z-z^{\prime}\right)$, with $G_{\tilde{F}}$ denoting the Feynman propagator, here we have $\mathbb{G}\left(z, z^{\prime}\right) \neq \mathbb{G}\left(z-z^{\prime}\right)$. That is why we use the notation $\mathbb{G}\left(z, z^{\prime}\right)$ in the perturbative analytic expressions.

Let us start deriving the two-point function:

$$
\mathbb{G}_{\beta}\left(z_{1}, z_{2}\right)=\left\langle\Phi\left(z_{1}\right) \Phi\left(z_{2}\right)\right\rangle_{\beta}=\left.(-i)^{2} \frac{\delta_{\mathbb{J}_{\phi\left(z_{1}\right)}} \delta_{\mathbb{J}_{\phi\left(z_{2}\right)}} \mathcal{Z}\left[\mathbb{J}_{\phi}\right]}{\mathcal{Z}\left[\mathbb{J}_{\phi}\right]}\right|_{\mathbb{J}_{\phi}=0} .
$$

Doing the analog of standard $g \phi^{4} / 4$ ! QFT perturbation theory, we have that the first order correction to the two-point function is

$$
\frac{1}{2} \int d z \mathbb{G}\left(z_{1}, z\right) \mathbb{G}(z, z) \mathbb{G}\left(z, z_{2}\right)=\frac{1}{2} \underset{z_{1}}{\stackrel{z_{2}}{(}}
$$

which is the super-analog of the QFT diagram with the same symmetry factor. The second order correction, following the analogy with standard QFT, which has the three diagrams:

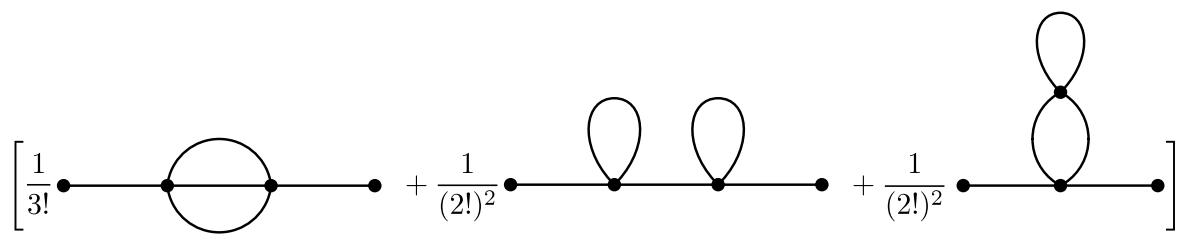

is made of the following three super-diagrams:

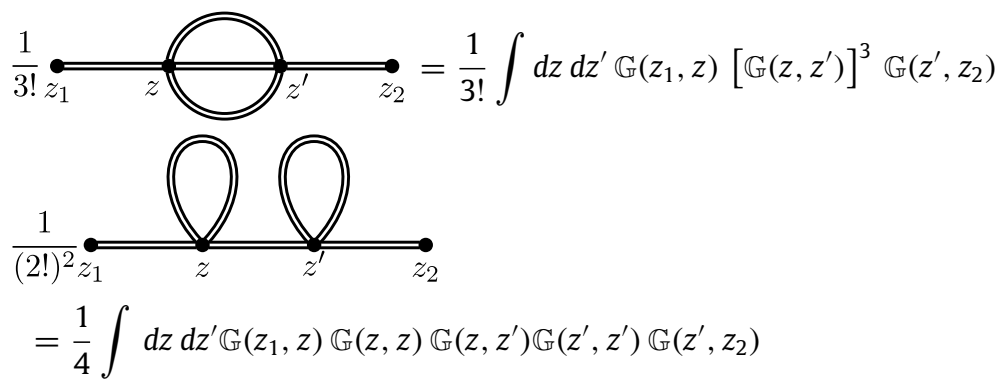




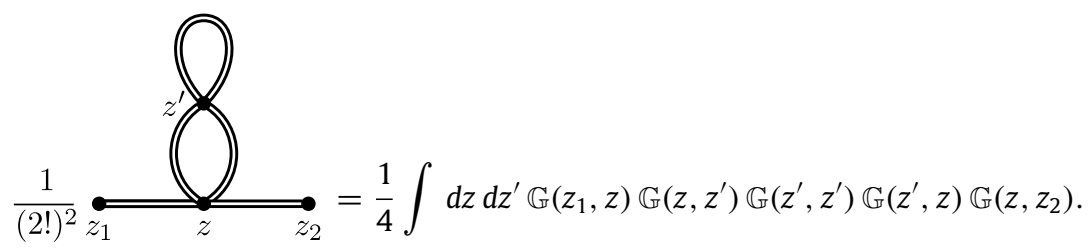

Let us now check if from the super-diagram (5.21) we can derive, for example, the $\langle\phi \phi\rangle$ correlation at the first order which was the sum of (4.21) and (4.22). The manner to do that is to extract from the external super-fields of Eq. (5.21) the components $\varphi^{a}$. Remembering the formula of the super-field (5.2), this can be done via the following integration

$$
\int d \theta_{1} d \bar{\theta}_{1}\left(\bar{\theta}_{1} \theta_{1}\right) d \theta_{2} d \bar{\theta}_{2}\left(\bar{\theta}_{2} \theta_{2}\right)\left[\frac{1}{2 !} \underset{z_{1}}{\longrightarrow}\right.
$$

The variables inside the brackets $\left(\bar{\theta}_{1} \theta_{1}\right)$ and $\left(\bar{\theta}_{2} \theta_{2}\right)$ basically act as projectors from the super-field $\Phi$ to the field $\phi$.

Let us now work out (5.25) in details using (5.21) and the analytic expression of the superpropagators presented on the l.h.s. of (5.13). Below, we will go pedantically through the details of the calculations. We do that in order to allow the reader to become more familiar with the formalism.

The explicit expression of (5.25) turns out to be

$$
\begin{aligned}
& \int d \theta_{1} d \bar{\theta}_{1}\left(\bar{\theta}_{1} \theta_{1}\right) d \theta_{2} d \bar{\theta}_{2}\left(\bar{\theta}_{2} \theta_{2}\right)\left[\frac{1}{2 !} \underset{z_{1}}{\rightleftharpoons} z_{2}\right] \\
& =\frac{1}{2} \int d \theta_{1} d \bar{\theta}_{1}\left(\bar{\theta}_{1} \theta_{1}\right) d \theta_{2} d \bar{\theta}_{2}\left(\bar{\theta}_{2} \theta_{2}\right) \int d^{4} x d \theta d \bar{\theta} \\
& \times\left[\Delta_{\beta}\left(x_{1}-x\right)+G_{R}^{(B)}\left(x-x_{1}\right) \bar{\theta}_{1} \theta_{1}+G_{R}^{(B)}\left(x_{1}-x\right) \bar{\theta} \theta\right. \\
& \left.+G_{R}^{(F)}\left(x-x_{1}\right) \theta \bar{\theta}_{1}+G_{R}^{(F)}\left(x_{1}-x\right) \theta_{1} \bar{\theta}\right] \\
& \times\left[\Delta_{\beta}(x-x)+g(z, z)\right]\left[\Delta_{\beta}\left(x-x_{2}\right)+G_{R}^{(B)}\left(x-x_{2}\right) \bar{\theta}_{2} \theta_{2}\right. \\
& \left.+G_{R}^{(B)}\left(x_{2}-x\right) \bar{\theta} \theta+G_{R}^{(F)}\left(x-x_{2}\right) \theta \bar{\theta}_{2}+G_{R}^{(F)}\left(x_{2}-x\right) \theta_{2} \bar{\theta}\right] \\
& =\frac{1}{2} \int d^{4} x d \theta d \bar{\theta}\left[\Delta_{\beta}\left(x_{1}-x\right)+G_{R}^{(B)}\left(x_{1}-x\right) \bar{\theta} \theta\right] \\
& \times \Delta_{\beta}\left(x-x^{\prime}\right)\left[\Delta_{\beta}\left(x-x_{2}\right)+G_{R}^{(B)}\left(x_{2}-x\right) \bar{\theta} \theta\right] \\
& =\left[\frac{1}{2 !} \stackrel{\bullet}{x_{1}}-\stackrel{x}{2}_{2 !} \stackrel{1}{x_{1}}\right.
\end{aligned}
$$

and these last diagrams are exactly the sum of (4.21) and (4.22) which were the first order correction to the $\langle\phi \phi\rangle$ propagator. So the lesson we learn from here is the following: it is enough to project the external super-legs of the super-diagrams on the fields we want, in order to get also the correct internal part of the super-diagrams.

Let us now check the second order. That means let us check if the three super-diagrams in Eqs. (5.22)-(5.24), once the external legs are projected on $\phi$, reproduce the eight second order diagrams contained in (4.26). 
Again, in order to get the reader familiar with this formalism, let us calculate explicitly here the first of the three super-diagrams in (5.22), while the calculation of the other two will be confined in Appendix B

$$
\begin{aligned}
& \int d \theta_{1} d \bar{\theta}_{1} d \theta_{2} d \bar{\theta}_{2}\left(\bar{\theta}_{1} \theta_{1}\right)\left(\bar{\theta}_{2} \theta_{2}\right)\left[\frac{1}{3 !} \underset{z_{1}}{\rightleftharpoons}\right. \\
& =\int d \theta_{1} d \bar{\theta}_{1} d \theta_{2} d \bar{\theta}_{2}\left(\bar{\theta}_{1} \theta_{1}\right)\left(\bar{\theta}_{2} \theta_{2}\right) \int d^{4} x d \theta d \bar{\theta} \int d^{4} x^{\prime} d \theta^{\prime} d \bar{\theta}^{\prime}\left[\Delta_{\beta}\left(x_{1}-x\right)\right. \\
& \left.+G_{R}^{(B)}\left(x_{1}-x\right) \bar{\theta} \theta+G_{R}^{(B)}\left(x-x_{1}\right) \bar{\theta}_{1} \theta_{1}+G_{R}^{(F)}\left(x_{1}-x\right) \theta_{1} \bar{\theta}+G_{R}^{(F)}\left(x-x_{1}\right) \theta \bar{\theta}_{1}\right] \\
& \times\left[\Delta_{\beta}\left(x-x^{\prime}\right)+g\left(z, z^{\prime}\right)\right]^{3}\left[\Delta_{\beta}\left(x^{\prime}-x_{2}\right)+G_{R}^{(B)}\left(x^{\prime}-x_{2}\right) \bar{\theta}_{2} \theta_{2}\right. \\
& \left.+G_{R}^{(B)}\left(x_{2}-x^{\prime}\right) \bar{\theta}^{\prime} \theta^{\prime}+G_{R}^{(F)}\left(x^{\prime}-x_{2}\right) \theta^{\prime} \bar{\theta}_{2}+G_{R}^{(F)}\left(x_{2}-x^{\prime}\right) \theta_{2} \bar{\theta}^{\prime}\right] \\
& =\int d \theta_{1} d \bar{\theta}_{1} d \theta_{2} d \bar{\theta}_{2}\left(\bar{\theta}_{1} \theta_{1}\right)\left(\bar{\theta}_{2} \theta_{2}\right) \int d^{4} x d \theta d \bar{\theta} \int d^{4} x^{\prime} d \theta^{\prime} d \bar{\theta}^{\prime} \\
& \times\left[\Delta_{\beta}\left(x_{1}-x\right)+G_{R}^{(B)}\left(x_{1}-x\right) \bar{\theta} \theta+G_{R}^{(B)}\left(x-x_{1}\right) \bar{\theta}_{1} \theta_{1}+G_{R}^{(F)}\left(x_{1}-x\right) \theta_{1} \bar{\theta}\right. \\
& \left.+G_{R}^{(F)}\left(x-x_{1}\right) \theta \bar{\theta}_{1}\right]\left[\Delta_{\beta}^{3}\left(x-x^{\prime}\right)+3 \Delta_{\beta}^{2}\left(x-x^{\prime}\right) g\left(z, z^{\prime}\right)\right] \\
& \times\left[\Delta_{\beta}\left(x^{\prime}-x_{2}\right)+G_{R}^{(B)}\left(x^{\prime}-x_{2}\right) \bar{\theta}_{2} \theta_{2}+G_{R}^{(B)}\left(x_{2}-x^{\prime}\right) \bar{\theta}^{\prime} \theta^{\prime}\right. \\
& \left.+G_{R}^{(F)}\left(x^{\prime}-x_{2}\right) \theta^{\prime} \bar{\theta}_{2}+G_{R}^{(F)}\left(x_{2}-x^{\prime}\right) \theta_{2} \bar{\theta}^{\prime}\right] \\
& =\frac{1}{2} \int d^{4} x d^{4} x^{\prime} \Delta_{\beta}\left(x_{1}-x\right) \Delta_{\beta}^{2}\left(x-x^{\prime}\right) G_{R}^{(B)}\left(x^{\prime}-x\right) G_{R}^{(B)}\left(x_{2}-x^{\prime}\right) \\
& +\frac{1}{2} \int d^{4} x d^{4} x^{\prime} G_{R}^{(B)}\left(x_{1}-x\right) \Delta_{\beta}^{2}\left(x-x^{\prime}\right) G_{R}^{(B)}\left(x-x^{\prime}\right) \Delta_{\beta}\left(x^{\prime}-x_{2}\right) \\
& +\frac{1}{6} \int d^{4} x d^{4} x^{\prime} G_{R}^{(B)}\left(x_{1}-x\right) \Delta_{\beta}^{3}\left(x-x^{\prime}\right) G_{R}^{(B)}\left(x_{2}-x^{\prime}\right)
\end{aligned}
$$

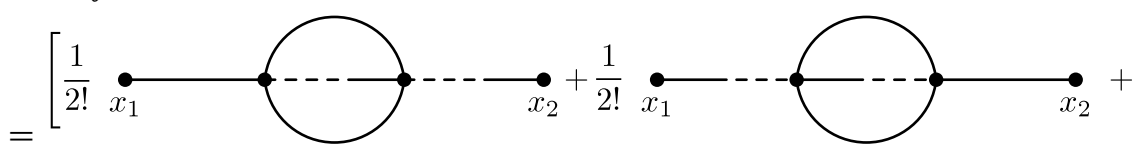

$$
\begin{aligned}
& \left.+\frac{1}{3 !} \stackrel{\bullet}{x_{1}}--\longrightarrow \longrightarrow x_{2}\right] \text {. }
\end{aligned}
$$

These are exactly the last three diagrams of (4.26) and exactly with the same coefficients.

Analogously we can prove (see Appendix B) that

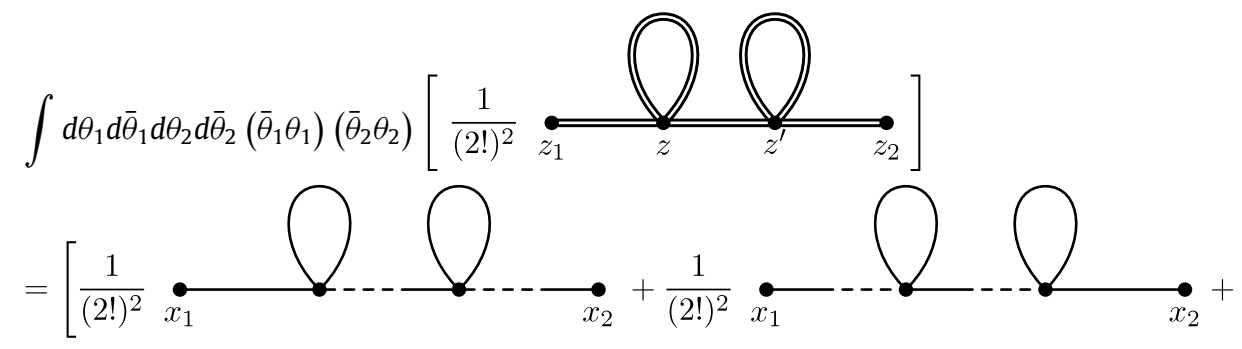




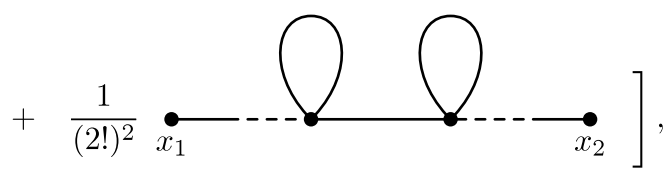

which are the 6th, 7th, 8th diagrams presented in (4.26).

Along the same lines we can easily get (see Appendix B)

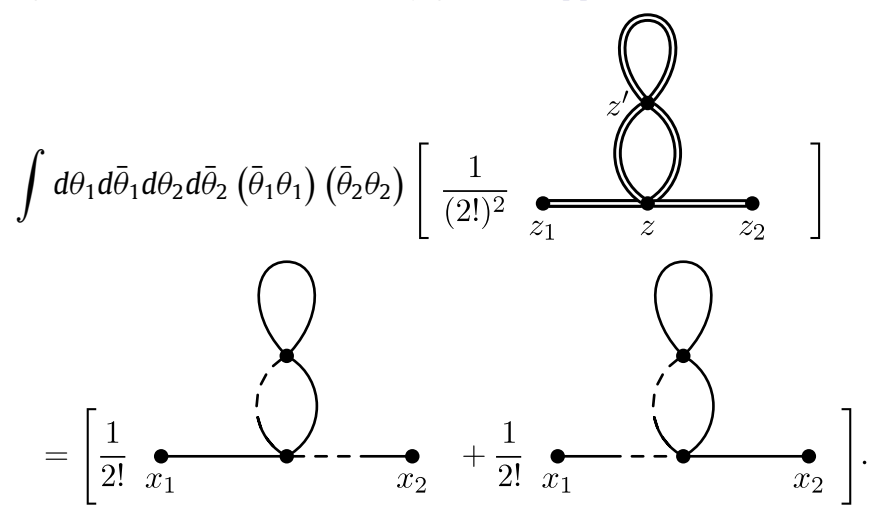

Summing up (5.29), (5.30) and (5.27) we get all the second order diagrams contained in Eq. (4.26). These last ones were eight in number and we got them from just the three super-diagrams in Eqs. (5.22)-(5.24). The decrease in the number of diagrams is one of the virtues of the super-diagram technique. A second virtue is that we only have to perform integrations over the Grassmannian variables (which are easy to do) to get all the old diagrams of $\tilde{\mathscr{L}}$ without bothering with their complicated symmetry factors, without bothering with soldering in the correct manner the legs and vertices of $\tilde{\mathscr{L}}$ and so on. Above all, the main advantage of the super-diagrams is that they can be derived from the analog quantum theory whose Lagrangian $L$ is in general simpler than $\tilde{\mathcal{L}}$. So, once we have the quantum theory and its associated Feynman diagrams and symmetry factors, we are already more than halfway also with the perturbation theory of the associated classical theory. We have just to substitute the fields with the super-fields and the propagators with the super-propagators and perform, as we said above, some very simple Grassmannian integrations. Moreover, the super-diagram automatically perform for us the cancelation between Fermion and dash-full loops.

Last, but not least, by changing the projectors on the external legs, we can get not only $\langle\varphi \varphi\rangle$ correlations, but also $\langle\lambda \varphi\rangle$, the $\langle\bar{c} c\rangle$, the $\langle c c\rangle$, the $\langle\bar{c} \bar{c}\rangle$ and the $\langle\lambda \lambda\rangle$ correlations. So just three second order super-diagrams will produce tens of standard diagrams.

We will start now with the $\left\langle\phi \lambda_{\pi}\right\rangle$ correlations. Looking at the super-field expression in (5.2), we realize immediately that, differently than the $\langle\phi \phi\rangle$ correlation, we have to project out only the $\phi\left(x_{1}\right)$ field because the $\lambda_{\pi}\left(x_{2}\right)$ is already equipped with its own $\bar{\theta}_{2} \theta_{2}$ and so it naturally makes its appearance once we integrate over the final points. From the vertices and propagators that we can build from the formalism in components, it is easy to see that $\left\langle\phi \lambda_{\pi}\right\rangle$ correlation will have the zero order component of the form:

$$
\left\langle\phi\left(x_{1}\right) \lambda_{\pi}\left(x_{2}\right)\right\rangle_{0}=\stackrel{\bullet--\bullet}{x_{1}}
$$

While the first order correction is:

$$
\begin{aligned}
\left\langle\phi\left(x_{1}\right) \lambda_{\pi}\left(x_{2}\right)\right\rangle_{1} & =\int d \theta_{1} d \bar{\theta}_{1} d \theta_{2} d \bar{\theta}_{2}\left(-i \bar{\theta}_{1} \theta_{1}\right)\left[\frac{1}{2 !} \underset{z_{1}}{\bullet}\right. \\
& =\frac{1}{2 !} \bullet_{x_{1}}
\end{aligned}
$$


Here we have used the projector $\left(-i \bar{\theta}_{1} \theta_{1}\right)$ in order to project $\phi\left(x_{1}\right)$ out of the super-field.

The second order corrections have the form:

$$
\left\langle\phi\left(x_{1}\right) \lambda_{\pi}\left(x_{2}\right)\right\rangle_{2}=\int d \theta_{1} d \bar{\theta}_{1} d \theta_{2} d \bar{\theta}_{2}\left(-i \bar{\theta}_{1} \theta_{1}\right)
$$

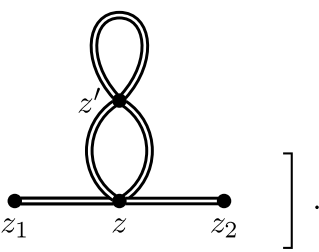

By long but straightforward calculations similar to those performed in Appendix B, the result that we get for the diagrams in (5.33) is the following one:

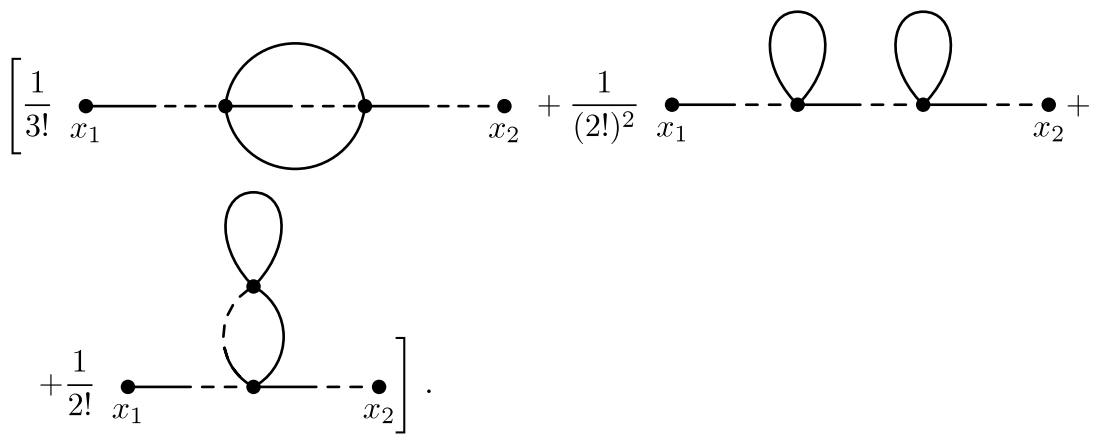

We checked and found out that the diagrams on the r.h.s. of (5.34) are exactly those that we would have obtained using the component formalism with the rules given in Eqs. (4.28)-(4.34) and taking into account the cancellations between dash-full propagators and Fermionic ones.

From now on, we will trust the results given by super-fields once they are properly projected on the component fields we are interested in. We will trust them because they seem to give the same results as if we have done the calculations in components using the rules in Eqs. (4.28)-(4.34).

Let us now calculate the Grassmannian variables correlations. Let us start from the correlation $\left\langle c^{\phi}\left(x_{1}\right) \bar{c}_{\pi}\left(x_{2}\right)\right\rangle$. The zero order is given in Eq. (4.30). The first order, via super-fields, will be obtained from the super-diagram of Eq. (5.21) by projecting out the $c^{\phi}\left(x_{1}\right)$ and $\bar{c}_{\pi}\left(x_{2}\right)$. This is achieved with the projector $\theta_{2} \bar{\theta}_{1}$. The first one extracts from the super-field the field $\bar{c}_{\pi}\left(x_{2}\right)$, while the second one extracts the $c^{\phi}\left(x_{1}\right)$. So, what we have to do is:

$$
\int d \theta_{1} d \bar{\theta}_{1} d \theta_{2} d \bar{\theta}_{2}\left(\theta_{2} \bar{\theta}_{1}\right)\left[\frac{1}{2 !} \underset{z}{z_{1}} \underset{z}{2}\right]
$$

Performing the long but straightforward Grassmannian integration above, we get:

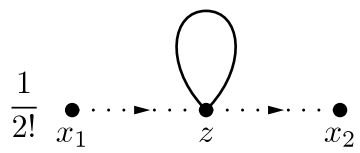

For the second order we just have to perform the following calculations:

$$
\int d \theta_{1} d \bar{\theta}_{1} d \theta_{2} d \bar{\theta}_{2}\left(\theta_{2} \bar{\theta}_{1}\right)\left[\frac{1}{3 !} \underset{z_{1}}{\rightleftharpoons}\right.
$$




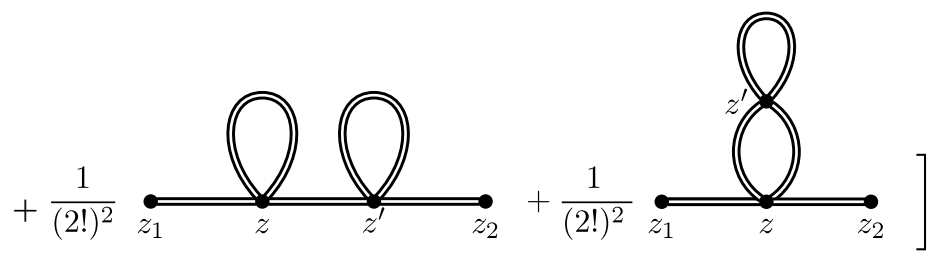

and the result is:

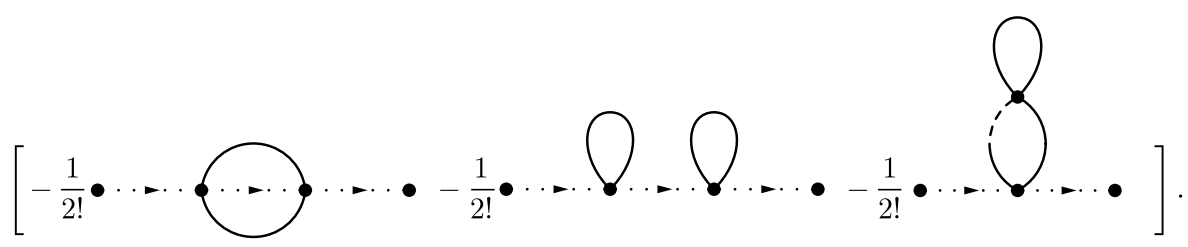

We leave this calculation to the reader and we let him compare it with the calculations in components which follow from the rules stated in Eqs. (4.28)-(4.30), (4.32) and (4.33).

Next, let us calculate

$$
\left\langle c^{\phi}\left(x_{1}\right) c^{\phi}\left(x_{2}\right)\right\rangle_{\beta} \text {. }
$$

The projector in this case is $\left(\bar{\theta}_{1} \bar{\theta}_{2}\right)$ :

$$
\int d \theta_{1} d \bar{\theta}_{1} d \theta_{2} d \bar{\theta}_{2}\left(\bar{\theta}_{1} \bar{\theta}_{2}\right)\left\langle\Phi\left(z_{1}\right) \Phi\left(z_{2}\right)\right\rangle_{\beta}=\left\langle c^{\phi}\left(x_{1}\right) c^{\phi}\left(x_{2}\right)\right\rangle_{\beta} .
$$

By an argument presented in Appendix D we can prove, to all orders in perturbation theory, that:

$$
\left\langle c^{\phi}\left(x_{1}\right) c^{\phi}\left(x_{2}\right)\right\rangle_{\beta}=0 .
$$

With similar arguments we can also prove that

$$
\left\langle\phi\left(x_{1}\right) c^{\phi}\left(x_{2}\right)\right\rangle_{\beta}=\left\langle\phi\left(x_{1}\right) \bar{c}_{\pi}\left(x_{2}\right)\right\rangle_{\beta}=\left\langle\lambda\left(x_{1}\right) c^{\phi}\left(x_{2}\right)\right\rangle_{\beta}=\left\langle\lambda\left(x_{1}\right) \bar{c}_{\pi}\left(x_{2}\right)\right\rangle_{\beta}=0 .
$$

Let us just work out the first one of the relations in (5.40). The projector needed to derive the desired correlation from the super-fields is $\left(\bar{\theta}_{2} \bar{\theta}_{1} \theta_{1}\right)$, so:

$$
\begin{aligned}
\int & d \theta_{1} d \bar{\theta}_{1} d \theta_{2} d \bar{\theta}_{2}\left(\bar{\theta}_{2} \bar{\theta}_{1} \theta_{1}\right)\left\langle\Phi\left(z_{1}\right) \Phi\left(z_{2}\right)\right\rangle_{\beta} \\
= & \int d \theta_{1} d \bar{\theta}_{1} d \theta_{2} d \bar{\theta}_{2}\left(\bar{\theta}_{2} \bar{\theta}_{1} \theta_{1}\right) \int d z d z^{\prime} \mathbb{G}\left(z_{1}, z\right) \mathbb{F}^{(n)}\left(z, z^{\prime}\right) \mathbb{G}\left(z^{\prime}, z_{2}\right) \\
= & \int d \theta_{1} d \bar{\theta}_{1} d \theta_{2} d \bar{\theta}_{2}\left(\bar{\theta}_{2} \bar{\theta}_{1} \theta_{1}\right) \int d^{4} x d \theta d \bar{\theta} \int d^{4} x^{\prime} d \theta^{\prime} d \bar{\theta}^{\prime}\left[\Delta_{\beta}\left(x_{1}-x\right)+G_{R}^{(B)}\left(x_{1}-x\right) \bar{\theta} \theta\right. \\
& \left.+G_{R}^{(B)}\left(x-x_{1}\right) \bar{\theta}_{1} \theta_{1}+G_{R}^{(F)}\left(x_{1}-x\right) \theta_{1} \bar{\theta}+G_{R}^{(F)}\left(x-x_{1}\right) \theta \bar{\theta}_{1}\right] \mathbb{F}^{(n)}\left(z, z^{\prime}\right) \\
& \times\left[\Delta_{\beta}\left(x^{\prime}-x_{2}\right)+G_{R}^{(B)}\left(x^{\prime}-x_{2}\right) \bar{\theta}_{2} \theta_{2}+G_{R}^{(B)}\left(x_{2}-x^{\prime}\right) \bar{\theta}^{\prime} \theta^{\prime}\right. \\
& \left.+G_{R}^{(F)}\left(x^{\prime}-x_{2}\right) \theta^{\prime} \bar{\theta}_{2}+G_{R}^{(F)}\left(x_{2}-x^{\prime}\right) \theta_{2} \bar{\theta}^{\prime}\right] \\
\equiv & \mathbb{A}+\mathbb{B} .
\end{aligned}
$$

where $\mathbb{F}^{(n)}$ is defined in Appendix $C$, while $\mathbb{A}$ and $\mathbb{B}$ are:

$$
\mathbb{A} \equiv \int d z d z^{\prime} \bar{\theta}^{\prime} \Delta_{\beta}\left(x_{1}-x\right) \mathbb{F}^{(n)}\left(z, z^{\prime}\right) G_{R}^{(F)}\left(x_{2}-x^{\prime}\right)
$$


and

$$
\mathbb{B} \equiv \int d z d z^{\prime} \bar{\theta} \theta \bar{\theta}^{\prime} G_{R}^{(F)}\left(x_{1}-x\right) \mathbb{F}^{(n)}\left(z, z^{\prime}\right) G_{R}^{(F)}\left(x_{2}-x^{\prime}\right)
$$

In Appendix $C$ we proved that the Grassmannian coefficients of $\mathbb{F}^{(n)}$ can only have the following six forms: (1) $\theta \bar{\theta} \theta^{\prime} \bar{\theta}^{\prime}$, (2) $\theta \bar{\theta}$, (3) $\theta^{\prime} \bar{\theta}^{\prime}$, (4) $\theta \bar{\theta}^{\prime}$, (5) $\bar{\theta} \theta^{\prime}$ and (6) II. Inserting any of them in $\mathbb{A}$ or $\mathbb{B}$ we get that $\mathbb{A}=0=\mathbb{B}$ in all cases and this proves the first identity in Eq. (5.40). The proof of the other relations in Eq. (5.40) are analogous and we leave them to the reader.

Let us now prove that, to all orders in perturbation theory, we have:

$$
\left\langle\lambda_{\pi}\left(x_{1}\right) \lambda_{\pi}\left(x_{2}\right)\right\rangle_{\beta}=0 .
$$

To simplify the calculations let us use the notation $d \mu \equiv d \theta d \bar{\theta}$. In order to project out the $\lambda$ field from the super-field we need, as projector, the operator $-\mathbb{I}$, so

$$
\int d \theta_{1} d \bar{\theta}_{1} d \theta_{2} d \bar{\theta}_{2}(-\mathbb{I})\left\langle\Phi\left(z_{1}\right) \Phi\left(z_{2}\right)\right\rangle_{\beta} \equiv\left\langle\lambda_{\pi}\left(x_{1}\right) \lambda_{\pi}\left(x_{2}\right)\right\rangle_{\beta}
$$

Let us now consider (5.45) at $n$-th order in perturbation theory

$$
\begin{aligned}
\int & d \theta_{1} d \bar{\theta}_{1} d \theta_{2} d \bar{\theta}_{2}(-\mathbb{I})\left\langle\Phi\left(z_{1}\right) \Phi\left(z_{2}\right)\right\rangle_{\beta}^{(n)} \\
= & -\int d \theta_{1} d \bar{\theta}_{1} d \theta_{2} d \bar{\theta}_{2} \int d z d z^{\prime} \mathbb{G}\left(z_{1}, z\right) \mathbb{F}^{(n)}\left(z, z^{\prime}\right) \mathbb{G}_{\left(z^{\prime}, z_{2}\right)} \\
= & -\int d \theta_{1} d \bar{\theta}_{1} d \theta_{2} d \bar{\theta}_{2} \int d^{4} x d \theta d \bar{\theta} \int d^{4} x^{\prime} d \theta^{\prime} d \bar{\theta}^{\prime}\left[\Delta_{\beta}\left(x_{1}-x\right)+G_{R}^{(B)}\left(x_{1}-x\right) \bar{\theta} \theta\right. \\
& \left.+G_{R}^{(B)}\left(x-x_{1}\right) \bar{\theta}_{1} \theta_{1}+G_{R}^{(F)}\left(x_{1}-x\right) \theta_{1} \bar{\theta}+G_{R}^{(F)}\left(x-x_{1}\right) \theta \bar{\theta}_{1}\right] \mathbb{F}^{(n)}\left(z, z^{\prime}\right) \\
& \times\left[\Delta_{\beta}\left(x^{\prime}-x_{2}\right)+G_{R}^{(B)}\left(x^{\prime}-x_{2}\right) \bar{\theta}_{2} \theta_{2}+G_{R}^{(B)}\left(x_{2}-x^{\prime}\right) \bar{\theta}^{\prime} \theta^{\prime}\right. \\
& \left.+G_{R}^{(F)}\left(x^{\prime}-x_{2}\right) \theta^{\prime} \bar{\theta}_{2}+G_{R}^{(F)}\left(x_{2}-x^{\prime}\right) \theta_{2} \bar{\theta}^{\prime}\right] \\
= & -\int d z d z^{\prime} \int d \theta_{1} d \bar{\theta}_{1} d \theta_{2} d \bar{\theta}_{2}\left[G_{R}^{(B)}\left(x-x_{1}\right) \bar{\theta}_{1} \theta_{1}\right] \mathbb{F}^{(n)}\left(z, z^{\prime}\right)\left[G_{R}^{(B)}\left(x^{\prime}-x_{2}\right) \bar{\theta}_{2} \theta_{2}\right] \\
= & -\int d^{4} x d^{4} x^{\prime} G_{R}^{(B)}\left(x-x_{1}\right) G_{R}^{(B)}\left(x^{\prime}-x_{2}\right)\left[\int d \mu d \mu^{\prime} \mathbb{F}^{(n)}\left(z, z^{\prime}\right)\right] .
\end{aligned}
$$

In Appendix C we will prove several identities which will lead to

$$
\int d \mu d \mu^{\prime} \mathbb{F}^{(n)}\left(z, z^{\prime}\right)=0
$$

Because of this, from (5.46) we obtain, to all orders, that:

$$
\left\langle\lambda_{\pi}\left(x_{1}\right) \lambda_{\pi}\left(x_{2}\right)\right\rangle_{\beta}=0 .
$$

The correlation above is zero not only because of the identities that we proved but also because we somehow are using here the closed-time-path (CTP) formalism for classical thermal field theory [31]. We will not expand on this here but advice the interested reader to read Ref. [31].

For the correlation $\left\langle\phi\left(x_{2}\right) \phi\left(x_{1}\right)\right\rangle_{\beta}$ we have calculated things up to second order which is what has been done in the literature up to now. We will show in Appendix $E$ that with our super-field technique it is not difficult to reach the third order. 


\section{Fluctuation-dissipation theorem}

This is a well-known theorem that holds for many systems like those obeying the Langevin equation and similar equations. It basically relates the two-point function (fluctuation) of the system to the manner the system responds to external perturbation (dissipation). It was proved to hold [15] also for deterministic systems whose initial distribution was the Boltzmann one, so it should hold also for the approach to classical field theory with temperature that we have explored in this paper.

Several years ago it was showed [28] that the fluctuation-dissipation theorem (FDT) for Langevin equation could be proved non-perturbatively as a "Ward identity" of a hidden supersymmetry present in the Langevin equation. As that supersymmetry is very similar to the one we have in the classical formalism presented here, also in the classical case the FDT can be derived as a Ward identity of the supersymmetry. In Appendix $\mathrm{F}$ we will present that non-perturbative derivation generously provided to us by Martin Reuter. Of course, the FDT for deterministic systems can be proved in a much simpler way [15] than going through the Ward identities of Susy, nevertheless, we like to look at it as a Ward identity because it actually relates different correlation functions like Ward identities do.

For the moment, anyhow, let us leave aside its relations to Susy and Ward identities and let us start verifying its validity at the perturbative level, so that we can test the tools developed in this paper. Let us follow the notation of Ref. [15], that from now on we will indicate with the initial letters "DH", while we will indicate ours by the acronym CPI. In the table below we write down the correspondence between the notation of $\mathrm{DH}$ and the one of the CPI. We urge the reader to read Ref. [15] in order to understand the symbols contained in the table below:

\begin{tabular}{|c|c|}
\hline$[\mathrm{DH}]$ & {$[\mathrm{CPI}]$} \\
\hline$D_{i j}$ & $\omega^{a b}$ \\
\hline$R_{i j}\left(t-t^{\prime}\right)$ & $-i \theta\left(t-t^{\prime}\right)\left\langle\varphi^{a}(t) \lambda_{b}\left(t^{\prime}\right)\right\rangle$ \\
\hline$C_{i j}\left(t-t^{\prime}\right)$ & $\left\langle\varphi^{a}(t) \varphi^{b}\left(t^{\prime}\right)\right\rangle$ \\
\hline$\psi_{i}$ & $\varphi^{a}$ \\
\hline$\hat{\psi}_{i} \equiv-\frac{\partial}{\partial \psi_{i}}$ & $-i \lambda_{a}$ \\
\hline
\end{tabular}

The $\varphi^{a}$ indicates all the phase-space coordinates. According to Eq. (2.34) of DH the FDT is

$$
R_{i j}(t)=-\beta \theta(t) \frac{\partial}{\partial t} C_{i \bar{k}} D_{\overline{k j}},
$$

where we follow the convention adopted in DH: the bar over an index, like $\bar{k}$, means they are summed over.

Eq. (6.1) can be written in an alternative form as

$$
\frac{\partial}{\partial t} C_{i j}(t)=\frac{1}{\beta} R_{i \bar{k}}(t) D_{\bar{k} j}-\frac{1}{\beta} D_{\bar{k} i} R_{j \bar{k}}(-t) .
$$

We can prove it in the following way. For $t>0$, Eq. (6.1) becomes

$$
R_{i j}(t)=-\beta \frac{\partial}{\partial t} C_{i \bar{k}} D_{\bar{k} j}
$$

If we multiply the relation above by $D$ we get:

$$
\frac{1}{\beta} R_{i \bar{\ell}}(t) D_{\bar{\ell} j}=-\frac{\partial}{\partial t} C_{i \bar{k}} D_{\bar{k} \bar{\ell}} D_{\bar{\ell} j} \text {. }
$$

Next, using the fact that $D_{k \bar{\ell}} D_{\bar{\ell} j}=-\delta_{k j}$, we obtain:

$$
\frac{\partial}{\partial t} C_{i j}(t)=\frac{1}{\beta} R_{i \bar{k}}(t) D_{\bar{k} j} .
$$


For $t<0$ Eq. (6.1) gives

$$
\begin{aligned}
R_{j i}(-t) & =-\beta \theta(-t) \frac{\partial}{\partial(-t)} C_{j \bar{k}}(-t) D_{\bar{k} i} \\
& =\beta \theta(-t) \frac{\partial}{\partial t} C_{\bar{k} j}(t) D_{\bar{k} i}
\end{aligned}
$$

where we have used the fact that

$$
\frac{\partial}{\partial t} C_{j k}(t)=-\frac{\partial}{\partial t} C_{k j}(-t)
$$

Multiplying (6.7) by $D$ and operating as before, we get for $t<0$

$$
\frac{\partial}{\partial t} C_{i j}(t)=-\frac{1}{\beta} D_{\bar{k} i} R_{j \bar{k}}(-t) .
$$

Collecting (6.5) and (6.8) for $t \gtrless 0$ we get exactly (6.2).

Making use of the table of comparison between DH and CPI, we can turn (6.2) into the following relation

$$
\begin{aligned}
\frac{\partial}{\partial t_{1}}\left\langle\varphi^{a}\left(t_{1}\right) \varphi^{b}\left(t_{2}\right)\right\rangle= & \frac{1}{\beta} \theta\left(t_{1}-t_{2}\right)\left\langle\varphi^{a}\left(t_{1}\right)(-i) \lambda_{d}\left(t_{2}\right)\right\rangle \omega^{d b} \\
& -\frac{1}{\beta} \theta\left(t_{2}-t_{1}\right) \omega^{d a}\left\langle\varphi^{b}\left(t_{2}\right)(-i) \lambda_{d}\left(t_{1}\right)\right\rangle .
\end{aligned}
$$

This is the full fluctuation-dissipation theorem.

Let us choose $a=b=1$ in Eq. (6.9). We will then obtain for a field theory, where $\varphi^{1}=\phi$ and $\varphi^{2}=\pi$, the relation:

$$
\frac{\partial}{\partial t_{1}}\left\langle\varphi^{a}\left(t_{1}\right) \varphi^{b}\left(t_{2}\right)\right\rangle=i \frac{1}{\beta}\left[\theta\left(t_{1}-t_{2}\right)\left\langle\phi\left(t_{1}\right) \lambda_{\pi}\left(t_{2}\right)\right\rangle-\theta\left(t_{2}-t_{1}\right)\left\langle\phi\left(t_{2}\right) \lambda_{\pi}\left(t_{1}\right)\right\rangle\right] .
$$

At order zero in perturbation theory, in terms of Feynman diagrams, Eq. (6.10) can be written as follows:

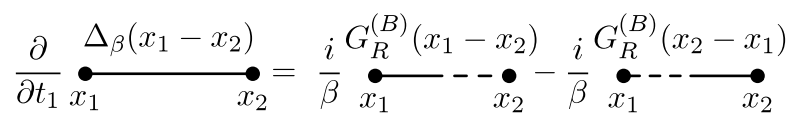

We will provide a proof of this in Appendix G.

At the first order in perturbation theory the diagrammatic form of the FDT (6.10) is:

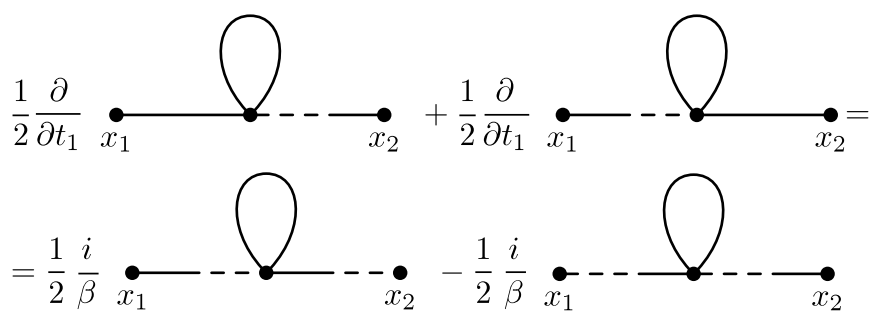

and also this will be proved in Appendix G.

The second order result is given by the following three diagrammatic relations.

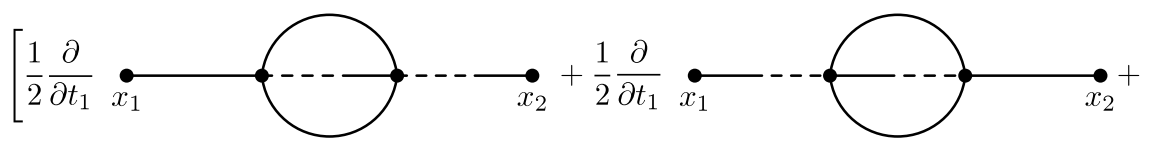



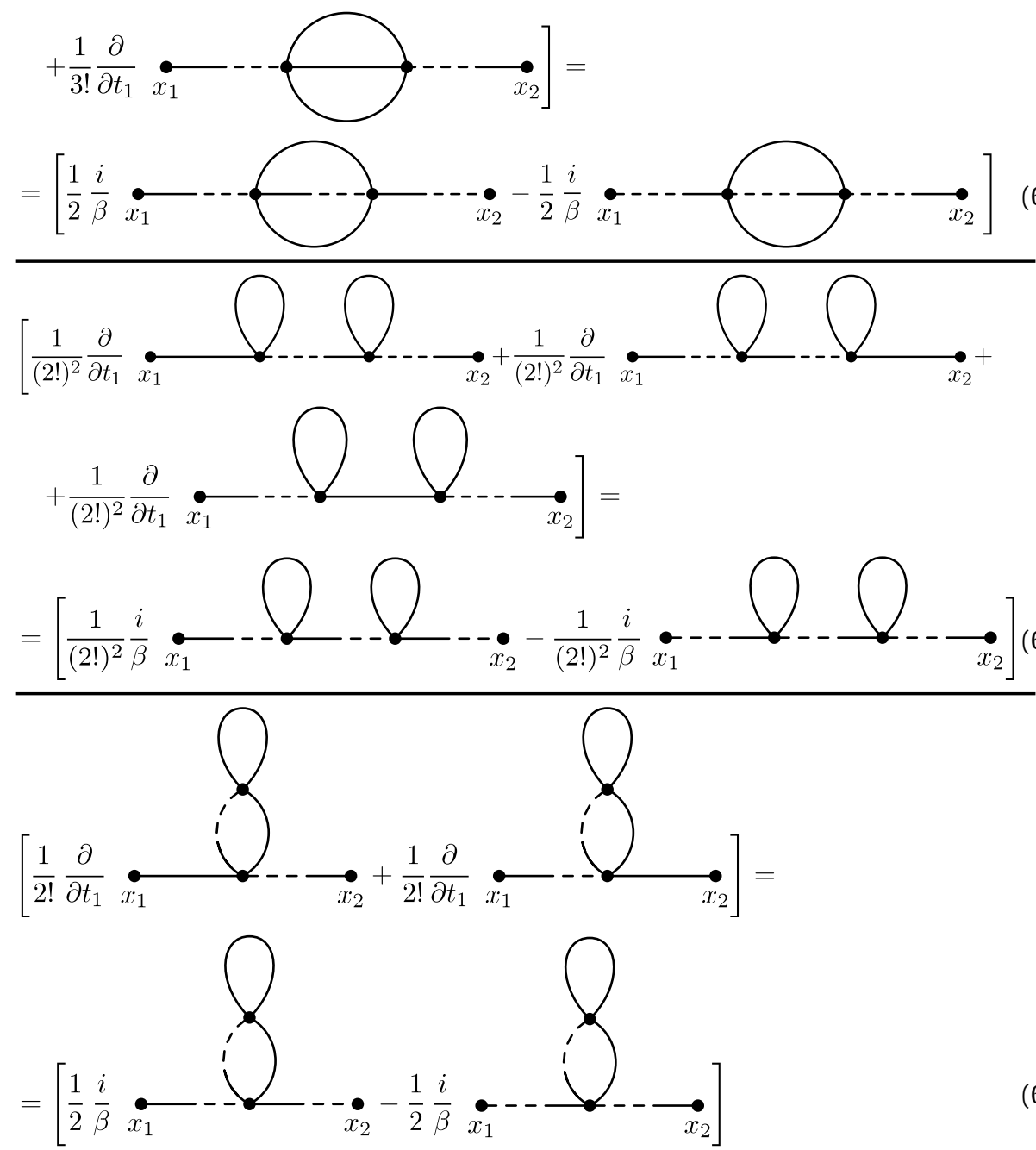

We feel that all these diagrammatic identities will turn out to be very useful in simplifying long sums of diagrams which may appear in phenomenological applications of our formalism.

\section{Conclusions and outlooks}

In this paper we have developed the Feynman diagrams for a classical $g \frac{\phi^{4}(x)}{4 !}$ field theory either with and without temperature. This topic is of high interest in the field of heavy ions scattering where classical field theory with temperature seems to be playing a central role. Besides this application, the Feynman diagrams for a classical theory can be useful in many fields like, for example, planetary motion where there is a vast literature of perturbative analytic calculations which could be replaced, and even speeded up, by some simple Feynman diagrams.

We have been able do develop the Feynman diagrams in a very easy way because a path-integral for classical systems had been previously developed [11]. With respect to other approaches to perturbation theory for classical systems, we have been able to give the Feynman diagrams not only for the Bosonic variables of the system and for the response fields, but also for the Jacobi fields associated to the dynamics. 
Besides developing this diagrammatics for all these fields and their interactions, we have showed that the many different classical diagrams of the various different fields mentioned above can be unified in few super-diagrams which have the same kind of vertices and kinetic term as the quantum one associated to $\varphi^{a}$.

We hope that this super-diagram formalism can be of some help not only in simplifying the notation and making feasible higher order analytic computations, but also in understanding the subtle interplay between the quantum high temperature behavior and the classical one. Interplay which is very important in the heavy ions scattering field. This field needs the development of a perturbative approach to real hot-classical-QCD. To do that, several preliminary problems need to be addressed. The first one would be the introduction of fermions and non-abelian gauge fields in this classical formalism. This first step has already been done: fermions were introduced in [35] and nonabelian gauge fields in [36]. The second step, perturbation theory, at least for classical YM, is under construction and it will be released in the future [37]. The third step is to develop a sort of effective action generated by the temperature effects on a classical field. This last step, at least for a scalar field, has already achieved some progress [38] and it will be released soon.

What has been done in this first paper is aimed at providing the formal tools which could later be used by physicists for applications closer to nature.

\section{Acknowledgments}

AFN acknowledges hospitality and financial support from ICTP and INFN in Trieste and financial support from CNPq-Brazil under the grant 307824/2009-8. The work of EG and EC has been supported by grants from the MIUR (Prin 2008) and INFN (IS GE41) of Italy. Last, but not least, we thank G. Aarts for helpful e-mail exchanges and R. Penco and M. Reuter for their suggestions, support and generosity.

\section{Appendix A. A fundamental identity}

In this appendix we will show the details of how to go from (4.13) to (4.15). If we insert (4.5) into (4.13) we get:

$$
\begin{aligned}
\tilde{\mathcal{Z}}_{\beta}\left[J_{\phi}\right]= & \int \mathscr{D} \phi_{i}(\vec{x}) \exp \left\{-\beta \int d^{3} \vec{x}\left[\frac{\left(\nabla \phi_{i}\right)^{2}}{2}+\frac{m^{2} \phi_{i}^{2}}{2}\right]\right. \\
& \left.+i \int d^{4} x J_{\phi}(x) \int d^{3} \vec{x}^{\prime} \phi_{i}\left(x^{\prime}\right) a_{\phi}\left(\vec{x}-\vec{x}^{\prime}, t\right)\right\} \\
& \times \int \mathscr{D} \pi_{i}(\vec{x}) \exp \left[-\beta \int d^{3} \vec{x} \frac{\pi_{i}^{2}(\vec{x})}{2}+i \int d^{4} x J_{\phi}(x) \int d^{3} \vec{x}^{\prime} \pi_{i}\left(\vec{x}^{\prime}\right) a_{\pi}\left(\vec{x}-\vec{x}^{\prime}, t\right)\right] .
\end{aligned}
$$

Let us now define the following quantities:

$$
\begin{aligned}
& J_{\phi}^{a}\left(\vec{x}^{\prime}\right)=i \int d t d^{3} \vec{x} J_{\phi}(\vec{x}, t) a_{\phi}\left(\vec{x}-\vec{x}^{\prime}, t\right), \\
& J_{\pi}^{a}\left(\vec{x}^{\prime}\right)=i \int d t d^{3} \vec{x} J_{\phi}(\vec{x}, t) a_{\pi}\left(\vec{x}-\vec{x}^{\prime}, t\right)
\end{aligned}
$$

and let us rewrite the normalized $\tilde{\mathcal{Z}}_{\beta}\left[J_{\phi}\right]$ using (A.2) and (A.3):

$$
\begin{aligned}
\tilde{\mathcal{Z}}_{\beta}\left[J_{\phi}\right]= & \frac{1}{\tilde{\mathcal{Z}}_{\beta}[0]^{\phi}} \int \mathscr{D} \phi_{i}(\vec{x}) \exp \left\{-\beta \int d^{3} \vec{x}\left[\frac{\left(\nabla \phi_{i}\right)^{2}}{2}+\frac{m^{2} \phi_{i}^{2}}{2}\right]+\int d^{3} \vec{x} \phi_{i}(\vec{x}) J_{\phi}^{a}(\vec{x})\right\} \\
& \times \frac{1}{\tilde{\mathcal{Z}}_{\beta}[0]^{\pi}} \int \mathscr{D} \pi_{i}(\vec{x}) \exp \left\{-\beta \int d^{3} \vec{x} \frac{\pi_{i}^{2}(\vec{x})}{2}+\int d^{3} \vec{x} \pi_{i}(\vec{x}) J_{\pi}^{a}(\vec{x})\right\},
\end{aligned}
$$


where

$$
\begin{aligned}
& \tilde{\mathcal{Z}}_{\beta}[0]^{\phi}=\int \mathscr{D} \phi_{i}(\vec{x}) \exp \left\{-\beta \int d^{3} \vec{x}\left[\frac{\left(\nabla \phi_{i}\right)^{2}}{2}+\frac{m^{2} \phi_{i}^{2}}{2}\right]\right\} \\
& \tilde{\mathcal{Z}}_{\beta}[0]^{\pi}=\int \mathscr{D} \pi_{i}(\vec{x}) \exp \left\{-\beta\left[\int d^{3} \vec{x} \frac{\pi_{i}^{2}(\vec{x})}{2}\right]\right\} .
\end{aligned}
$$

Performing a partial integration for the kinetic piece in Eq. (A.4) we get:

$$
\begin{aligned}
\int \mathscr{D} \phi_{i}(\vec{x}) \exp \left\{-\frac{1}{2} \int d^{3} \vec{x} d^{3} \vec{x}^{\prime} \phi_{i}(\vec{x})\left[\beta \delta\left(\vec{x}-\vec{x}^{\prime}\right)\left(-\nabla^{2^{\prime}}+m^{2}\right)\right] \phi_{i}\left(\vec{x}^{\prime}\right)\right. \\
\left.+\int d^{3} \vec{x} \phi_{i}(\vec{x}) J_{\phi}^{a}(\vec{x})\right\} .
\end{aligned}
$$

As the weight is quadratic, the integration can be formally done for all (A.4), and the numerator of (A.4) turns out to be:

$$
\exp \left[\frac{1}{2} \int d^{3} \vec{x} d^{3} \vec{x}^{\prime} J_{\phi}^{a}(\vec{x}) A^{-1}\left(\vec{x}, \vec{x}^{\prime}\right) J_{\phi}^{a}\left(\vec{x}^{\prime}\right)\right] \tilde{Z}_{\beta}[0]^{\phi}
$$

where $A\left(\vec{x}, \vec{x}^{\prime}\right)=\beta \delta\left(\vec{x}-\vec{x}^{\prime}\right)\left(-\nabla^{2^{\prime}}+m^{2}\right)$ and it obeys the equation:

$$
\begin{aligned}
\int d^{3} \vec{x}^{\prime} A\left(\vec{x}, \vec{x}^{\prime}\right) A^{-1}\left(\vec{x}^{\prime}, \vec{x}^{\prime \prime}\right) & =\delta\left(\vec{x}-\vec{x}^{\prime \prime}\right) \\
& =\int d^{3} \vec{x}^{\prime}\left[\beta \delta\left(\vec{x}-\vec{x}^{\prime}\right)\left(-\nabla^{2^{\prime}}+m^{2}\right)\right] A^{-1}\left(\vec{x}^{\prime}, \vec{x}^{\prime \prime}\right) \\
& =\delta\left(\vec{x}-\vec{x}^{\prime \prime}\right),
\end{aligned}
$$

so,

$$
\left[\beta\left(-\nabla^{2}+m^{2}\right) A^{-1}\right]\left(\vec{x}, \vec{x}^{\prime \prime}\right)=\delta\left(\vec{x}-\vec{x}^{\prime \prime}\right) .
$$

Performing the Fourier transform of $A^{-1}$ we get:

$$
A^{-1}\left(\vec{x}, \vec{x}^{\prime \prime}\right)=\frac{1}{(2 \pi)^{3}} \int d^{3} \vec{p} e^{-i \vec{p} \cdot\left(\vec{x}^{\prime \prime}-\vec{x}\right)} \tilde{A}^{-1}(\vec{p}) .
$$

It follows from (A.10) that

$$
\tilde{A}^{-1}(\vec{p})=\frac{1}{\beta\left(\vec{p}^{2}+m^{2}\right)} .
$$

Using this and the expressions (A.2), (A.3), (4.11) and (4.12) we obtain, for the argument in the exponent of (A.9), the following expression:

$$
\begin{aligned}
& \int d^{3} \vec{x} d^{3} \vec{x}^{\prime}\left\{i \int d^{3} \vec{x}_{1} d t_{1} J_{\phi}\left(\vec{x}_{1}, t_{1}\right) \int \frac{d^{3} \vec{p}_{1}}{(2 \pi)^{3}} e^{i \vec{p}_{1} \cdot\left(\vec{x}_{1}-\vec{x}\right)} \cos \left[E_{\vec{p}_{1}}\left(t_{1}-t_{i}\right)\right]\right\} \\
& \times\left[\int \frac{d^{3} \vec{p}}{(2 \pi)^{3}} e^{i \vec{p} \cdot\left(\vec{x}^{\prime}-\vec{x}\right)} \tilde{A}^{-1}(\vec{p})\right]\left\{i \int d^{3} \vec{x}_{2} d t_{2} J_{\phi}\left(\vec{x}_{2}, t_{2}\right) \int \frac{d^{3} \vec{p}_{1}}{(2 \pi)^{3}} e^{i \vec{p}_{2} \cdot\left(\vec{x}_{2}-\vec{x}^{\prime}\right)}\right. \\
& \left.\times \cos \left[E_{\vec{p}_{2}}\left(t_{2}-t_{i}\right)\right]\right\}
\end{aligned}
$$

where $t_{i}$ is the initial time appearing in (4.4). Performing in the expression above the integration over $\vec{x}$ and $\vec{x}^{\prime}$ we get:

$$
-\int d^{4} x_{1} d^{4} x_{2} J_{\phi}\left(x_{1}\right) J_{\phi}\left(x_{2}\right) \int \frac{d^{3} \vec{p}}{(2 \pi)^{3}} e^{-i \vec{p} \cdot\left(\vec{x}_{1}-\vec{x}_{2}\right)} \tilde{A}^{-1}(\vec{p}) \cos \left[E_{\vec{p}}\left(t_{1}-t_{i}\right)\right] \cos \left[E_{\vec{p}}\left(t_{2}-t_{i}\right)\right] .
$$


So, (A.4) becomes:

$$
\begin{aligned}
& \frac{1}{\tilde{z}_{\beta}[0]^{\phi}} \int \mathscr{D} \phi_{i}(\vec{x}) \exp \left\{-\beta \int d^{3} \vec{x}\left[\frac{\left(\nabla \phi_{i}\right)^{2}}{2}+\frac{m^{2} \phi_{i}^{2}}{2}\right]+\int d^{3} \vec{x} \phi_{i}(\vec{x}) J_{\phi}^{a}(\vec{x})\right\} \\
& =\exp \left\{-\frac{1}{2} \int d^{3} \vec{x} d^{3} \vec{x}^{\prime} J_{\phi}(\vec{x})\left\{\int \frac{d^{3} \vec{p}}{(2 \pi)^{3}} \tilde{A}^{-1}(\vec{p}) \cos \left[E_{\vec{p}}\left(t-t_{i}\right)\right]\right.\right. \\
& \left.\left.\times \cos \left[E_{\vec{p}}\left(t^{\prime}-t_{i}\right)\right] e^{i \vec{p} \cdot\left(\vec{x}^{\prime}-\vec{x}\right)}\right\} J_{\phi}\left(\vec{x}^{\prime}\right)\right\}
\end{aligned}
$$

Let us now do an analogue set of manipulations for (A.5)

$$
\begin{aligned}
& \frac{1}{\tilde{\tilde{z}}_{\beta}[0]^{\pi}} \int \mathscr{D} \pi_{i}(\vec{x}) \exp \left[-\beta \int d^{3} \vec{x} \frac{\pi_{i}^{2}(\vec{x})}{2}+\int d^{3} \vec{x} \pi_{i}(\vec{x}) J_{\pi}^{a}(\vec{x})\right] \\
& =\exp \left[\int d^{3} \vec{x} d^{3} \vec{x}^{\prime} J_{\pi}^{a}(\vec{x}) B^{-1}\left(\vec{x}, \vec{x}^{\prime}\right) J_{\pi}^{a}\left(\vec{x}^{\prime}\right)\right] \\
& =\exp \left\{\frac{1}{2 \beta} \int d^{3} \vec{x}\left[J_{\pi}^{a}(\vec{x})\right]^{2}\right\}
\end{aligned}
$$

where $B\left(\vec{x}, \vec{x}^{\prime}\right) \equiv \beta \delta\left(\vec{x}-\vec{x}^{\prime}\right)$. Using the expression (A.2), (A.3), (4.11) and (4.12), the exponent in (A.17) becomes:

$$
\begin{aligned}
& \int d^{3} \vec{x}\left[J_{\pi}^{a}(\vec{x})\right]^{2} \\
& =\int d^{3} \vec{x}\left[i \int d t_{1} d^{3} \vec{x}_{1} J_{\phi}\left(\vec{x}_{1}, t_{1}\right) a_{\pi}\left(\vec{x}_{1}-\vec{x}, t_{1}\right)\right]\left[i \int d t_{2} d^{3} \vec{x}_{2} J_{\phi}\left(\vec{x}_{2}, t_{2}\right) a_{\pi}\left(\vec{x}_{2}-\vec{x}, t_{2}\right)\right] \\
& =-\int d x_{1} d x_{2} J_{\phi}\left(x_{1}\right) J_{\phi}\left(x_{2}\right)\left\{\int \frac{d^{3} \vec{p}}{(2 \pi)^{3}} \frac{\sin \left[E_{\vec{p}}\left(t_{1}-t_{i}\right)\right] \sin \left[E_{\vec{p}}\left(t_{2}-t_{i}\right)\right]}{E_{\vec{p}}^{2}} e^{-i \vec{p} .\left(\vec{x}_{2}-\vec{x}_{1}\right)}\right\} .
\end{aligned}
$$

Inserting (A.18) and (A.16) in (A.4) and (A.5) we obtain:

$$
\tilde{Z}_{\beta}\left[J_{\phi}\right]=\exp \left\{-\frac{1}{2} \int d^{4} x d^{4} x^{\prime} J_{\phi}(x)\left\{\frac{d^{3} \vec{p}}{(2 \pi)^{3}} \frac{\cos \left[E_{\vec{p}}\left(t-t^{\prime}\right)\right]}{\beta\left(\vec{p}^{2}+m^{2}\right)} e^{i \vec{p} \cdot\left(\vec{x}-\vec{x}^{\prime}\right)}\right\} J_{\phi}\left(x^{\prime}\right)\right\} .
$$

Via the identity

$$
\int d p^{0} \frac{1}{\left|p^{0}\right|} e^{-i p^{0}\left(t-t^{\prime}\right)} \delta\left(p^{2}-m^{2}\right)=\frac{\cos \left[E_{\vec{p}}\left(t-t^{\prime}\right)\right]}{E_{\vec{p}}^{2}}
$$

we can rewrite $\tilde{Z}_{\beta}\left[J_{\phi}\right]$ as

$$
\tilde{Z}_{\beta}\left[J_{\phi}\right]=\exp \left[-\frac{1}{2} \int d^{4} x d^{4} x^{\prime} J_{\phi}(x) \Delta_{\beta}\left(x-x^{\prime}\right) J_{\phi}\left(x^{\prime}\right)\right]
$$

where

$$
\Delta_{\beta}(x)=\int \frac{d^{4} p}{(2 \pi)^{4}} \frac{2 \pi}{\beta\left|p^{0}\right|} \delta\left(p^{2}-m^{2}\right) e^{-i p . x} .
$$

This is what we have used in (4.15). 


\section{Appendix B. Field correlations via super-field projections}

In this appendix we will present some of the detailed calculations that we skipped in the main body of the paper. Here, in particular, we will report the calculations which support the results in (5.29) and (5.30). Let us start from (5.29):

$$
\int d \theta_{1} d \bar{\theta}_{1} d \theta_{2} d \bar{\theta}_{2}\left(\bar{\theta}_{1} \theta_{1}\right)\left(\bar{\theta}_{2} \theta_{2}\right)\left[\frac{1}{(2 !)^{2}} \underset{z_{1}}{\underset{z}{2}}\right.
$$

Using the fact that $g(z, z)=0$ and the rules of Grassmannian integrations, we get for the expression above:

$$
\begin{aligned}
& \int d \theta_{1} d \bar{\theta}_{1} d \theta_{2} d \bar{\theta}_{2}\left(\bar{\theta}_{1} \theta_{1}\right)\left(\bar{\theta}_{2} \theta_{2}\right) \int d^{4} x d \theta d \bar{\theta} \int d^{4} x^{\prime} d \theta^{\prime} d \bar{\theta}^{\prime}\left[\Delta_{\beta}\left(x_{1}-x\right)+G_{R}^{(B)}\left(x_{1}-x\right) \bar{\theta} \theta\right. \\
& \left.+G_{R}^{(B)}\left(x-x_{1}\right) \bar{\theta}_{1} \theta_{1}+G_{R}^{(F)}\left(x_{1}-x\right) \theta_{1} \bar{\theta}+G_{R}^{(F)}\left(x-x_{1}\right) \theta \bar{\theta}_{1}\right]\left[\Delta_{\beta}(x-x)\right]\left[\Delta_{\beta}\left(x-x^{\prime}\right)\right. \\
& \left.+G_{R}^{(B)}\left(x-x^{\prime}\right) \bar{\theta}^{\prime} \theta^{\prime}+G_{R}^{(B)}\left(x^{\prime}-x\right) \bar{\theta} \theta+G_{R}^{(F)}\left(x-x^{\prime}\right) \theta \bar{\theta}^{\prime}+G_{R}^{(F)}\left(x^{\prime}-x\right) \theta^{\prime} \bar{\theta}\right] \Delta_{\beta}\left(x^{\prime}-x^{\prime}\right) \\
& \times\left[\Delta_{\beta}\left(x^{\prime}-x_{2}\right)+G_{R}^{(B)}\left(x^{\prime}-x_{2}\right) \bar{\theta}_{2} \theta_{2}+G_{R}^{(B)}\left(x_{2}-x^{\prime}\right) \bar{\theta}^{\prime} \theta^{\prime}\right. \\
& \left.+G_{R}^{(F)}\left(x^{\prime}-x_{2}\right) \theta^{\prime} \bar{\theta}_{2}+G_{R}^{(F)}\left(x_{2}-x^{\prime}\right) \theta_{2} \bar{\theta}^{\prime}\right] \\
& =\frac{1}{4} \int d \theta_{1} d \bar{\theta}_{1} d \theta_{2} d \bar{\theta}_{2}\left(\bar{\theta}_{1} \theta_{1}\right)\left(\bar{\theta}_{2} \theta_{2}\right) \int d^{4} x d \theta d \bar{\theta} \int d^{4} x^{\prime} d \theta^{\prime} d \bar{\theta}^{\prime}\left[\Delta_{\beta}\left(x_{1}-x\right) \Delta_{\beta}\left(x^{\prime}-x_{2}\right)\right. \\
& +\Delta_{\beta}\left(x_{1}-x\right) G_{R}^{(B)}\left(x_{2}-x^{\prime}\right) \bar{\theta}^{\prime} \theta^{\prime}+G_{R}^{(B)}\left(x_{1}-x\right) \bar{\theta} \theta \Delta_{\beta}\left(x^{\prime}-x_{2}\right)+G_{R}^{(B)}\left(x_{1}-x\right) \bar{\theta} \theta \\
& \left.\times G_{R}^{(B)}\left(x_{2}-x^{\prime}\right) \bar{\theta}^{\prime} \theta^{\prime}\right] \Delta_{\beta}(x-x) \times\left[\Delta_{\beta}\left(x-x^{\prime}\right)+G_{R}^{(B)}\left(x-x^{\prime}\right) \bar{\theta}^{\prime} \theta^{\prime}+G_{R}^{(B)}\left(x^{\prime}-x\right) \bar{\theta} \theta\right. \\
& \left.+G_{R}^{(F)}\left(x-x^{\prime}\right) \theta \bar{\theta}^{\prime}+G_{R}^{(F)}\left(x^{\prime}-x\right) \theta^{\prime} \bar{\theta}\right] \Delta_{\beta}\left(x^{\prime}-x^{\prime}\right) \\
& =\frac{1}{4} \int d \theta_{1} d \bar{\theta}_{1} d \theta_{2} d \bar{\theta}_{2}\left(\bar{\theta}_{1} \theta_{1}\right)\left(\bar{\theta}_{2} \theta_{2}\right) \int d^{4} x d \theta d \bar{\theta} \int d^{4} x^{\prime} d \theta^{\prime} d \bar{\theta}^{\prime} \\
& \times\left\{\Delta_{\beta}\left(x_{1}-x\right) \Delta_{\beta}\left(x^{\prime}-x_{2}\right) \Delta_{\beta}(x-x)\left[\Delta_{\beta}\left(x-x^{\prime}\right)+g\left(z, z^{\prime}\right)\right] \Delta_{\beta}\left(x^{\prime}-x^{\prime}\right)\right. \\
& +\left[\Delta_{\beta}\left(x_{1}-x\right) G_{R}^{(B)}\left(x_{2}-x^{\prime}\right) \bar{\theta}^{\prime} \theta^{\prime} G_{R}^{(B)}\left(x^{\prime}-x\right) \bar{\theta} \theta\right. \\
& +G_{R}^{(B)}\left(x_{1}-x\right) \bar{\theta} \theta \Delta_{\beta}\left(x^{\prime}-x_{2}\right) G_{R}^{(B)}\left(x-x^{\prime}\right) \bar{\theta}^{\prime} \theta^{\prime} \\
& \left.\left.+G_{R}^{(B)}\left(x_{1}-x\right) \bar{\theta} \theta G_{R}^{(B)}\left(x_{2}-x^{\prime}\right) \bar{\theta}^{\prime} \theta^{\prime} \Delta_{\beta}\left(x-x^{\prime}\right)\right] \Delta_{\beta}(x-x) \Delta_{\beta}\left(x^{\prime}-x^{\prime}\right)\right\} \\
& =\frac{1}{4} \int d^{4} x \int d^{4} x^{\prime} \Delta_{\beta}\left(x_{1}-x\right) \Delta_{\beta}(x-x) G_{R}^{(B)}\left(x^{\prime}-x\right) \Delta_{\beta}\left(x^{\prime}-x^{\prime}\right) G_{R}^{(B)}\left(x_{2}-x^{\prime}\right) \\
& +\frac{1}{4} \int d^{4} x \int d^{4} x^{\prime} \Delta_{\beta}\left(x_{2}-x\right) \Delta_{\beta}(x-x) G_{R}^{(B)}\left(x^{\prime}-x\right) \Delta_{\beta}\left(x^{\prime}-x^{\prime}\right) G_{R}^{(B)}\left(x_{1}-x^{\prime}\right) \\
& +\frac{1}{4} \int d^{4} x \int d^{4} x^{\prime} G_{R}^{(B)}\left(x_{1}-x\right) \Delta_{\beta}(x-x) \Delta_{\beta}\left(x-x^{\prime}\right) \Delta_{\beta}\left(x^{\prime}-x^{\prime}\right) G_{R}^{(B)}\left(x_{2}-x^{\prime}\right) .
\end{aligned}
$$

The three diagrams presented in the last expression are precisely

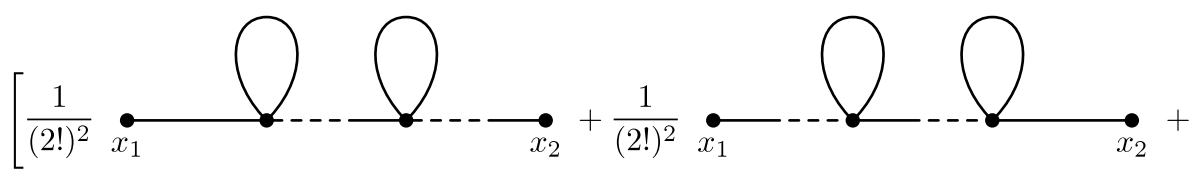




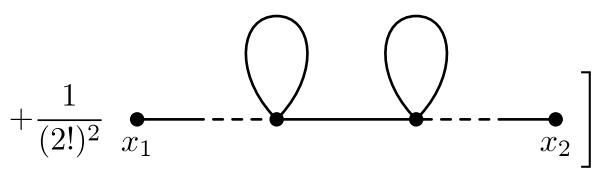

which is exactly the result we claimed in (5.29).

Let us now give the proof of (5.30). Again we will make use of $g(z, z)=0$ and of the standard rules of integration over Grassmannian coordinates.

$$
\begin{aligned}
& \int d \theta_{1} d \bar{\theta}_{1} d \theta_{2} d \bar{\theta}_{2}\left(\bar{\theta}_{1} \theta_{1}\right)\left(\bar{\theta}_{2} \theta_{2}\right)\left[\frac{1}{(2 !)^{2}} \underset{z_{1}}{\stackrel{(}{\rightleftharpoons}}\right. \\
& =\frac{1}{4} \int d \theta_{1} d \bar{\theta}_{1} d \theta_{2} d \bar{\theta}_{2}\left(\bar{\theta}_{1} \theta_{1}\right)\left(\bar{\theta}_{2} \theta_{2}\right) \int d^{4} x d \theta d \bar{\theta} \int d^{4} x^{\prime} d \theta^{\prime} d \bar{\theta}^{\prime}\left[\Delta_{\beta}\left(x_{1}-x\right)+G_{R}^{(B)}\left(x_{1}-x\right) \bar{\theta} \theta\right. \\
& \left.+G_{R}^{(B)}\left(x-x_{1}\right) \bar{\theta}_{1} \theta_{1}+G_{R}^{(F)}\left(x_{1}-x\right) \theta_{1} \bar{\theta}+G_{R}^{(F)}\left(x-x_{1}\right) \theta \bar{\theta}_{1}\right] \\
& \times\left[\Delta_{\beta}\left(x-x^{\prime}\right)+g\left(z, z^{\prime}\right)\right]\left[\Delta_{\beta}\left(x^{\prime}-x^{\prime}\right)+g\left(z^{\prime}, z^{\prime}\right)\right]\left[\Delta_{\beta}\left(x^{\prime}-x\right)+g\left(z^{\prime}, z\right)\right] \\
& \times\left[\Delta_{\beta}\left(x-x_{2}\right)+G_{R}^{(B)}\left(x-x_{2}\right) \bar{\theta}_{2} \theta_{2}+G_{R}^{(B)}\left(x_{2}-x\right) \bar{\theta} \theta\right. \\
& \left.+G_{R}^{(F)}\left(x-x_{2}\right) \theta \bar{\theta}_{2}+G_{R}^{(F)}\left(x_{2}-x\right) \theta_{2} \bar{\theta}\right] \\
& =\frac{1}{4} \int d \theta_{1} d \bar{\theta}_{1} d \theta_{2} d \bar{\theta}_{2}\left(\bar{\theta}_{1} \theta_{1}\right)\left(\bar{\theta}_{2} \theta_{2}\right) \int d^{4} x d \theta d \bar{\theta} \int d^{4} x^{\prime} d \theta^{\prime} d \bar{\theta}^{\prime} \\
& \times\left[\Delta_{\beta}\left(x_{1}-x\right) \Delta_{\beta}\left(x-x_{2}\right)+\Delta_{\beta}\left(x_{1}-x\right) G_{R}^{(B)}\left(x_{2}-x\right) \bar{\theta} \theta+G_{R}^{(B)}\left(x_{1}-x\right) \bar{\theta} \theta \Delta_{\beta}\left(x-x_{2}\right)\right. \\
& \left.+G_{R}^{(B)}\left(x_{1}-x\right) \bar{\theta} \theta G_{R}^{(B)}\left(x_{2}-x\right) \bar{\theta} \theta\right]\left[\Delta_{\beta}\left(x-x^{\prime}\right)+g\left(z, z^{\prime}\right)\right] \\
& \times \Delta_{\beta}\left(x^{\prime}-x^{\prime}\right)\left[\Delta_{\beta}\left(x^{\prime}-x\right)+g\left(z^{\prime}, z\right)\right] \\
& =\frac{1}{4} \int d \theta_{1} d \bar{\theta}_{1} d \theta_{2} d \bar{\theta}_{2}\left(\bar{\theta}_{1} \theta_{1}\right)\left(\bar{\theta}_{2} \theta_{2}\right) \int d^{4} x d \theta d \bar{\theta} \int d^{4} x^{\prime} d \theta^{\prime} d \bar{\theta}^{\prime} \\
& \times\left\{\left[\Delta_{\beta}\left(x_{1}-x\right) G_{R}^{(B)}\left(x_{2}-x\right) \bar{\theta} \theta\right]\left[2 \Delta_{\beta}\left(x-x^{\prime}\right) G_{R}^{(B)}\left(x-x^{\prime}\right) \bar{\theta}^{\prime} \theta^{\prime}\right] \Delta_{\beta}\left(x^{\prime}-x^{\prime}\right)\right. \\
& \left.+\left[G_{R}^{(B)}\left(x_{1}-x\right) \bar{\theta} \theta \Delta_{\beta}\left(x-x_{2}\right)\right]\left[2 \Delta_{\beta}\left(x-x^{\prime}\right) G_{R}^{(B)}\left(x-x^{\prime}\right) \bar{\theta}^{\prime} \theta^{\prime}\right] \Delta_{\beta}\left(x^{\prime}-x^{\prime}\right)\right\} \\
& =\frac{1}{2} \int d^{4} x d^{4} x^{\prime} \Delta_{\beta}\left(x_{1}-x\right) G_{R}^{(B)}\left(x-x^{\prime}\right) \Delta_{\beta}\left(x^{\prime}-x^{\prime}\right) \Delta_{\beta}\left(x^{\prime}-x\right) G_{R}^{(B)}\left(x_{2}-x\right) \\
& +\frac{1}{2} \int d^{4} x d^{4} x^{\prime} G_{R}^{(B)}\left(x_{1}-x\right) G_{R}^{(B)}\left(x-x^{\prime}\right) \Delta_{\beta}\left(x^{\prime}-x^{\prime}\right) \Delta_{\beta}\left(x^{\prime}-x\right) \Delta_{\beta}\left(x-x_{2}\right)
\end{aligned}
$$

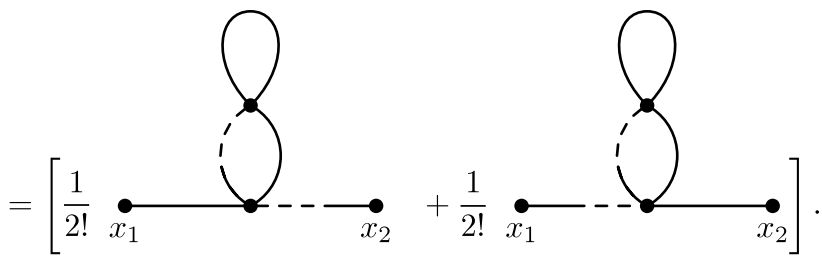

This proves (5.30). 
In both of these proofs we have been rather pedantic with the details of the calculations. We did that because we wanted to help the reader not familiar with our formalism in going through the details of the calculations.

\section{Appendix C. Loop identities}

In this appendix we will prove various identities which, among other things, will help us in "proving" Eq. (5.47). We have put quotation marks around "proving", because ours will not be an analytic proof. Anyhow the identities that we will get will turn out to be rather useful. Let us use the following notation: $d \mu \equiv d \theta d \bar{\theta}, d \mu_{1} \equiv d \theta_{1} d \bar{\theta}_{1}$, etc. The first identity we want to prove is:

$$
\int d \mu d \mu_{1} \ldots d \mu_{n} \mathbb{G}\left(z, z_{1}\right) \mathbb{G}\left(z_{1}, z_{2}\right) \ldots \mathbb{G}\left(z_{n}, z\right)=0 .
$$

We shall call it the "first loop identity", because the strings of $\mathbb{G}$ makes a super-loop. Let us first observe that

$$
\begin{aligned}
\int d z \mathbb{G}\left(z_{1}, z\right) \mathbb{G}\left(z, z_{2}\right)= & \int d^{4} x\left[G_{R}^{(B)}\left(x_{1}-x\right) \Delta_{\beta}\left(x-x_{2}\right)+G_{R}^{(B)}\left(x_{2}-x\right) \Delta_{\beta}\left(x_{1}-x\right)\right] \\
& +\int d^{4} x\left[G_{R}^{(B)}\left(x_{1}-x\right) G_{R}^{(B)}\left(x-x_{2}\right)\right] \bar{\theta}_{2} \theta_{2} \\
& +\int d^{4} x\left[G_{R}^{(B)}\left(x_{2}-x\right) G_{R}^{(B)}\left(x-x_{1}\right)\right] \bar{\theta}_{1} \theta_{1} \\
& +\int d^{4} x\left[G_{R}^{(F)}\left(x_{1}-x\right) G_{R}^{(F)}\left(x-x_{2}\right)\right] \theta_{1} \bar{\theta}_{2} \\
& +\int d^{4} x\left[G_{R}^{(F)}\left(x_{2}-x\right) G_{R}^{(F)}\left(x-x_{1}\right)\right] \theta_{2} \bar{\theta}_{1} .
\end{aligned}
$$

If we add to the l.h.s. of Eq. (C.2) one more $\mathbb{G}$ and build the expression

$$
\int d z d z^{\prime} \mathbb{G}\left(z_{1}, z\right) \mathbb{G}\left(z, z^{\prime}\right) \mathbb{G}\left(z^{\prime}, z_{2}\right),
$$

we can then do the integration over the Grassmannian variables and easily prove that:

$$
\begin{aligned}
& \int d z d z^{\prime} \mathbb{G}\left(z_{1}, z\right) \mathbb{G}\left(z, z^{\prime}\right) \mathbb{G}\left(z^{\prime}, z_{2}\right)=\int d^{4} x\left[G_{R}^{(B)}\left(x_{1}-x\right) \Delta_{\beta}\left(x-x^{\prime}\right) G_{R}^{(B)}\left(x_{2}-x^{\prime}\right)\right. \\
& +\Delta_{\beta}\left(x_{1}-x\right) G_{R}^{(B)}\left(x^{\prime}-x\right) G_{R}^{(B)}\left(x_{2}-x^{\prime}\right) \\
& \left.+G_{R}^{(B)}\left(x_{1}-x\right) G_{R}^{(B)}\left(x-x^{\prime}\right) \Delta_{\beta}\left(x^{\prime}-x_{2}\right)\right] \\
& +\int d^{4} x\left[G_{R}^{(B)}\left(x_{1}-x\right) G_{R}^{(B)}\left(x-x^{\prime}\right) G_{R}^{(B)}\left(x^{\prime}-x_{2}\right)\right] \bar{\theta}_{2} \theta_{2} \\
& +\int d^{4} x\left[G_{R}^{(B)}\left(x_{2}-x^{\prime}\right) G_{R}^{(B)}\left(x^{\prime}-x\right) G_{R}^{(B)}\left(x-x_{1}\right)\right] \bar{\theta}_{1} \theta_{1} \\
& +\int d^{4} x\left[G_{R}^{(F)}\left(x_{1}-x\right) G_{R}^{(F)}\left(x-x^{\prime}\right) G_{R}^{(F)}\left(x^{\prime}-x_{2}\right)\right] \theta_{1} \bar{\theta}_{2} \\
& +\int d^{4} x\left[G_{R}^{(F)}\left(x_{2}-x^{\prime}\right) G_{R}^{(F)}\left(x^{\prime}-x\right) G_{R}^{(F)}\left(x-x_{1}\right)\right] \theta_{2} \bar{\theta}_{1} .
\end{aligned}
$$

In general, with a string of all $\mathbb{G}$, we can prove by induction that the following relation holds (where $\left.z \equiv z_{0}, z^{\prime} \equiv z_{n+1}\right)$ :

$$
\int d z_{1} d z_{2} \ldots d z_{n} \mathbb{G}\left(z, z_{1}\right) \mathbb{G}\left(z_{1}, z_{2}\right) \ldots \mathbb{G}\left(z_{n}, z^{\prime}\right)=\left(G_{R}^{(B)} \star \cdots \star \Delta_{\beta i, i+1} \star \cdots \star G_{R}^{(B)}\right)\left(x_{0}, x_{n+1}\right)
$$




$$
\begin{aligned}
& +\left(G_{R}^{(B)} * \cdots * G_{R}^{(B)}\right)\left(x_{0}, x_{n+1}\right) \bar{\theta}_{n+1} \theta_{n+1}+\left(G_{R}^{(B)} * \cdots * G_{R}^{(B)}\right)\left(x_{n+1}, x_{0}\right) \bar{\theta}_{0} \theta_{0} \\
& +\left(G_{R}^{(F)} * \cdots * G_{R}^{(F)}\right)\left(x_{0}, x_{n+1}\right) \theta_{0} \bar{\theta}_{n+1}+\left(G_{R}^{(F)} * \cdots * G_{R}^{(F)}\right)\left(x_{n+1}, x_{0}\right) \theta_{n+1} \bar{\theta}_{0} .
\end{aligned}
$$

The various symbols appearing above are defined as follows:

$$
\begin{aligned}
& \left(G_{R}^{(B)} * \cdots * G_{R}^{(B)}\right)\left(x_{0}, x_{n+1}\right) \\
& =\int d^{4} x_{1} \ldots d^{4} x_{n} G_{R}^{(B)}\left(x_{0}-x_{1}\right) G_{R}^{(B)}\left(x_{1}-x_{2}\right) \ldots G_{R}^{(B)}\left(x_{n}-x_{n+1}\right) .
\end{aligned}
$$

(we call this "convolution".) While the first expression on the r.h.s. of Eq. (C.5) is defined as:

$$
\begin{aligned}
& \left(G_{R}^{(B)} \star \cdots \star \Delta_{\beta i, i+1} \star \cdots \star G_{R}^{(B)}\right)\left(x_{0}, x_{n+1}\right) \\
& \equiv \int d^{4} x_{1} \ldots d^{4} x_{n} G_{R}^{(B)}\left(x_{0}-x_{1}\right) G_{R}^{(B)}\left(x_{1}-x_{2}\right) \ldots G_{R}^{(B)}\left(x_{i-2}-x_{i-1}\right) G_{R}^{(B)}\left(x_{i-1}-x_{i}\right) \\
& \quad \times \Delta_{\beta}\left(x_{i}-x_{i+1}\right) G_{R}^{(B)}\left(x_{i+2}-x_{i+1}\right) G_{R}^{(B)}\left(x_{i+3}-x_{i+2}\right) \ldots G_{R}^{(B)}\left(x_{n}-x_{n+1}\right)
\end{aligned}
$$

(we call this "oriented convolution"). Eq. (C.5) can be proved by induction as follows. Let us take $P(n)=$ (Proposition of order $n$ ) to be given by Eq. (C.5). We already know, from the beginning of this appendix, that $P(1)$ holds true [see Eq. (C.2)]. Next, we need to show that $P(n) \Rightarrow P(n+1)$. Indeed, take $z^{\prime} \equiv z_{n+1}$ in Eq. (C.5), multiply it by $\mathbb{G}\left(z_{n+1}, z^{\prime}\right)$ and integrate over $z_{n+1}$. Using the definitions in Eqs. (C.6) and (C.7) the result follows in a straightforward manner. Let bus now go back to (C.5), take $z=z^{\prime}$ and integrate over $z$, using the definitions (C.6) and (C.7), we easily get:

$$
\int d z d z_{1} \ldots d z_{n} \mathbb{G}\left(z, z_{1}\right) \mathbb{G}\left(z_{1}, z_{2}\right) \ldots \mathbb{G}\left(z_{n}, z\right)=0 .
$$

A second loop equality which can be proved is

$$
\int d \mu d \mu_{1} \ldots d \mu_{n} \mathbb{G}^{m}\left(z, z_{1}\right) \mathbb{G}^{m_{1}}\left(z_{1}, z_{2}\right) \ldots \mathbb{G}^{m_{n}}\left(z_{n}, z\right)=0 .
$$

This is a generalization of (C.8) which is recovered when $m=m_{1}=\cdots=m_{n}=1$. Using the property (4) of Eq. (5.17) we get that the l.h.s. of Eq. (C.9) is equal to:

$$
\begin{aligned}
\int & d \mu d \mu_{1} \ldots d \mu_{n} \mathbb{G}^{m}\left(z, z_{1}\right) \mathbb{G}^{m_{1}}\left(z_{1}, z_{2}\right) \ldots \mathbb{G}^{m_{n}}\left(z_{n}, z\right) \\
= & \int d \mu d \mu_{1} \ldots d \mu_{n}\left[\Delta_{\beta}^{m}\left(x-x_{1}\right)+m \Delta_{\beta}^{m-1}\left(x-x_{1}\right) g\left(z, z_{1}\right)\right] \\
& \times\left[\Delta_{\beta}^{m_{1}}\left(x_{1}-x_{2}\right)+m_{1} \Delta_{\beta}^{m_{1}-1}\left(x_{1}-x_{2}\right) g\left(z_{1}, z_{2}\right)\right] \\
& \times \cdots \times\left[\Delta_{\beta}^{m_{n}}\left(x_{n}-x\right)+m_{n} \Delta_{\beta}^{m_{n}-1}\left(x_{n}-x\right) g\left(z_{n}, z\right)\right] \\
= & m m_{1} \ldots m_{n} \Delta_{\beta}^{m-1}\left(x-x_{1}\right) \Delta_{\beta}^{m_{1}-1}\left(x_{1}-x_{2}\right) \ldots \Delta_{\beta}^{m_{n}-1}\left(x_{n}-x\right) \\
& \times \int d \mu d \mu_{1} \ldots d \mu_{n}\left[g\left(z, z_{1}\right) g\left(z_{1}, z_{2}\right) \ldots g\left(z_{n}, z\right)\right]=0 .
\end{aligned}
$$

In the steps above we have made use of (C.8), and of the relations:

$$
\begin{aligned}
& \int d \mu d \mu_{1} \ldots d \mu_{n} g\left(z, z_{1}\right) g\left(z_{1}, z_{2}\right) \ldots g\left(z_{i-1}, z_{i}\right) \\
& \quad \times \Delta_{\beta}^{m_{i}}\left(x_{i}-x_{i+1}\right) g\left(z_{i+1}, z_{i+2}\right) \ldots g\left(z_{n}, z\right)=0
\end{aligned}
$$


and

$$
\int d \mu d \mu_{1} \ldots d \mu_{n} \Delta_{\beta}^{m}\left(z, z_{1}\right) \Delta_{\beta}^{m_{1}}\left(z_{1}, z_{2}\right) \ldots \Delta_{\beta}^{m_{n}}\left(z_{n}, z\right)=0 .
$$

Relations (C.11) and (C.12) can be easily proved considering the mismatch in the number of integration variables with respect to the variables present in the argument.

We should not forget that we have to prove relation $(5.47)$. The $\mathbb{F}^{(n)}\left(z, z^{\prime}\right)$ will be any combination of two-point functions with any number of loops inserted in order to reach the $n$-th order in perturbation theory.

Let us start from $n=1$ which is:

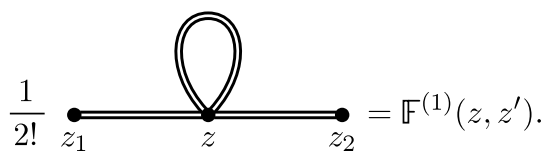

It is easy to prove (5.47) for this $\mathbb{F}^{(1)}$, in fact:

$$
\begin{aligned}
& \int d \theta_{1} d \theta_{2} d \bar{\theta}_{1} d \bar{\theta}_{2} \underset{z_{1}}{\longrightarrow} z_{2}=-\bullet x_{1} \\
& =-\int d z G_{R}^{(B)}\left(x-x_{1}\right) \Delta_{\beta}(x-x) G_{R}^{(B)}\left(x-x_{2}\right)=0
\end{aligned}
$$

This result comes from the fact that the integration contains $d \theta d \bar{\theta}$, but the integrand does not contain any $\theta, \bar{\theta}$.

Let us pass to the $\mathbb{F}^{(2)}\left(z, z^{\prime}\right)$ functions. We could choose, for example, the following one:

$$
\begin{aligned}
\int & d \theta_{1} d \theta_{2} d \bar{\theta}_{1} d \bar{\theta}_{2} \\
= & -\int d z d z^{\prime} G_{R}^{(B)}\left(x-x_{1}\right)\left[\mathbb{G}\left(z, z^{\prime}\right)\right]^{3} G_{R}^{(B)}\left(x^{\prime}-x_{2}\right) \\
= & -\int d^{4} x d^{4} x^{\prime} G_{R}^{(B)}\left(x-x_{1}\right) G_{R}^{(B)}\left(x^{\prime}-x_{2}\right)\left[\int d \mu d \mu^{\prime} \mathbb{G}^{3}\left(z, z^{\prime}\right)\right] \\
= & -\int d^{4} x d^{4} x^{\prime} G_{R}^{(B)}\left(x-x_{1}\right) G_{R}^{(B)}\left(x^{\prime}-x_{2}\right)\left[\int d \mu d \mu^{\prime} \mathbb{G}^{2}\left(z, z^{\prime}\right) \mathbb{G}\left(z^{\prime}, z\right)\right] .
\end{aligned}
$$

In the last step we have used property (1) of Eq. (5.14). The piece in the last equality of Eq. (C.15) contained in square brackets is zero because of the loop identity in Eq. (C.8). So, everything turns out to be zero. Another example of the $\mathbb{F}^{(2)}\left(z, z^{\prime}\right)$ function is

$$
\begin{aligned}
& \int d \theta_{1} d \theta_{2} d \bar{\theta}_{1} d \bar{\theta}_{2} \\
& =-\int d z d z^{\prime} G_{R}^{(B)}\left(x-x_{1}\right) \mathbb{G}\left(z, z^{\prime}\right) \mathbb{G}\left(z^{\prime}, z^{\prime}\right) \mathbb{G}\left(z^{\prime}, z\right) G_{R}^{(B)}\left(x-x_{2}\right) \\
& =-\int d^{4} x d^{4} x^{\prime} G_{R}^{(B)}\left(x-x_{1}\right) G_{R}^{(B)}\left(x^{\prime}-x_{2}\right)\left[\int d \mu d \mu^{\prime} \mathbb{G}\left(z, z^{\prime}\right) \mathbb{G}\left(z^{\prime}, z^{\prime}\right) \mathbb{G}\left(z^{\prime}, z\right)\right] .
\end{aligned}
$$


Once again the piece above contained in square brackets is zero because of the loop identity of Eq. (C.8).

We checked many two-point functions of the third order in perturbation theory and we got zero because of the loop identity (C.8). We do not have an analytical proof to all orders, but we believe, from the many examples we worked out, that (5.47) is indeed true.

\section{Appendix D. Jacobi field correlations}

In this appendix we will prove, to all orders in perturbation theory, that:

$$
\left\langle c^{\phi}\left(x_{1}\right) c^{\phi}\left(x_{2}\right)\right\rangle_{\beta}=0 .
$$

The reader could think that in $\tilde{\mathscr{L}}$ the $c^{\phi}$ and $c^{\phi}$ never interact and this could be the real reason behind (D.1). But, actually, the $c^{\phi}$ interact with $\phi$ and so, through the $\phi$, they could interact among themselves.

We know from (5.38) that

$$
\int d \theta_{1} d \theta_{2} d \bar{\theta}_{1} d \bar{\theta}_{2} \bar{\theta}_{2} \bar{\theta}_{1}\left\langle\Phi\left(x_{1}\right) \Phi\left(x_{2}\right)\right\rangle_{\beta}=\left\langle c^{\phi}\left(x_{1}\right) c^{\phi}\left(x_{2}\right)\right\rangle_{\beta} .
$$

The l.h.s at the $n$-th order of perturbation theory will have the general form:

$$
\int d \theta_{1} d \theta_{2} d \bar{\theta}_{1} d \bar{\theta}_{2} \bar{\theta}_{2} \bar{\theta}_{1} \int d z d z^{\prime} \mathbb{G}\left(z_{1}, z\right) \mathbb{F}^{(n)}\left(z, z^{\prime}\right) \mathbb{G}\left(z^{\prime}, z_{2}\right)
$$

where $\mathbb{F}^{(n)}$ is a function $\mathcal{O}\left(g^{n}\right)$ that we do not write down explicitly. For the first and second orders its explicit form is

$$
\begin{aligned}
& \mathbb{F}^{(1)}=\frac{1}{2} \mathbb{G}(z, z) \delta\left(z-z^{\prime}\right) \\
& \mathbb{F}^{(2)}=\left\{\begin{array}{l}
\frac{1}{6} \mathbb{G}^{3}\left(z, z^{\prime}\right) \\
\frac{1}{4} \mathbb{G}(z, z) \mathbb{G}\left(z, z^{\prime}\right) \mathbb{G}\left(z^{\prime}, z^{\prime}\right) \\
\frac{1}{4} \int d z^{\prime \prime} \mathbb{G}\left(z, z^{\prime \prime}\right) \mathbb{G}\left(z^{\prime \prime}, z^{\prime \prime}\right) \mathbb{G}\left(z^{\prime \prime}, z\right) \delta\left(z-z^{\prime}\right) .
\end{array}\right.
\end{aligned}
$$

The explicit form of (D.3) turns out to be:

$$
\begin{aligned}
(\mathrm{D} .3)= & \int d \theta_{1} d \theta_{2} d \bar{\theta}_{1} d \bar{\theta}_{2} \bar{\theta}_{2} \bar{\theta}_{1} \int d^{4} x d \theta d \bar{\theta} \int d^{4} x^{\prime} d \theta^{\prime} d \bar{\theta}^{\prime} \\
& \times\left[\Delta_{\beta}\left(x_{1}-x\right)+G_{R}^{(B)}\left(x_{1}-x\right) \bar{\theta} \theta+G_{R}^{(B)}\left(x-x_{1}\right) \bar{\theta}_{1} \theta_{1}\right. \\
& \left.+G_{R}^{(F)}\left(x_{1}-x\right) \theta_{1} \bar{\theta}+G_{R}^{(F)}\left(x-x_{1}\right) \theta \bar{\theta}_{1}\right] \mathbb{F}^{(n)}\left(z, z^{\prime}\right) \\
& \times\left[\Delta_{\beta}\left(x^{\prime}-x_{2}\right)+G_{R}^{(B)}\left(x^{\prime}-x_{2}\right) \bar{\theta}_{2} \theta_{2}+G_{R}^{(B)}\left(x_{2}-x^{\prime}\right) \bar{\theta}^{\prime} \theta^{\prime}\right. \\
& \left.+G_{R}^{(F)}\left(x^{\prime}-x_{2}\right) \theta^{\prime} \bar{\theta}_{2}+G_{R}^{(F)}\left(x_{2}-x^{\prime}\right) \theta_{2} \bar{\theta}^{\prime}\right] \\
= & \int d z d z^{\prime} \int d \theta_{1} d \theta_{2} d \bar{\theta}_{1} d \bar{\theta}_{2} \bar{\theta}_{2} \bar{\theta}_{1}\left[G_{R}^{(F)}\left(x_{1}-x\right) \theta_{1} \bar{\theta}\right] \mathbb{F}^{(n)}\left(z, z^{\prime}\right)\left[G_{R}^{(F)}\left(x_{2}-x^{\prime}\right) \theta_{2} \bar{\theta}^{\prime}\right] \\
= & \int d z d z^{\prime} \bar{\theta} \bar{\theta}^{\prime} G_{R}^{(F)}\left(x_{1}-x\right) \mathbb{F}^{(n)}\left(z, z^{\prime}\right) G_{R}^{(F)}\left(x_{2}-x^{\prime}\right) .
\end{aligned}
$$

The last integral above is zero. The reason is the following: $\mathbb{F}^{(n)}$ comes from the integration of products of various $G_{R}$. As each $G_{R}$ comes with an even number of $\hat{\theta}$ (where $\hat{\theta}$ indicates either $\theta$ or 
$\bar{\theta}$ ) attached, also $\mathbb{F}^{(n)}$ will have an even number of $\hat{\theta}$ because the integrations take away always an even number of $\hat{\theta}$. As $\mathbb{F}^{(n)}$ depends only on $z$ and $z^{\prime}$ and has an even number of $\hat{\theta}$, the only possible contributions must have the following strings of $\hat{\theta}$ :

(1) $\theta \bar{\theta} \theta^{\prime} \bar{\theta}^{\prime}$ which gives zero because $\theta^{\prime}$ is attached also to the final $G_{R}^{(B)}$ in the second equality in (D.6).

(2) $\theta \bar{\theta}$ which gives zero because of the initial $G_{R}^{(B)}$ (the term $\bar{\theta}_{1} \theta$ in $G_{R}^{(B)}$ gives zero because of the projector $\bar{\theta}_{1} \theta_{2}$ and the same for $\theta_{2}$ ).

(3) $\theta^{\prime} \bar{\theta}^{\prime}$ same as in (2) but because of the final $G_{R}^{(B)}$.

(4) $\theta \bar{\theta}^{\prime}$ same as in (3).

(5) $\bar{\theta} \theta^{\prime}$ same as in (2).

\section{Appendix E. Third order results for the two-point correlation}

In this appendix we will provide the third order perturbative calculations for the $\left\langle\phi\left(x_{1}\right) \phi\left(x_{2}\right)\right\rangle_{\beta}$ correlation using the super-field technique. We will list below the super-diagrams that are needed together with the corresponding symmetry factors. These factors were calculated using the algebraic rules given in [39]. The set of super-diagrams needed are
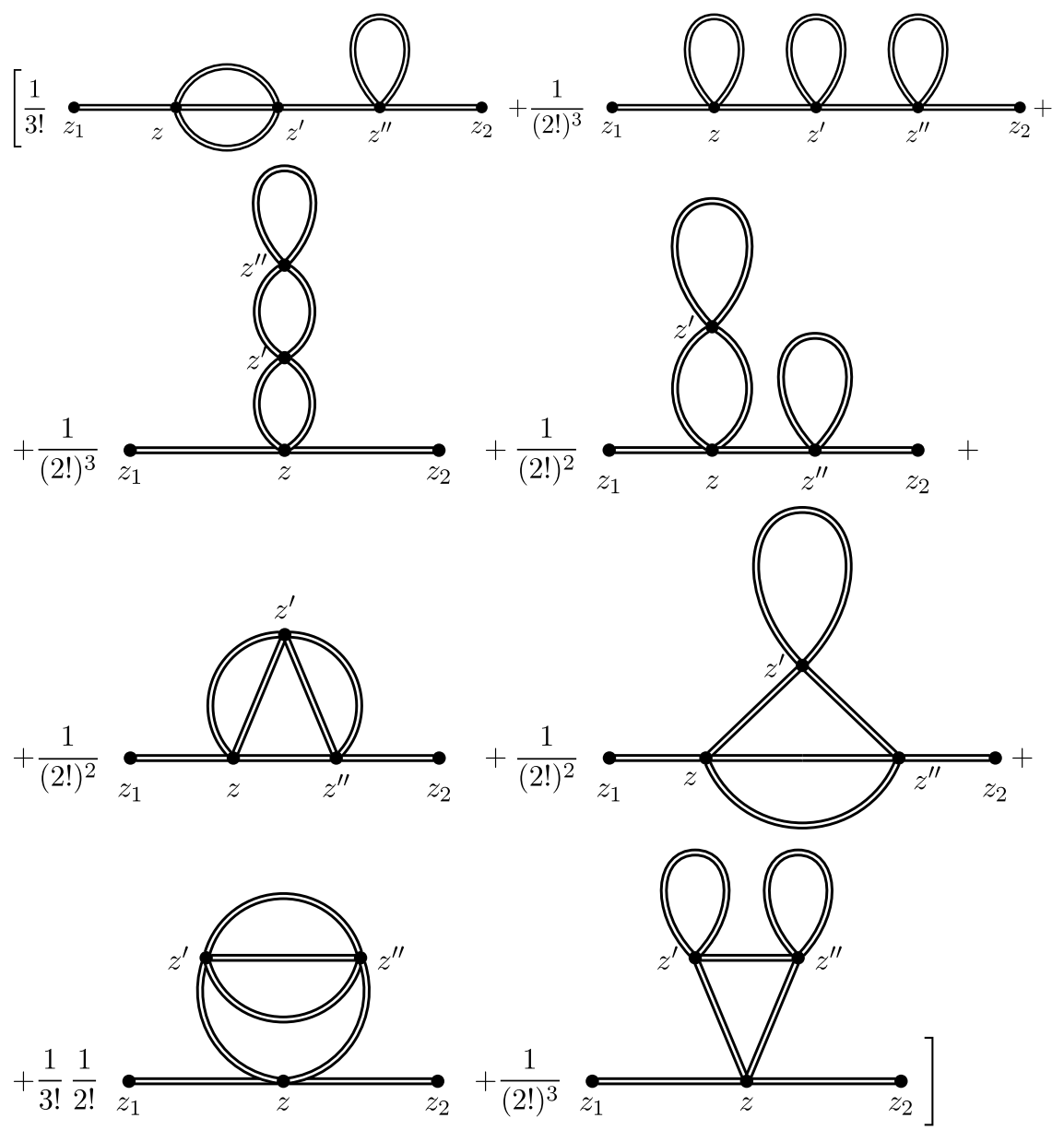

Also, by considering the first and fourth diagrams in Eq. (E.1), we note that the diagrams obtained by the interchange $\left(z_{1} \leftrightarrow z_{2}\right)$ gives a contribution. So all together we end up with ten super-diagrams at 
the third order. (In order to get the $\langle\phi \phi\rangle$ components of these graphs we have to integrate them with the projector $\bar{\theta}_{1} \theta_{1} \bar{\theta}_{2} \theta_{2}$ like we did in (5.21).)

The calculations are long but straightforward and the result is
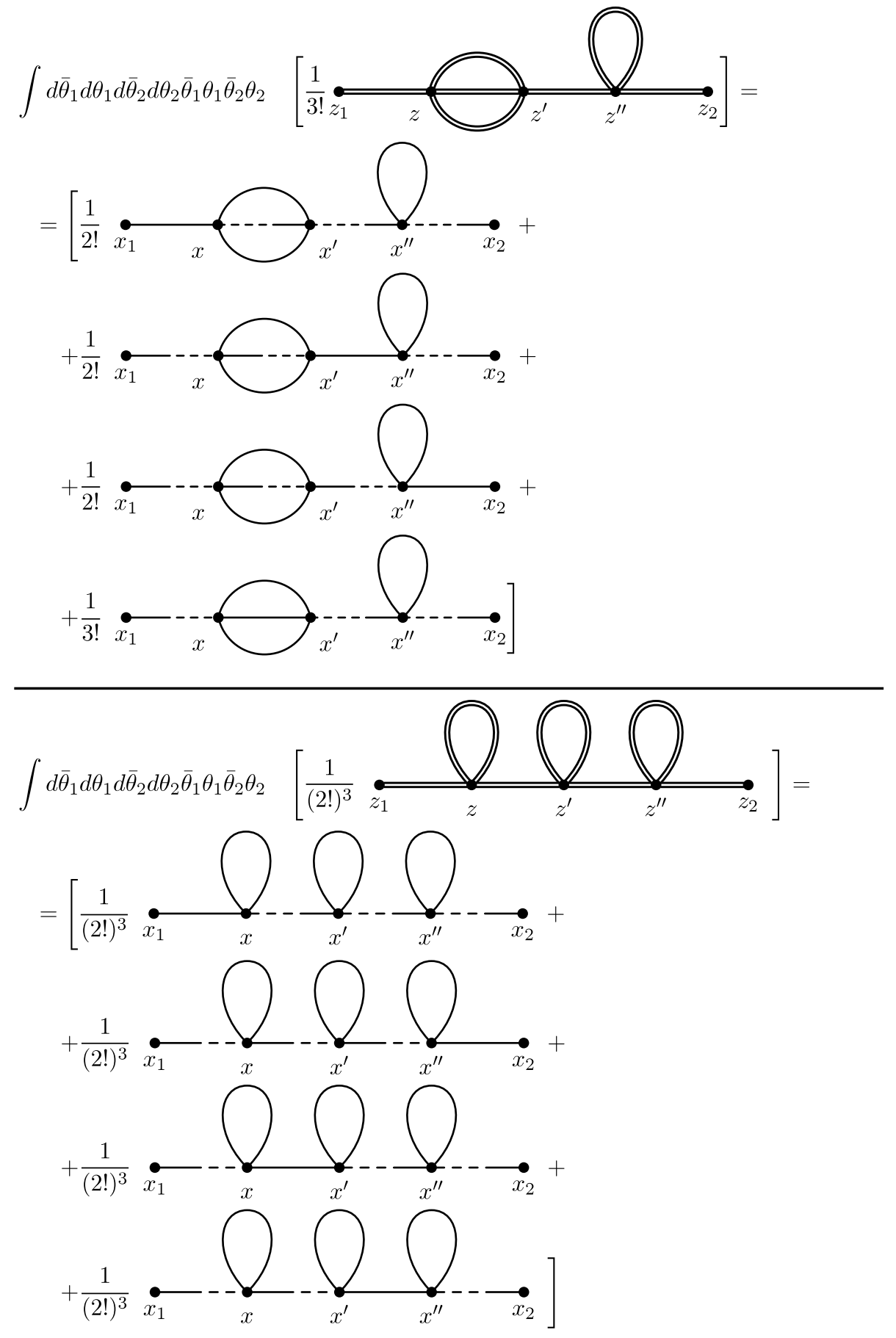


$\frac{8}{8}$


2418

E. Cattaruzza et al. / Annals of Physics 326 (2011) 2377-2430
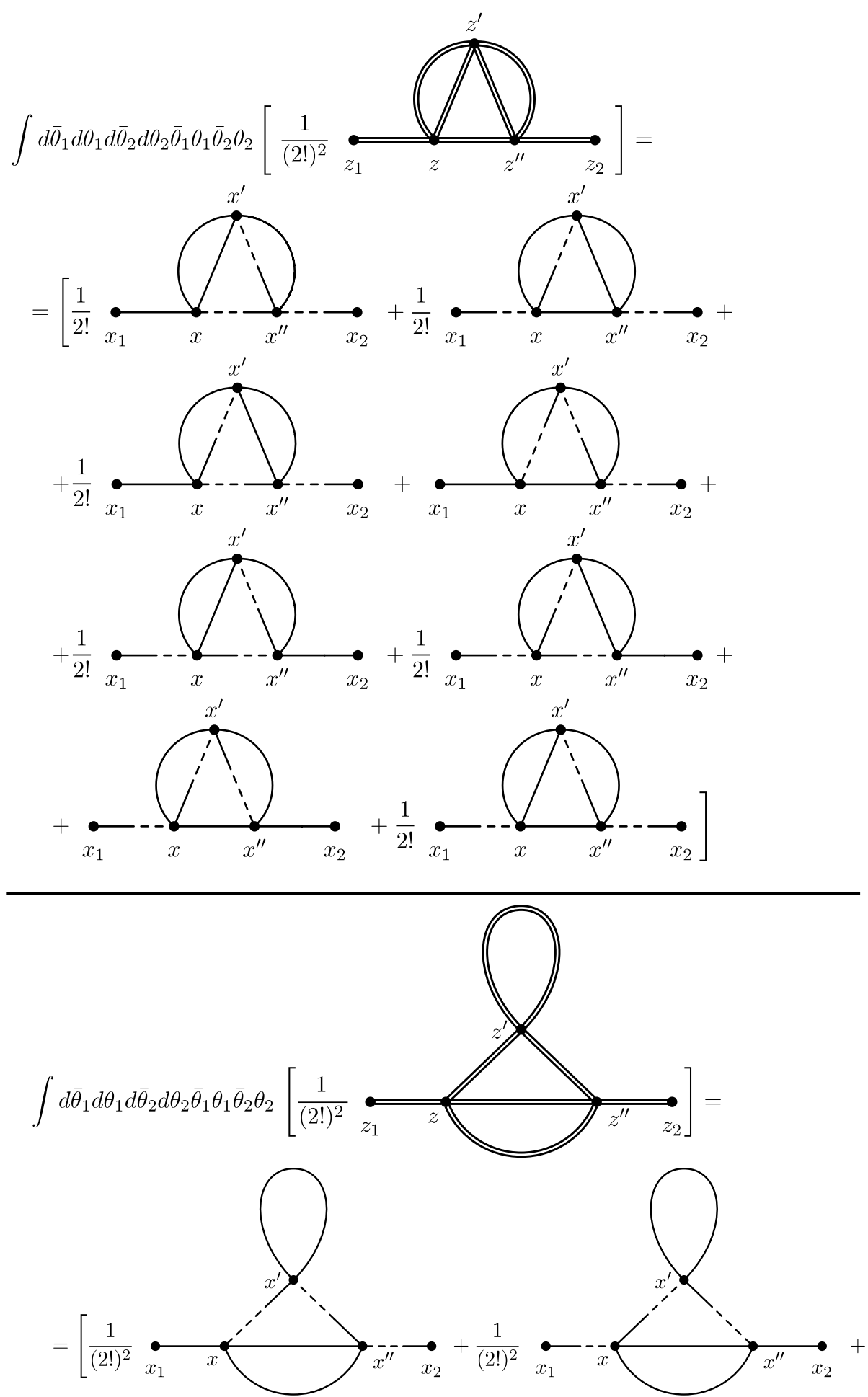

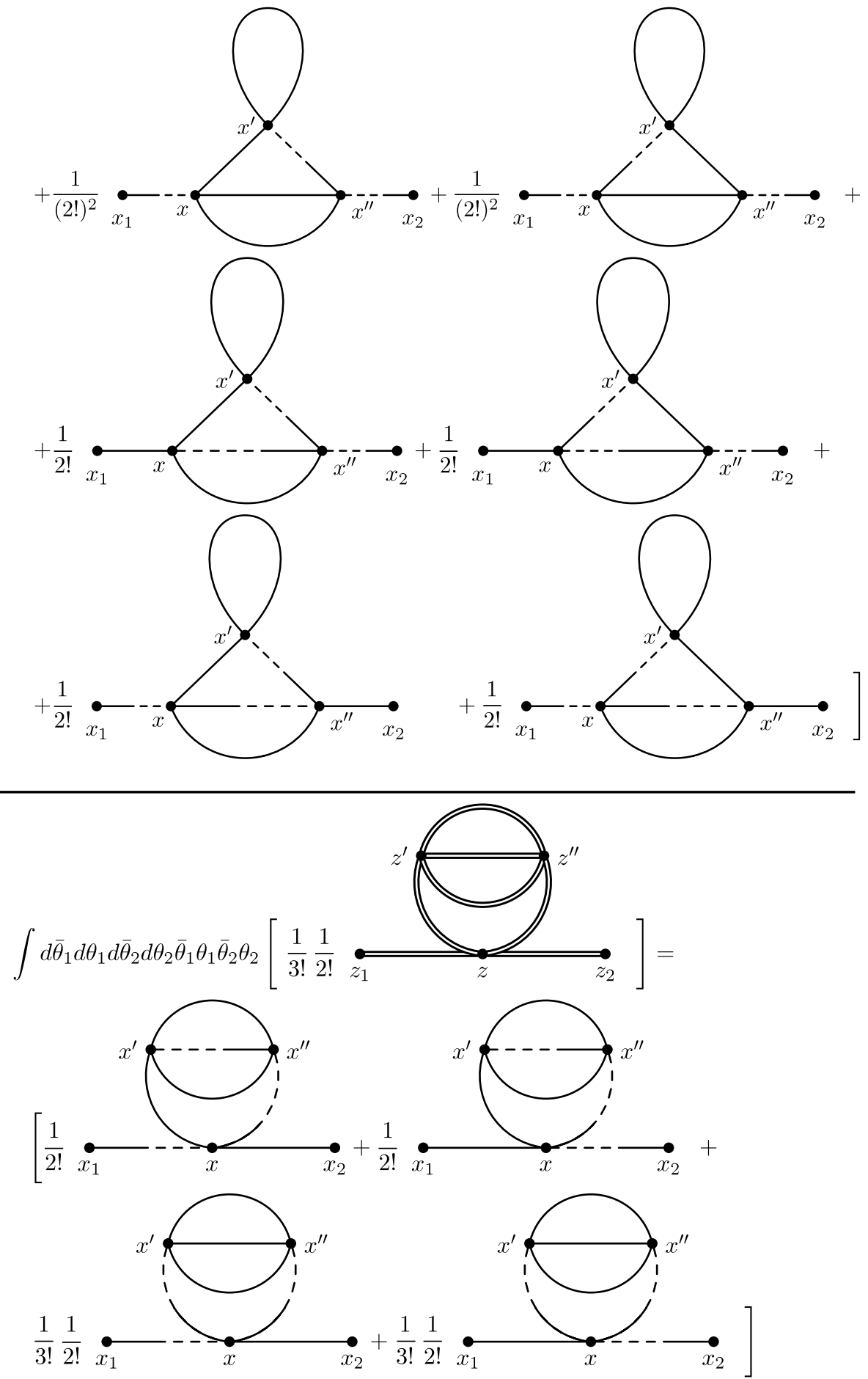


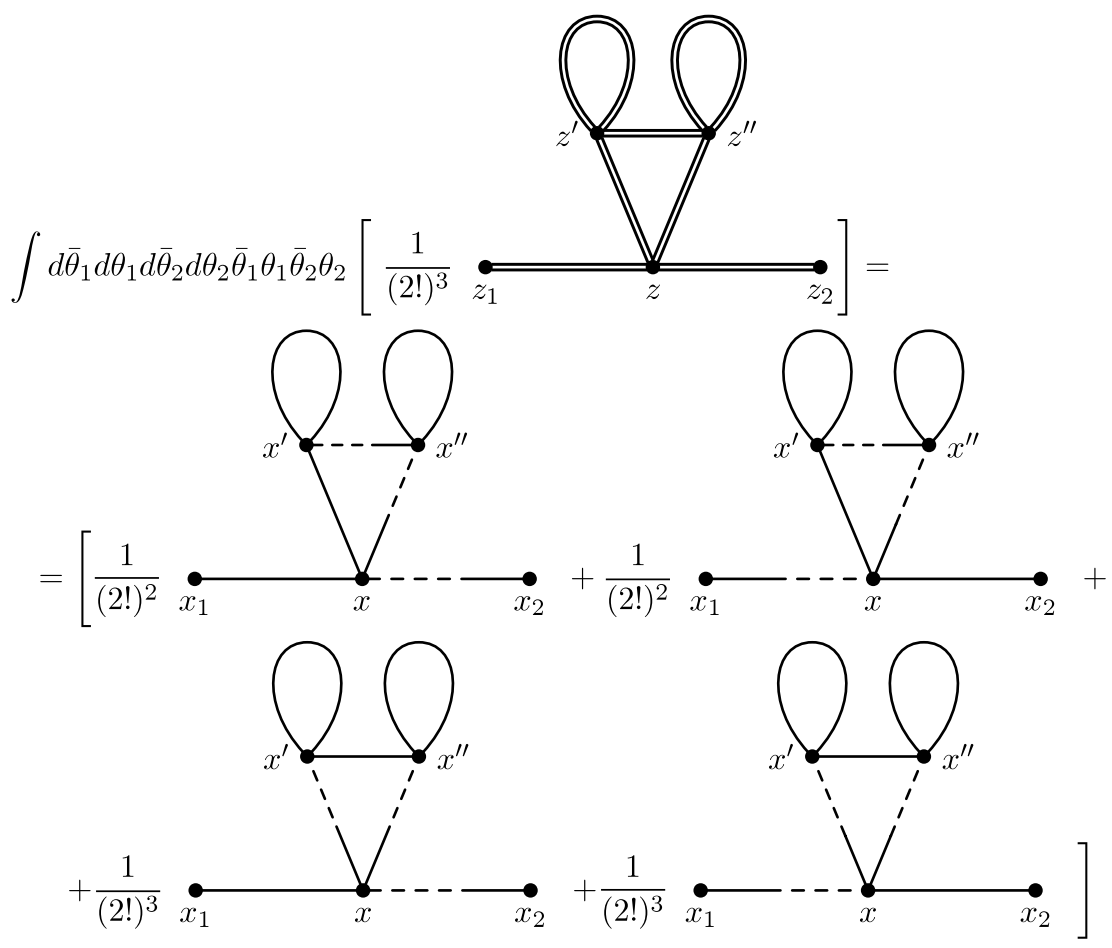

Of course, using the set of super-diagrams (E.1) and different projectors we could get also the $\langle\phi \lambda\rangle$ and $\langle c \bar{c}\rangle$ correlations up to the third order.

\section{Appendix F. Fluctuation-dissipation theorem via Ward identities}

In this appendix we will present a non-perturbative derivation of the fluctuation-dissipation theorem and of other relations that we proved perturbatively in the paper. All these relations are basically Ward identities associated to the various symmetries present in the CPI, i.e., BRS, $\overline{B R S}$, Susy, etc. Most of the calculations presented in this appendix were done long ago by Martin Reuter whom we wish to warmly thank for his generosity.

In this appendix we will not work in the proper KvN formalism with an Hilbert space, etc, but in the Liouville one where a probability density $\tilde{\rho}(\varphi)$ is used.

Let us suppose we give the initial probability density $\tilde{\rho}(-\infty)$ at time $t=-\infty$ and that we want to evaluate the average of a quantity $\mathcal{O}$. The path-integral expression for this average is:

$$
\langle\mathcal{O}\rangle_{\tilde{\rho}(-\infty)}=\int \mathscr{D} \varphi \mathscr{D} \lambda \mathscr{D} \boldsymbol{C} \mathscr{D} \overline{\mathcal{O}} \tilde{\rho}(\varphi(-\infty), c(-\infty)) \exp \left(i \int_{-\infty}^{\infty} d t \tilde{\mathcal{L}}\right) .
$$

Let us ask for what symmetry transformation (whose infinitesimal transformation we indicate with $\delta$ ) and $\tilde{\rho}$ we have

$$
\langle\delta \mathcal{O}\rangle_{\tilde{\rho}(-\infty)}=0 .
$$

Let us assume that the integration measure $\mathscr{D} \varphi \mathscr{D} \lambda \mathscr{D C} \mathscr{D} \bar{C}$ is invariant under the transformation $\delta$ and that the action $\tilde{s}=\int \tilde{\mathcal{L}} d t$ changes only by a surface term

$$
\delta \tilde{s}=\epsilon \sigma(+\infty)-\epsilon \sigma(-\infty),
$$

where $\epsilon$ is defined by $\varphi_{\epsilon}=\varphi+\delta \epsilon$. 
Let us change the variables of integration in (F.1) going over to those obtained via a symmetry transformation. We obtain:

$$
\begin{aligned}
\langle\mathcal{O}\rangle_{\tilde{\rho}(-\infty)} & =\int \mathscr{D} \varphi \ldots(\mathcal{O}+\delta \mathcal{O})[\tilde{\rho}(-\infty)+\delta \tilde{\rho}(-\infty)] \exp (i \tilde{\delta}+i \epsilon \sigma(+\infty)-i \epsilon \sigma(-\infty)) \\
& =\int \mathscr{D} \varphi \ldots\{\mathcal{O} \tilde{\rho}+\mathcal{O} \delta \tilde{\rho}+\tilde{\rho} \delta \mathcal{O}+\mathcal{O} \tilde{\rho}[i \epsilon \sigma(+\infty)-i \epsilon \sigma(-\infty)]\} e^{i \tilde{\delta}}
\end{aligned}
$$

The first term inside the integral fives $\langle\mathcal{O}\rangle_{\tilde{\rho}(-\infty)}$ which is equal to the LHS because we did only a change in integration variables and so these two terms cancel. In this manner we get for the third term in the integral the following expression:

$$
\langle\delta \mathcal{O}\rangle_{\tilde{\rho}(-\infty)}=-\int \mathscr{D} \varphi \ldots \mathcal{O}\{\delta \tilde{\rho}(-\infty)+\tilde{\rho}(-\infty) i \epsilon[\sigma(+\infty)-\sigma(-\infty)]\} e^{i \tilde{\gamma}} .
$$

For $\delta$ let us use the transformation generated by the supersymmetric charges [11]

$$
\begin{aligned}
& Q_{H}=c^{a}\left(i \lambda_{a}-\beta \partial_{a} H\right) \\
& \bar{Q}_{H}=\bar{c}_{a} \omega^{a b}\left(i \lambda_{b}+\beta \partial_{b} H\right)
\end{aligned}
$$

where $\beta$ is a real parameter which, when is set to zero, gives the BRS, $\overline{\mathrm{BRS}}$ [11] charges. Let us now proceed to find the surface terms associated to this symmetry transformations:

$$
\begin{aligned}
& \delta_{Q_{H}} \tilde{\mathcal{L}}=-i \beta \frac{d}{d t} \epsilon N \\
& \delta_{\bar{Q}_{H}} \tilde{\mathcal{L}}=-i \beta \frac{d}{d t} \bar{\epsilon} \bar{Q}
\end{aligned}
$$

where $N \equiv c^{a} \partial_{a} H$ and $\bar{Q} \equiv \bar{c}_{a} \omega^{a b} i \lambda_{b}$. Let us now calculate the first term inside the brackets on the r.h.s of Eq. (F.5) for the transformation generated by $Q_{H}$

$$
\delta \tilde{\rho}(-\infty)=\left(\delta \varphi^{a} \partial_{a} \tilde{\rho}+\delta c^{a} \frac{\partial}{\partial c^{a}} \tilde{\rho}\right)(t=-\infty)=\epsilon c^{a}(-\infty) \partial_{a} \tilde{\rho}(\varphi(-\infty), c(-\infty)) .
$$

The surface term inside the brackets of Eq. (F.5) gives

$$
i \epsilon \sigma(+\infty)-i \epsilon \sigma(-\infty)=\beta \epsilon\left(c^{a} \partial_{a} H\right)(+\infty)-\beta \epsilon\left(c^{a} \partial_{a} H\right)(-\infty) .
$$

Inserting all this in (F.5) we get:

$$
\begin{aligned}
\left\langle\delta_{Q_{H}} \mathcal{O}\right\rangle_{\tilde{\rho}}= & -\int \mathscr{D} \varphi \ldots \mathcal{O} \epsilon\left[c^{a}(-\infty) \frac{\partial}{\partial \varphi^{a}(-\infty)}-\beta c^{a}(-\infty) \partial_{a} H(\varphi(-\infty))\right] \\
& \times \tilde{\rho}(\varphi(-\infty), c(-\infty)) e^{i \tilde{\delta}}-\int \mathscr{D} \varphi \ldots \mathcal{O} \beta \epsilon c^{a}(+\infty) \partial_{a} H(\varphi(+\infty)) \tilde{\rho}(-\infty) e^{i \tilde{s}} \\
= & -\int \mathscr{D} \varphi \mathcal{O} \epsilon \hat{Q}_{H} \tilde{\rho} e^{i \tilde{\delta}}-\beta \int \mathscr{D} \varphi \ldots \epsilon c^{a} \partial_{a} H \mathcal{O} \tilde{\rho} e^{i \tilde{\delta}}
\end{aligned}
$$

For $\beta=0$ the second term vanish and the first is zero only if

$$
\hat{Q} \tilde{\rho}=0
$$

where $\hat{Q}=\left.\hat{Q}_{H}\right|_{\beta=0}=0$. The solution of (F.13) is

$$
\tilde{\rho}=\rho(\varphi) \delta(c) \text {. }
$$

Let us do the analog for the transformation generated by $\bar{Q}_{H}$. Calculating the various pieces we get

$$
\left\langle\delta_{\bar{Q}_{H}} \mathcal{O}\right\rangle_{\tilde{\rho}} \equiv-\int \mathscr{D} \varphi \ldots \mathcal{O}\left(\epsilon \bar{Q}_{H} \tilde{\rho}\right)(-\infty) e^{i \tilde{\S}}
$$


This implies that $\left\langle\delta_{\bar{Q}_{H}} \mathcal{O}\right\rangle_{\tilde{\rho}}=0$ if

$$
\bar{Q}_{H} \tilde{\rho}=0 .
$$

The solution of this equation was calculated in the second of Ref. [11] and is the canonical distribution

$$
\tilde{\rho}(\varphi, c)=e^{-\beta H} \delta(c) .
$$

So the averages taken with this $\tilde{\rho}$ are exactly the thermal averages we have calculated all along in the core of the paper.

Let us now choose as operator $\mathcal{O}$ the following one

$$
\mathcal{O} \equiv \varphi^{a}(t) c^{b}(0), \quad t>0 .
$$

Doing the $\bar{Q}_{H}$ transformation of $\mathcal{O}$ we get:

$$
\delta_{\bar{Q}_{H}} \mathcal{O}=-\bar{\epsilon} \omega^{a d} \bar{c}_{d}(t) c^{b}(0)+\varphi^{a}(t) \epsilon \omega^{b d}\left[i \lambda_{d}(0)+\beta \partial_{d} H(0)\right] .
$$

It was proved in $[28,29,21]$ that

$$
\left\langle\bar{c}_{d}(t) c^{b}(0)\right\rangle=0
$$

if $t>0$, where the average is taken with the canonical average (F.17). So we get from (F.19)

$$
\left\langle\delta_{\bar{Q}_{H}} \mathcal{O}\right\rangle=0=\bar{\epsilon} \omega^{b d}\left\langle\varphi^{a}(t)\left[i \lambda_{d}(0)+\beta \partial_{d} H(0)\right]\right\rangle
$$

which implies:

$$
\left\langle\varphi^{a}(t) i \lambda_{d}(0)\right\rangle=-\beta\left\langle\varphi^{a}(t) \partial_{d} H(0)\right\rangle .
$$

From the equation of motion we have

$$
\partial_{d} H(0)=\omega_{d e} \dot{\varphi}^{e}(0) .
$$

So (F.22) will become

$$
\left\langle\varphi^{a}(t) i \lambda_{d}(0)\right\rangle=-\beta \omega_{d e}\left\langle\varphi^{a}(t) \dot{\varphi}^{e}(0)\right\rangle .
$$

Now, in general, for time-independent Hamiltonians we have, considering two observables $A(t)$ and $B(t)$,

$$
\left\langle A\left(t_{1}\right) B\left(t_{2}\right)\right\rangle=F\left(t_{1}-t_{2}\right)
$$

so

$$
\frac{\partial}{\partial t_{1}}\left\langle A\left(t_{1}\right) B\left(t_{2}\right)\right\rangle=-\frac{\partial}{\partial t_{2}}\left\langle A\left(t_{1}\right) B\left(t_{2}\right)\right\rangle
$$

or

$$
\left\langle\dot{A}\left(t_{1}\right) B\left(t_{2}\right)\right\rangle=-\left\langle A\left(t_{1}\right) \dot{B}\left(t_{2}\right)\right\rangle .
$$

Applying this last relation to (F.24) we get:

$$
\left\langle\varphi^{a}(t) i \lambda_{d}(0)\right\rangle=\beta \omega_{d e} \frac{\partial}{\partial t}\left\langle\varphi^{a}(t) \varphi^{e}(0)\right\rangle .
$$

and this is the fluctuation-dissipation theorem.

With respect to the easier proof presented in [15] our proof has the virtue that it can be seen as a Ward identity of Susy and that the canonical distribution is not put by hand, but it is derived [11] as a supersymmetric invariant state.

Let us now prove few more identities. Let us choose $\tilde{\rho}(\varphi, c)=\delta^{2 n}(c(-\infty))$. This distribution is BRS invariant [11] so:

$$
\left\langle\delta_{\mathrm{BRS}} \mathcal{O}\right\rangle=\int \mathscr{D} \varphi \ldots\left(\delta_{\mathrm{BRS}} \mathcal{O}\right) \delta^{2 n} c(-\infty) e^{i \tilde{\mathcal{S}}}=0
$$


where the BRS transformation [11] is

$$
\left\{\begin{array}{l}
\delta \varphi^{a}=\epsilon c^{a} \\
\delta c^{a}=0 \\
\delta \bar{c}_{a}=i \epsilon \lambda_{a} \\
\delta \lambda_{a}=0
\end{array}\right.
$$

Let us take $\hat{\mathcal{O}}=\varphi^{a}(t) \bar{c}_{b}(0)$, then

$$
\delta \hat{\mathcal{O}}=-i \epsilon\left[i c^{a}(t) \bar{c}_{b}(0)-\varphi^{a}(t) \lambda_{b}(0)\right] .
$$

So $\langle\delta \hat{\mathcal{O}}\rangle=0$ implies

$$
\left\langle\varphi^{a}(t) \lambda_{b}(0)\right\rangle=i\left\langle c^{a}(t) \bar{c}_{b}(0)\right\rangle .
$$

This relation tell us that the dash-full propagator is equal to the Fermion one and to all orders in perturbation theory. This confirms the statement that we made all along in our paper that this identity is related to the Susy invariance (better say to the BRS invariance which is the Susy with $\beta=0$ ).

Let us now choose as operator

$$
\hat{\mathcal{O}}=\lambda_{a}(t) \bar{c}_{b}(0)
$$

then

$$
\delta \hat{\mathcal{O}}=i \in \lambda_{a}(t) \lambda_{a}(0),
$$

and from $\langle\delta \hat{\mathcal{O}}\rangle=0$ we obtain:

$$
\left\langle\lambda_{a}(t) \lambda_{a}(0)\right\rangle=0 \text {. }
$$

This is "almost" similar to the one we proved in the paper perturbatively to all orders, because there we proved it with the weight $\rho=e^{-\beta H} \delta^{n}(c)$, while here we proved it only for $\beta=0$, but we think the proof given here should go through also for $\beta \neq 0$.

Let us now choose as operator

$$
\hat{\mathcal{O}}=c^{a}(t) \varphi^{b}(0),
$$

then

$$
\delta \hat{\mathcal{O}}=-\epsilon c^{a}(t) c^{b}(0),
$$

so we get

$$
\left\langle c^{a}(t) c^{b}(0)\right\rangle=0 .
$$

This relation for $a=b=1$ was proved to all orders of perturbation theory in the paper and for the canonical distribution. Here we have given a non-perturbative proof.

We like to conclude this appendix by saying that both the simplification in the diagrammatics that we got via the introduction of super-diagrams and the various identities we found that will further simplify the diagrammatics, have their roots in the various symmetries of the CPI.

\section{Appendix G. Perturbative check of the fluctuation-dissipation theorem}

In this appendix we will prove the relations (6.11) and (6.12).

Let us remember that

$$
\Delta_{\beta}\left(x_{1}-x_{2}\right)=\int \frac{d^{4} p}{(2 \pi)^{4}} \frac{2 \pi}{\beta\left|p^{0}\right|} \delta\left(p^{2}-m^{2}\right) e^{-i p \cdot x}
$$

where $p \cdot x=p^{0} t-\vec{p} \cdot \vec{x}$ and $p^{2}=\left(p^{0}\right)^{2}-\vec{p}^{2}$. 
Let us take the derivative with respect to $t_{1}$, like on the 1.h.s of Eq. (6.11)

$$
\frac{\partial}{\partial t_{1}} \Delta_{\beta}\left(x_{1}-x_{2}\right)=-i \int \frac{d^{4} p}{(2 \pi)^{4}} \frac{2 \pi}{\beta} \frac{p^{0}}{\left|p^{0}\right|} \delta\left(p^{2}-m^{2}\right) e^{-i p \cdot x} .
$$

Let us remember that:

$$
\frac{p^{0}}{\left|p^{0}\right|}=\theta\left(p^{0}\right)-\theta\left(-p^{0}\right) \equiv \Theta\left(p^{0}\right),
$$

where we have defined the new symbol $\Theta\left(p^{0}\right)$. Let us also notice that:

$$
\delta\left(\left(p^{0}\right)^{2}-\vec{p}^{2}-m^{2}\right)=\frac{1}{2 E_{\vec{p}}}\left[\delta\left(p^{0}-E_{\vec{p}}\right)-\delta\left(p^{0}+E_{\vec{p}}\right)\right],
$$

and so we get:

$$
\Theta\left(p^{0}\right) \delta\left(\left(p^{0}\right)^{2}-\vec{p}^{2}-m^{2}\right)=\frac{1}{2 E_{\vec{p}}}\left[\delta\left(p^{0}-E_{\vec{p}}\right)-\delta\left(p^{0}+E_{\vec{p}}\right)\right] .
$$

We can now rewrite (G.2) as

$$
\begin{aligned}
\frac{\partial}{\partial t_{1}} \Delta_{\beta}\left(x_{1}-x_{2}\right)= & -i \frac{1}{\beta} \int \frac{d^{3} \vec{p}}{(2 \pi)^{3}} \frac{1}{2 E_{\vec{p}}} e^{-i E_{\vec{p}}\left(t_{2}-t_{1}\right)} e^{i \vec{p} \cdot\left(\vec{x}_{1}-\vec{x}_{2}\right)} \\
& +i \frac{1}{\beta} \int \frac{d^{3} \vec{p}}{(2 \pi)^{3}} \frac{1}{2 E_{\vec{p}}} e^{i E_{\vec{p}}\left(t_{1}-t_{2}\right)} e^{i \vec{p} \cdot\left(\vec{x}_{1}-\vec{x}_{2}\right)} .
\end{aligned}
$$

Let us now turn to the r.h.s. of Eq. (6.11) and let us remember that $G_{R}$ solves the equation:

$$
\left(\square+m^{2}\right) G_{R}=-\delta(x)
$$

with $G_{R}(x)=0$ for $t<0$. Let us then choose the contour $C_{R}$ of integration along the real axis in the complex $p$ plane in such a manner that the two poles are moved below the real axis.

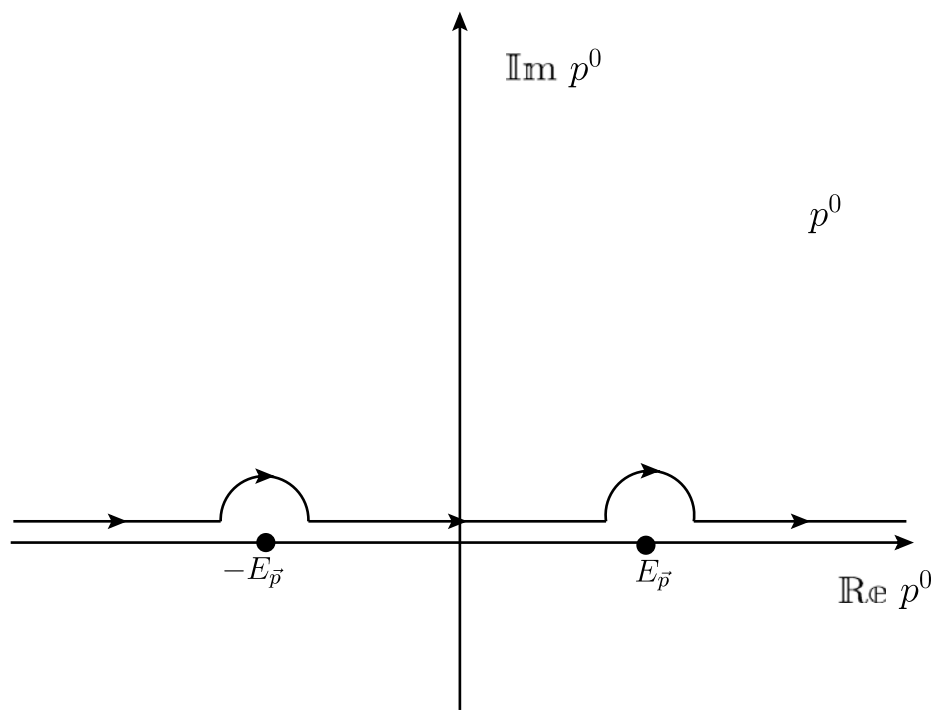

For $t>0$ we close the contour in the lower half plane:

$$
G_{R}(x)=\int_{-\infty}^{\infty} \frac{d^{4} p}{(2 \pi)^{4}} \frac{e^{-i p x}}{\left(p^{0}+i \epsilon\right)^{2}-E_{\vec{p}}^{2}}=\int_{C_{R}} \frac{d^{4} p}{(2 \pi)^{4}} \frac{e^{-i p x}}{\left(p^{0}+i \epsilon\right)^{2}-E_{\vec{p}}^{2}} .
$$


So by the residue theorem we get:

$$
G_{R}(x)=-i \int_{-\infty}^{\infty} \frac{d^{3} \vec{p}}{(2 \pi)^{3}} \frac{1}{2 E_{\vec{p}}} e^{-i E_{\vec{p}} t-\vec{p} \cdot \vec{x}}+i \int_{-\infty}^{\infty} \frac{d^{3} \vec{p}}{(2 \pi)^{3}} \frac{1}{2 E_{\vec{p}}} e^{i E_{\vec{p}} t-\vec{p} \cdot \vec{x}}
$$

and comparing with Eq. (G.6) we obtain:

$$
G_{R}(x)=G_{R}\left(x_{1}-x_{2}\right)=\beta \frac{\partial}{\partial t_{1}} \Delta_{\beta}\left(x_{1}-x_{2}\right) .
$$

For $t<0$ we get that $G_{R}(x)$ is zero, so:

$$
G_{R}(x)=\beta \theta(t) \frac{\partial}{\partial t} \Delta_{\beta}(x) .
$$

In this relation let us now take $x \rightarrow-x$ and we get:

$$
G_{R}(-x)=\beta \theta(-t) \frac{\partial}{\partial(-t)} \Delta_{\beta}(-x)=\beta \theta(t) \frac{\partial}{\partial(-t)} \Delta_{\beta}(x)=-\beta \theta(t) \frac{\partial}{\partial t} \Delta_{\beta}(x) .
$$

So for $t<0$ we have:

$$
\frac{\partial}{\partial t} \Delta_{\beta}(x)=-\frac{1}{\beta} G_{R}(-x) .
$$

Combining (G.11) with (G.13) we can write:

$$
\frac{\partial}{\partial t} \Delta_{\beta}(x)=\frac{1}{\beta} G_{R}(x)-\frac{1}{\beta} G_{R}(-x)
$$

which is exactly the (6.11) or the FDT theorem at the zero order that is what we wanted to prove.

We will now move on to prove Eq. (6.12). Let us first note that, from (G.14) we obtain:

$$
G_{R}\left(x_{1}-x\right)=\beta \theta\left(x_{1}-x\right) \frac{\partial}{\partial t_{1}} \Delta_{\beta}\left(x_{1}-x\right) .
$$

The first term on the 1.h.s. of (6.12) has the following analytical expression:

$$
\begin{aligned}
& \frac{1}{2} \frac{\partial}{\partial t_{1}} \\
& \quad=\frac{1}{2} \int d^{4} x \frac{1}{\beta}\left[G_{R}^{(B)}\left(x_{1}-x\right)-G_{R}^{(B)}\left(x-x_{1}\right)\right] \Delta_{\beta}(x-x) G_{R}^{(B)}\left(x_{2}-x\right)
\end{aligned}
$$

where we have made use of the relation (G.14).

The second term on the l.h.s. of (6.12) has the following analytical expression:

$$
\frac{1}{2} \frac{\partial}{\partial t_{1}} \underset{x_{1}}{\bullet}=\frac{1}{2} \int d^{4} x \frac{\partial}{\partial t_{1}} G_{R}^{(B)}\left(x_{1}-x\right) \Delta_{\beta}(x-x) \Delta_{\beta}\left(x-x_{2}\right) .
$$

Let us now take the time derivative of (G.15):

$$
\frac{\partial}{\partial t_{1}} G_{R}^{(B)}\left(x_{1}-x\right)=\beta \delta\left(t_{1}-t\right) \frac{\partial}{\partial t_{1}} \Delta_{\beta}\left(x_{1}-x\right)+\beta \theta\left(t_{1}-t\right) \frac{\partial^{2}}{\partial t_{1}^{2}} \Delta_{\beta}\left(x_{1}-x\right) .
$$

Moreover, it is easy to prove that

$$
\delta\left(t_{1}-t\right) \frac{\partial}{\partial t_{1}} \Delta_{\beta}\left(x_{1}-x\right)=0,
$$


and therefore, (G.18) becomes

$$
\frac{\partial}{\partial t_{1}} G_{R}^{(B)}\left(x_{1}-x\right)=\beta \theta\left(t_{1}-t\right) \frac{\partial^{2}}{\partial t_{1}^{2}} \Delta_{\beta}\left(x_{1}-x\right) .
$$

As a consequence the (G.17) is turned into:

$$
\begin{aligned}
& \frac{1}{2} \frac{\partial}{\partial t_{1}} x_{1} \\
& \quad=\frac{1}{2} \beta \int d^{4} x \theta\left(t_{1}-t\right) \frac{\partial^{2}}{\partial t_{1}^{2}} \Delta_{\beta}\left(x_{1}-x\right) \Delta_{\beta}(x-x) \Delta_{\beta}\left(x-x_{2}\right) .
\end{aligned}
$$

Performing now an integration by parts, and disregarding surface terms, Eq. (G.21) can be rewritten as

$$
\begin{aligned}
& \frac{1}{2} \frac{\partial}{\partial t_{1}} \underset{x_{1}}{\bullet} x_{2}=-\frac{1}{2} \beta \int d^{4} x \delta\left(t_{1}-t\right) \Delta_{\beta t_{1}}\left(x_{1}-x\right) \Delta_{\beta}(x-x) \Delta_{\beta}\left(x_{2}-x\right) \\
& -\frac{1}{2} \beta \int d^{4} x \theta\left(t_{1}-t\right) \Delta_{\beta}\left(x_{1}-x\right) \Delta_{\beta}(x-x) \Delta_{\beta t_{2}}\left(x_{2}-x\right)
\end{aligned}
$$

where $\Delta_{\beta} t_{i}\left(x_{i}-x\right) \equiv \partial \Delta_{\beta}\left(x_{i}-x\right) / \partial t_{i}$. Using (G.19) we get:

$$
\begin{aligned}
& \frac{1}{2} \frac{\partial}{\partial t_{1}} \underset{x_{1}}{\longrightarrow}=-\frac{1}{2} \beta \int d^{4} x \theta\left(t_{1}-t\right) \Delta_{\beta} t_{1}\left(x_{1}-x\right) \Delta_{\beta}(x-x) \Delta_{\beta} t_{2}\left(x_{2}-x\right) .
\end{aligned}
$$

If on the r.h.s. of the equation above we use (G.14), we obtain:

$$
\begin{aligned}
& \frac{1}{2} \frac{\partial}{\partial t_{1}} \underset{x_{1}}{\bullet}=-\frac{1}{2} \frac{1}{\beta} \int d^{4} x G_{R}^{(B)}\left(x_{1}-x\right) \Delta_{\beta}(x-x)\left[G_{R}^{(B)}\left(x_{2}-x\right)-G_{R}^{(B)}\left(x-x_{2}\right)\right] .
\end{aligned}
$$

Combining (G.16) with (G.24) we get:

$$
\begin{aligned}
\frac{1}{2} & \frac{\partial}{\partial t_{1}} \\
= & \frac{1}{2} \frac{1}{\beta} \int d^{4} x G_{R}^{(B)}\left(x_{1}-x\right) \Delta_{\beta}(x-x) G_{R}^{(B)}\left(x-x_{2}\right) \\
& -\frac{1}{2} \frac{1}{\beta} \int d^{4} x G_{R}^{(B)}\left(x-x_{1}\right) \Delta_{\beta}(x-x) G_{R}^{(B)}\left(x_{2}-x\right) \\
= & {\left[\frac{i}{2 \beta} \stackrel{\bullet}{x_{1}}\right.}
\end{aligned}
$$

This is exactly the relation (6.12) which is the FDT of the first order in perturbation theory. 
Long but tedious calculations allows us to derive the FDT also at the second order in perturbation theory which is diagrammatically represented by the three formulas (6.13)-(6.15).

For the first order contribution to the FDT an alternative and simpler derivation than the one presented before can be given either using the momentum representation or the super-field formalism. We will present them below:

Momentum space derivation: Eq. (6.11), which is the FDT at zero order, is:

$$
\frac{\partial}{\partial t_{1}} \Delta_{\beta}\left(x_{1}-x_{2}\right)=\frac{i}{\beta}\left[-i G_{R}\left(x_{1}-x_{2}\right)+i G_{R}\left(x_{2}-x_{1}\right)\right] .
$$

Going into momentum space we get:

$$
\begin{aligned}
& \frac{\partial}{\partial t_{1}} \int \frac{d^{4} p}{(2 \pi)^{4}} e^{-i p \cdot\left(x_{1}-x_{2}\right)} \Delta_{\beta}(p) \\
& \quad=\frac{i}{\beta}\left\{\int \frac{d^{4} p}{(2 \pi)^{4}} e^{-i p \cdot\left(x_{1}-x_{2}\right)}\left[-i G_{R}(p)\right]+\int \frac{d^{4} p}{(2 \pi)^{4}} e^{-i p \cdot\left(x_{1}-x_{2}\right)}\left[i G_{R}(-p)\right]\right\},
\end{aligned}
$$

or equivalently:

$$
\begin{aligned}
& \int \frac{d^{4} p}{(2 \pi)^{4}}\left[-i p^{0} e^{-i p \cdot\left(x_{1}-x_{2}\right)}\right] \Delta_{\beta}(p) \\
& =\frac{i}{\beta}\left\{\int \frac{d^{4} p}{(2 \pi)^{4}} e^{-i p \cdot\left(x_{1}-x_{2}\right)}\left[-i G_{R}(p)\right]+\int \frac{d^{4} p}{(2 \pi)^{4}} e^{-i p \cdot\left(x_{1}-x_{2}\right)}\left[i G_{A}(p)\right]\right\},
\end{aligned}
$$

where $G_{A}$ is the advanced propagator. Formally, we shall write the relation above as:

$$
-i p^{0} \Delta_{\beta}(p)=\frac{i}{\beta}\left[-i G_{R}(p)+i G_{A}(p)\right]
$$

which diagrammatically is:

$$
-i p^{0} \stackrel{p}{\bullet} \underset{x_{1}}{\bullet} \quad x_{2}=\frac{i}{\beta} \underset{x_{1}}{\bullet}-\stackrel{p}{\bullet}-\frac{i}{\beta} \underset{x_{1}}{\bullet--} \underset{x_{2}}{\bullet}
$$

The FDT at first order (6.12) is given by:

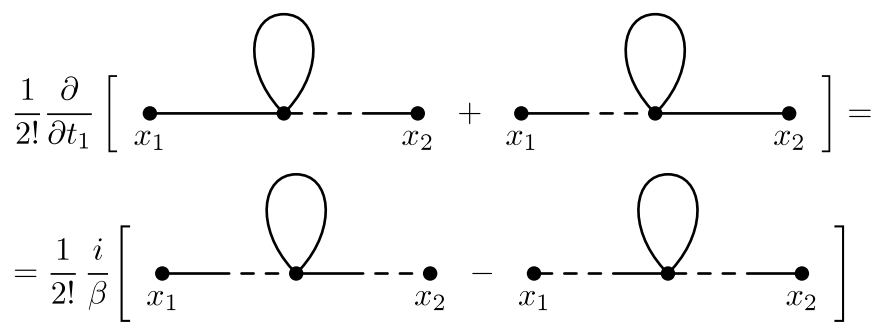

The l.h.s. of (G.31) in momentum space is:

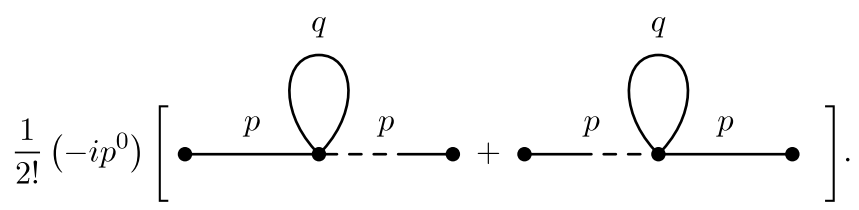

Let us now make use of (G.29) in the following manner: let us replace the first leg of the first diagram in (G.32) using the relation (G.29) and the same for the last leg of the second diagram in (G.32). Therefore, 
we get:

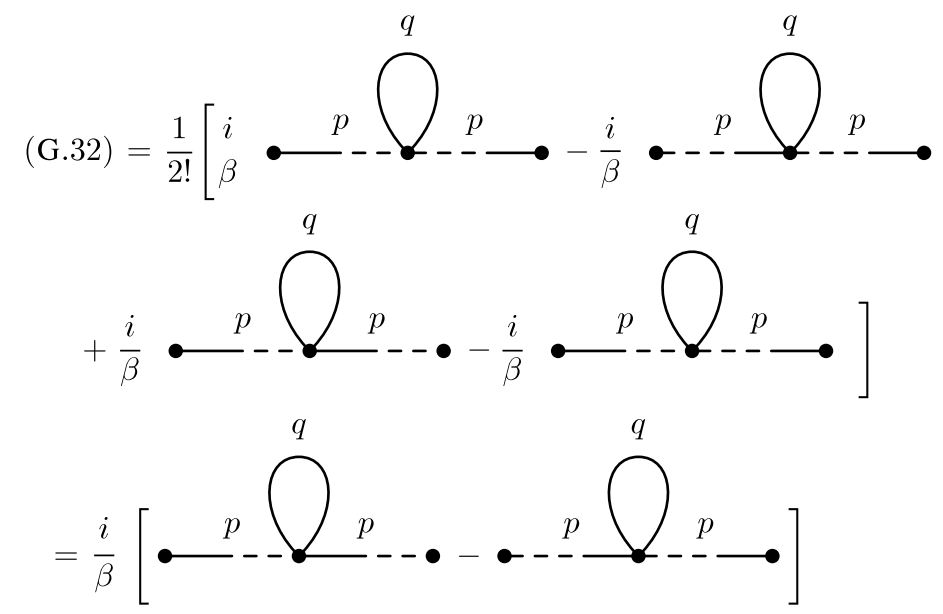

and this is exactly the momentum space representation of (G.31).

One can notice how much simpler is this derivation than the one in configuration space that we presented earlier.

Super-diagram derivation: Let us remember that:

$$
\left\langle\phi\left(x_{1}\right) \phi\left(x_{2}\right)\right\rangle^{(1)}=\frac{1}{2} \int d \nu_{1} d \nu_{2} \underset{z_{1}}{\rightleftharpoons}
$$

where we have used the notation $d v_{i} \equiv d \theta_{i} d \bar{\theta}_{i} \bar{\theta}_{i} \theta_{i}(i=1,2)$ and the upper index (1) on the l.h.s. stays for first order in perturbation theory.

It is then easy to prove that:

$$
\begin{aligned}
& \left\langle\int d \nu_{1} \underset{z_{1}}{\stackrel{\rightleftarrows}{\rightleftarrows}}\right\rangle_{2}=\underset{\beta}{\stackrel{\bullet}{x_{1}} \quad x_{2}}+\underset{x_{1}}{\bullet}-\underset{x_{2}}{\bullet} \bar{\theta}_{2} \theta_{2} \\
& =\int d \nu_{1}\left[\frac{\partial}{\partial \theta_{2}} \frac{\partial}{\partial \bar{\theta}_{2}}\left(\bar{\theta}_{2} \theta_{2} \underset{z_{1}}{z_{2}}\right)+\bar{\theta}_{2} \theta_{2} \frac{\partial}{\partial \theta_{2}} \frac{\partial}{\partial \bar{\theta}_{2}}\left(\underset{z_{1}}{\rightleftharpoons} z_{2}\right)\right] .
\end{aligned}
$$

The first equality above derives from the fact that in $x_{1}$ only the field $\phi$ survives the integration over $d v_{1}$. At the point $z_{2}$ in principle there could be any of the fields $\phi, \lambda, c, \bar{c}$, but only the correlations $\langle\phi \phi\rangle$ and $\langle\phi \lambda\rangle$ survive because the $\langle\phi c\rangle,\langle\phi \bar{c}\rangle$ are zero as we proved before in the paper. The second equality in (G.35) is easy to prove: the first term is exactly equal to the first term of the first equality. In fact, in $z_{2}$ only $\phi$ survives which is the only term which does not carry extra $\theta_{2}$ and in $z_{1}$ only $\phi$ survives because of the integration $d v_{1}$. An analogous reasoning applies to the second term.

Let us now go back to (G.34) and do the time derivative of both, the l.h.s. and r.h.s.

$$
\frac{\partial}{\partial t_{1}}\left\langle\phi\left(x_{1}\right) \phi\left(x_{2}\right)\right\rangle^{(1)}=\frac{1}{2} \int d \nu_{1} d \nu_{2} \underset{z_{1}}{\longrightarrow}
$$

where with the arrow $\Longrightarrow$ we indicate the time derivative. We can formally write the r.h.s. of (G.36) as

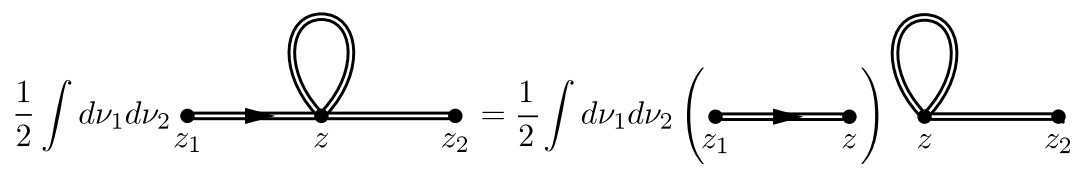


Using the relation (G.35) on the first term of the r.h.s. above, we get:

$$
\begin{aligned}
& \frac{\partial}{\partial t_{1}}\left\langle\phi\left(x_{1}\right) \phi\left(x_{2}\right)\right\rangle^{(1)}=\frac{1}{2} \int d \nu_{1} d \nu_{2}\left[\frac{\partial}{\partial \theta} \frac{\partial}{\partial \bar{\theta}}\left(\bar{\theta} \theta \underset{z}{z_{1} \longrightarrow}\right)\right. \\
& \left.+\bar{\theta} \theta \frac{\partial}{\partial \theta} \frac{\partial}{\partial \bar{\theta}}\left(\underset{z_{1}}{\rightleftharpoons}\right)\right] z_{z}
\end{aligned}
$$

Let us analyze the two terms in the square brackets on the r.h.s. of (G.38).

The first term is equal to

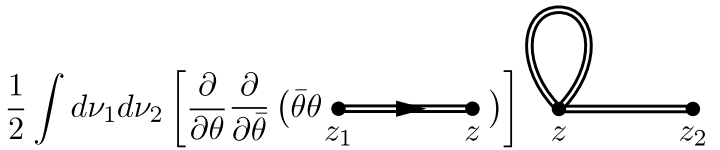

$$
\begin{aligned}
& =\frac{1}{2} \int d \nu_{1} d \nu_{2}\left[\bar{\theta} \theta\left(\underset{z_{1}}{\longrightarrow}\right)\right]\left[\frac{\partial}{\partial \theta} \frac{\partial}{\partial \bar{\theta}}\left(\underset{z}{\longrightarrow} z_{2}\right)\right]
\end{aligned}
$$

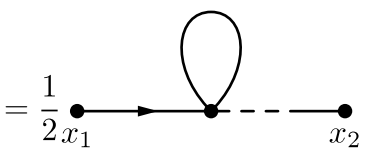

where we have used integration by parts over Grassmann variables to obtain the first equality and we have performed the integration to obtain the second one.

Similarly, the second term in the square brackets on the r.h.s. of (G.38) gives

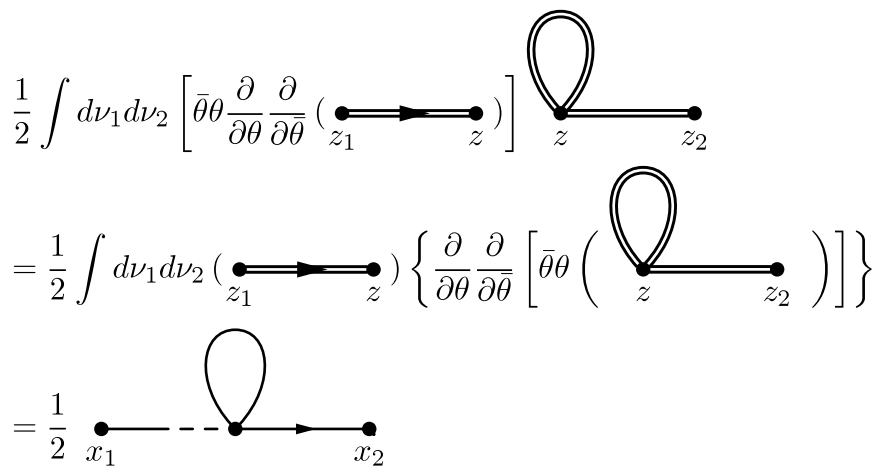

Replacing now in (G.39) and (G.40) the continuous line with the arrow (which stays for the time derivative) with the difference of the dash-full line, like it is indicated in the FDT at zero order (6.11), we get that:

$$
(\mathrm{G} .39)=\left[\frac{i}{2 \beta} \quad: \bullet x_{1}-\frac{i}{x_{2}}-\frac{i}{2 \beta}\right.
$$


Summing up the two relations above we get (6.12) which is the FDT to first order in perturbation theory.

Both, the momentum and the super-field techniques can be used to go to higher order in the FDT. The calculations turn out to be simpler than in standard space, but still rather tedious, so we will avoid to report them here.

This last part of this appendix has been somehow a demonstration of the many things one can prove by "manipulating" the Feynman diagrams without resorting to their analytical expressions. These manipulations are "analogous" to what was done in the original "diagrammar" paper [1] for Quantum Field Theory, so we hope the use we made of the same word "diagrammar" is forgiven.

\section{References}

[1] G.'t Hooft, M. Veltman, Diagrammar CERN 73-9-REPORT.

[2] D. D'enterria, Nuclear Phys. A 782 (2007) 215; M.J. Tannenbaum, Internat. J. Modern Phys. E 17 (2008) 771.

[3] K. Yagi, T. Hatsuda, Y. Miake, Quark-gluon Plasma: From Big Bang to Little Bang, in: Cambridge Monographs on Particle Physics, Nuclear Physics and Cosmology, vol. 23, Cambridge University Press, UK, 2005, p. 446.

[4] S. Galow, et al., Nuclear Phys. B 330 (2010) 834. 0911.0627.

[5] L.V. Gribov, E.R. Levin, M.G. Riskin, Phys. Rep. 100 (1983) 1; J.P. Blaizot, A.H. Mueller, Nuclear Phys. B 239 (1997) 847.

[6] L. McLerran, R. Venugopalan, Phys. Rev. D 49 (1994) 3352;

L. McLerran, R. Venugopalan, Phys. Rev. D 49 (1994) 2233.

[7] G. Aarts, J. Smit, Phys. Lett. B 393 (1997); Nuclear Phys. B 511 (1998) 478; G. Aarts, et al., Phys. Rev. D 61 (2000) 105002; G. Aarts, Phys. Lett. B 518 (2001) 315.

[8] W. Buchmüller, A. Jakovac, Nuclear Phys. B 521 (1998) 219.

[9] A.H. Mueller, D.J. Son, Phys. Lett. B 582 (2004) 279.

[10] S. Jeon, Phys. Rev. C 72 (2005) 014907. hep-ph/0412121.

[11] E. Gozzi, M. Reuter, W.D. Thacker, Phys. Rev D 40 (1989) 3363;

E. Gozzi, M. Reuter, Phys. Lett. B 233 (1989) 383; 240 (1990) 137; A.F. Neto, Rigorous Evaluation of the Jacobi Matrix of Time Evolution (work in progress).

[12] B.O. Koopman, Proc. Natl. Acad. Sci. USA 17 (1931) 315; J. von Neumann, Ann. Math. 33 (1932) 587.

[13] R.P. Feynman, A.R. Hibbs, Quantum Mechanics and Path-Integrals, McGraw-Hill Book Company, New York, 1965.

[14] L.S. Schulman, Techniques and Applications of Path Integrations, Wiley, New York, 1981.

[15] H. Deker, F. Haake, Phys. Rev. A 11 (1975) 2043.

[16] R. Penco, D. Mauro, Eur. J. Phys. 27 (2006) 1241. hep-th/0605061.

[17] P.C. West, Introduction to Supersymmetry and Supergravity, World Scientific, Singapore, 1990.

[18] A.A. Abrikosov Jr., E. Gozzi, D. Mauro, Ann. Phys. 317 (2005) 24. quant-ph/0406028.

[19] R. Abraham, J. Marsden, Foundations of Mechanics, Benjamin, New York, 1978.

[20] H. Nakazato, et al., Nuclear Phys. B 346 (1990) 611.

[21] E. Gozzi, M. Reuter, Chaos Solitons Fractals 4 (7) (1994) 1117. arxiv:nlin/0306042; S. Tanase-Nicola, J. Kurchan, J. Phys. A: Math. Gen. 36 (2003) 10299.

[22] B. Sakita, Quantum Theory of Many-Variable Systems and Fields, World Scientific, Singapore, 1985.

[23] H. Ezawa, J.R. Klauder, Progr. Theoret. Phys. 74 (1989) 904.

[24] M. Sato, Progr. Theoret. Phys. 58 (1977) 1262.

[25] E. Gozzi, C. Pagani, Phys. Rev. Lett. 105 (2010) 150604. arXiv:1006.3029.

[26] E. Gozzi, M. Regini, Phys. Rev. D 62 (2000) 067702. hep-th/9903136; E. Gozzi, D. Mauro, J. Math. Phys. 41 (2000) 1916. hep-th/9907065.

[27] E. Witten, Comm. Math. Phys. 117 (1988) 353.

[28] E. Gozzi, Phys. Rev. D 30 (6) (1984) 1218.

[29] M. Reuter, private communication.

[30] L. Arnold, V. Wihstuz, Lyapunov Exponents, in: Lecture Notes in Mathematics, vol. 1186, Springer, New York, 1996.

[31] E. Gozzi, R. Penco, Ann. of Phys. 326 (2011) 876. arXiv:1008.5135.

[32] H. Wyld, Ann. Phys. 14 (1961) 163.

[33] C. Lenz, L. Garrido, Phys. Rev. A 143 (1976) 1258;

L. Garrido, M. San Miguel, Progr. Theoret. Phys. 59 (1) (1978) 55.

[34] S. Marculescu, et al., Nuclear Phys. B 349 (1991) 463.

[35] E. Gozzi, D. Mauro, A. Silvestri, Internat. J. Modern Phys. A 20 (2005) 5009. hep-th/0410129.

[36] P. Carta, E. Gozzi, D. Mauro, Ann. Phys. 15 (2006) 177. hep-th/0508244.

[37] A.F. Neto, et al. Diagrammar in Classical YM Theory (work in progress).

[38] E. Cattaruzza, Diagrammar in Classical Scalar Field Theory II (work in progress).

[39] C.D. Palmer, M.E. Carrington, Can. J. Phys. 80 (2002) 847-852. 